\title{
Lipid availability determines fate of skeletal progenitor cells via SOX9
}

Citation for published version (APA):

van Gastel, N., Stegen, S., Eelen, G., Schoors, S., Carlier, A., Daniels, V. W., Baryawno, N., Przybylski, D., Depypere, M., Stiers, P-J., Lambrechts, D., Van Looveren, R., Torrekens, S., Sharda, A., Agostinis, P., Lambrechts, D., Maes, F., Swinnen, J., Geris, L., ... Carmeliet, G. (2020). Lipid availability determines fate of skeletal progenitor cells via SOX9. Nature, 579(7797), 111-117. https://doi.org/10.1038/s41586-0202050-1

Document status and date:

Published: 05/03/2020

DOI:

10.1038/s41586-020-2050-1

Document Version:

Publisher's PDF, also known as Version of record

Document license:

Taverne

Please check the document version of this publication:

- A submitted manuscript is the version of the article upon submission and before peer-review. There can be important differences between the submitted version and the official published version of record.

People interested in the research are advised to contact the author for the final version of the publication, or visit the DOI to the publisher's website.

- The final author version and the galley proof are versions of the publication after peer review.

- The final published version features the final layout of the paper including the volume, issue and page numbers.

Link to publication

\footnotetext{
General rights rights.

- You may freely distribute the URL identifying the publication in the public portal. please follow below link for the End User Agreement:

www.umlib.nl/taverne-license

Take down policy

If you believe that this document breaches copyright please contact us at:

repository@maastrichtuniversity.nl

providing details and we will investigate your claim.
}

Copyright and moral rights for the publications made accessible in the public portal are retained by the authors and/or other copyright owners and it is a condition of accessing publications that users recognise and abide by the legal requirements associated with these

- Users may download and print one copy of any publication from the public portal for the purpose of private study or research.

- You may not further distribute the material or use it for any profit-making activity or commercial gain

If the publication is distributed under the terms of Article $25 \mathrm{fa}$ of the Dutch Copyright Act, indicated by the "Taverne" license above, 


\section{Lipid availability determines fate of skeletal progenitor cells via SOX9}

https://doi.org/10.1038/s41586-020-2050-1

Received: 1 July 2016

Accepted: 8 January 2020

Published online: 26 February 2020

Check for updates

\author{
Nick van Gastel ${ }^{1,2,3,4,5}$, Steve Stegen ${ }^{1,2}$, Guy Eelen ${ }^{6,7}$, Sandra Schoors $^{6,7}$, Aurélie Carlier ${ }^{2,8,9,10}$, \\ Veerle W. Daniëls ${ }^{11,12}$, Ninib Baryawno ${ }^{3,4,5,13}$, Dariusz Przybylski ${ }^{14}$, Maarten Depypere ${ }^{15,16}$, \\ Pieter-Jan Stiers ${ }^{1,2}$, Dennis Lambrechts ${ }^{2,17,18}$, Riet Van Looveren', Sophie Torrekens', \\ Azeem Sharda ${ }^{3,4,5}$, Patrizia Agostinis ${ }^{19}$, Diether Lambrechts ${ }^{20,21}$, Frederik Maes ${ }^{15,16}$, \\ Johan V. Swinnen" , Liesbet Geris ${ }^{2,8,9}$, Hans Van Oosterwyck ${ }^{2,8}$, Bernard Thienpont ${ }^{20,21}$, \\ Peter Carmeliet ${ }^{6,7}$, David T. Scadden ${ }^{3,4,5}$ \& Geert Carmeliet ${ }^{1,2 \bowtie}$
}

\begin{abstract}
The avascular nature of cartilage makes it a unique tissue $\mathrm{e}^{1-4}$, but whether and how the absence of nutrient supply regulates chondrogenesis remain unknown. Here we show that obstruction of vascular invasion during bone healing favours chondrogenic over osteogenic differentiation of skeletal progenitor cells. Unexpectedly, this process is driven by a decreased availability of extracellular lipids. When lipids are scarce, skeletal progenitors activate forkhead box O (FOXO) transcription factors, which bind to the Sox 9 promoter and increase its expression. Besides initiating chondrogenesis, SOX9 acts as a regulator of cellular metabolism by suppressing oxidation of fatty acids, and thus adapts the cells to an avascular life. Our results define lipid scarcity as an important determinant of chondrogenic commitment, reveal a role for FOXO transcription factors during lipid starvation, and identify SOX9 as a critical metabolic mediator. These data highlight the importance of the nutritional microenvironment in the specification of skeletal cell fate.
\end{abstract}

Bone repair reiterates the developmental endochondral ossification process and is initiated by periosteal skeletal progenitor cells that first form an avascular cartilage template which is later replaced by bone $\mathrm{e}^{1,2}$. Among the factors involved in chondrogenesis, the transcription factor SOX9 has been the most extensively studied, but how it is induced in skeletal progenitor cells is poorly understood. Since cartilage is avascular, the absence of blood vessels itself has been suggested to initiate chondrogenesis ${ }^{3-6}$, but a causal link has not been confirmed and remains controversial ${ }^{7}$. In this study, we provide evidence that local blood vessel availability determines skeletal progenitor cell fate during bone healing through a multifaceted mechanism involving lipid metabolism, FOXO signalling and SOX9.

\section{Vascularity controls skeletal cell fate}

To investigate whether the absence of vasculature determines skeletal progenitor fate we transplanted viable (autologous) bone grafts into femoral defects in mice, inducing a periosteal-driven healing response ${ }^{8}$. Periosteal progenitor cells near the host-graft border formed cartilage, whereas cells in the centre differentiated directly into bone-forming osteoblasts (Extended Data Fig. 1a). Periosteal cells did not contribute to blood vessels in the callus (Fig. 1a), but actively promoted vascular ingrowth as their removal reduced bone formation and callus vascularization (Extended Data Fig. 1b-d). At post-fracture day (PFD) 7 the central periosteal callus vasculature was highly connected with that of the surrounding muscle (Fig. 1b), suggesting that periosteal cells attract blood vessels from this site. To investigate the importance of this vascular ingrowth for bone repair, we inserted polycarbonate filters with different pore sizes between graft and muscle (Fig. 1c). Inserting a 30- $\mu \mathrm{m}$-pore filter still allowed capillaries to transverse the pores at PFD7, whereas a $0.2-\mu \mathrm{m}$-pore size prevented vascular ingrowth into the periosteal layer, as shown by the numerous capillaries adjacent to the filter on the muscle side and reduced callus vascularization (Fig. 1d). Concomitantly, periosteal cellularity decreased because of reduced proliferation and moderately increased cell death (Extended Data Fig. 2a, b), but more importantly, 


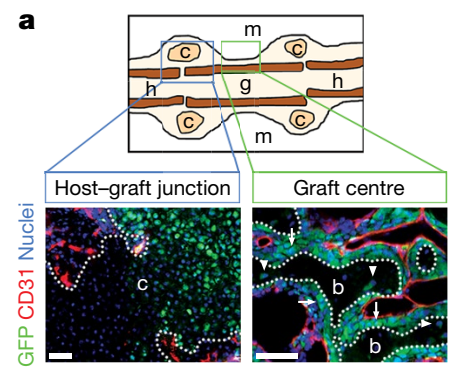

d

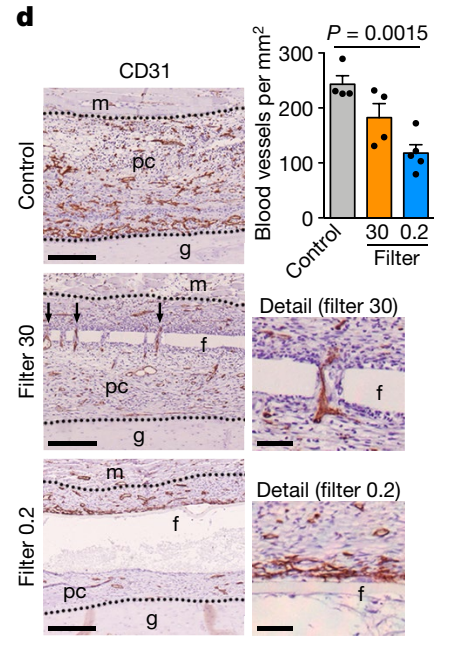

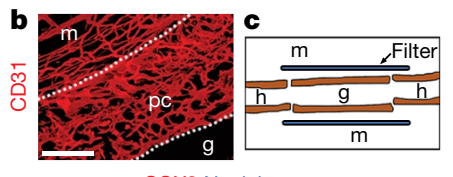
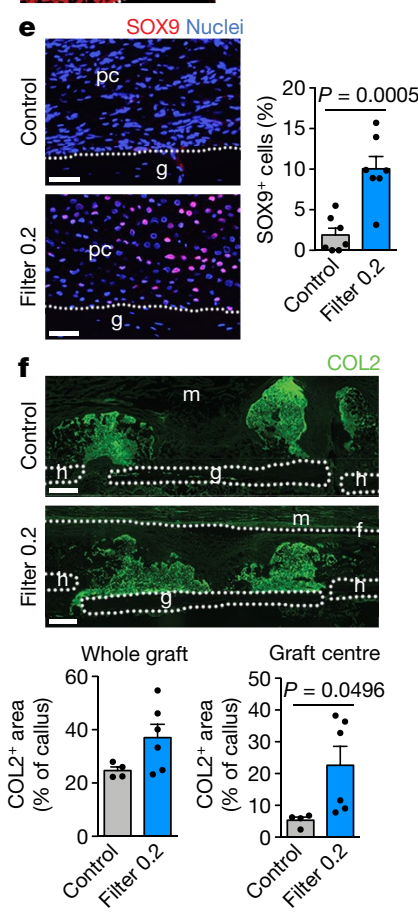

Fig. 1 Preventing vascular ingrowth during bone healing induces chondrogenesis. a, Immunofluorescence analysis of bone-graft periosteal cell tracing showing contribution to cartilage and bone (arrows, $\mathrm{GFP}^{+}$osteoblasts; arrowheads, $\mathrm{GFP}^{+}$osteocytes) in the graft callus at PFD14, while CD31 ${ }^{+}$blood vessels (red) are mainly host-derived (representative images of 4 mice). Scale bars, $50 \mu \mathrm{m}$. b, Immunofluorescence analysis of a bone-autograft section revealing the interconnected periosteal callus and skeletal muscle vasculature at PFD7 (representative image of 3 mice). Scale bar, $200 \mu \mathrm{m}$. c, Schematic representation of the autograft model with filter. d, Immunohistochemical analysis and quantification of callus vascularization at PFD7 when a filter with $30 \mu \mathrm{m}$ (filter 30; arrows indicate blood vessels passing through filter pores) or $0.2 \mu \mathrm{m}$ (filter 0.2) pore size was placed in between muscle and graft $(n=4$ mice for control and filter $30, n=5$ mice for filter 0.2 ). Scale bars, $50 \mu \mathrm{m}$ in detail images, otherwise $200 \mu \mathrm{m}$.e, Visualization and quantification of early chondrogenic cells in the callus of grafts with or without a filter $(0.2 \mu \mathrm{m})$ at PFD7 by immunofluorescence for SOX9 $(n=7$ mice). Scale bars, $50 \mu \mathrm{m}$.

f, Visualization and quantification of cartilage in the callus of autografts with and without filter $(0.2 \mu \mathrm{m})$ at PFD14 by immunofluorescence for collagen type 2 (COL2) ( $n=4$ mice for control, $n=6$ mice for filter 0.2$)$. Scale bars, $500 \mu \mathrm{m}$.

b, bone; c, cartilage; f, filter; g, graft; h, host; $m$, muscle; pc, periosteal callus. Data are mean \pm s.e.m.; one-way ANOVA with Bonferroni post hoc test (d), twotailed Student's $t$-test $(\mathbf{e}, \mathbf{f})$.

the number of $\mathrm{SOX} 9^{+}$early chondrogenic cells was higher at the central graft region (Fig. 1e). This chondrogenic switch resulted in less bone (Extended Data Fig. $2 \mathrm{c}$ ) but more type 2 collagen $(\mathrm{COL} 2)^{+}$cartilage matrix in the central region at PFD14 (Fig. 1f), where graft cells differentiated to chondrocytes instead of osteoblasts (Extended Data Fig. 2d). At PFD28, successful healing was observed in both conditions, although the presence of small cartilage islands in the callus with filter ( $75.0 \pm 14.4 \%$ of sections) suggests delayed healing (Extended Data Fig. 2e,f). Thus, skeletal progenitor cells undergo chondrogenic rather than osteogenic differentiation when blood supply is limited, securing successful bone healing.

During bone healing, the vasculature supplies nutrients (oxygen, glucose, amino acids and lipids), growth factors and perivascular

progenitor cells ${ }^{9}$. To distinguish between these components, we applied a computational model of bone healing ${ }^{10,11}$ to our bonegraft setup, in which cell fate and tissue formation are controlled by nutrient availability, osteochondrogenic growth factors, matrix density and local cell number (Extended Data Fig. 3a, b). The model correctly described the spatiotemporal progression of normal bonegraft healing (that is, blood vessels can come from the muscle; compare Extended Data Fig. 3b with Extended Data Fig. 1a). When the presence of a filter was mimicked by limiting diffusion of nutrients from the muscle side (20-40\% of the nutrients normally supplied by the vasculature), the model recapitulated the chondrogenic switch in the central graft region (Extended Data Fig. 3c, d). An additional supply of growth factors and/or progenitor cells from the muscle side did not significantly affect this bone repair profile (Extended Data Fig. 3e). The in silico model thus supports the hypothesis that nutrients supplied by the vasculature regulate skeletal progenitor cell differentiation.

\section{Lipid scarcity induces chondrogenesis}

To test this hypothesis, we investigated the nutritional control of cell fate using two models of skeletal progenitors: the $\mathrm{C} 3 \mathrm{H} 10 \mathrm{~T} 1 / 2$ cell line, a homogeneous population retaining multipotency properties ${ }^{12}$, and primary murine periosteal cells, which are more heterogeneous but contain true skeletal stem and progenitor cells ${ }^{13-15}$. We confirmed key findings in immunophenotypically-defined skeletal stem cells isolated from total long bones of newborn mice ${ }^{16}$, which are homogeneous but limited in number.

Combined nutrient deprivation (CND; reduced levels of serum, oxygen, glucose and glutamine) increased SOX9 protein and mRNA levels in $\mathrm{C} 3 \mathrm{H} 10 \mathrm{~T} 1 / 2$ or periosteal cells, without changes in expression of osteogenic, adipogenic or myogenic transcription factors (Fig. 2a, Extended Data Fig. $4 \mathrm{a}-\mathrm{c}$ ). Depriving C3H10T1/2 cells of individual nutrients revealed that low oxygen levels increased SOX9, as reported ${ }^{17,18}$, whereas lowering glucose or glutamine levels had little effect (Fig. 2b). Unexpectedly, serum deprivation led to massive and rapid accumulation of SOX9 mRNA and protein, resulting from increased transcription and translation (Fig. 2b, Extended Data Fig. 4d-g). Expression of osteogenic, adipogenic and myogenic transcription factors did not change (Extended Data Fig. 4h). Serum deprivation also increased SOX9 in periosteal cells (Extended Data Fig. 4i) and enhanced their chondrogenic differentiation in micromass cultures (Fig. 2c), but prevented osteogenic differentiation (Extended Data Fig. 4j). A possible explanation for this chondrogenic switch is avoiding cell death. Indeed, knockdown of SOX9 in C3H10T1/2 cells, periosteal cells and growth plate-derived chondrocytes reduced cell viability in CND, and to a minor extent also in serum deprivation (Extended Data Fig. $4 \mathrm{k}, \mathrm{l}$ ). Thus, skeletal progenitor cells rapidly adapt to specific nutritional stress by increasing SOX9 levels and undergoing chondrogenic commitment.

Serum represents the main source of lipids, and we questioned whether serum deprivation-induced chondrogenic commitment of skeletal progenitors could be attributed to lipid deprivation. Resupplying C3H10T1/2 cells with oleate (Fig. 2d), palmitate, very low density lipoproteins or polyunsaturated fatty acids (PUFA) (Extended Data Fig. $5 \mathrm{a}-\mathrm{c}$ ) prevented the increase in SOX 9 during serum deprivation. In addition, lipid-reduced serum (LRS) mimicked the effects of serum deprivation. LRS increased SOX9 levels in C3H10T1/2 cells (Fig. 2e), promoted chondrogenic differentiation of periosteal cells in micromass or pellet cultures, an effect partially reversed by exogenous fatty acids (Fig. 2f, Extended Data Fig. 5d), and inhibited their osteogenic differentiation (Extended Data Fig. 5e). Importantly, serum deprivation or LRS also increased SOX9 levels in skeletal stem cells (Extended Data Fig. 5f). In all studied cell types, lipid deprivation increased the number of SOX $9^{\text {high }}$ cells, and cell cycle and apoptosis analysis showed this was 

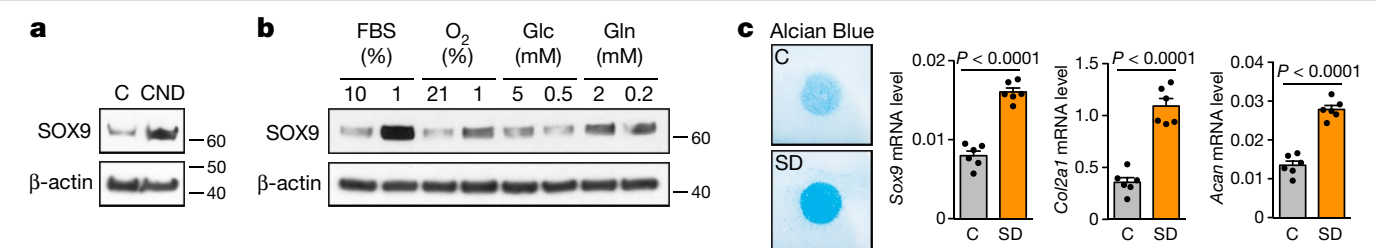

d

$\mathrm{SD}+$ oleate

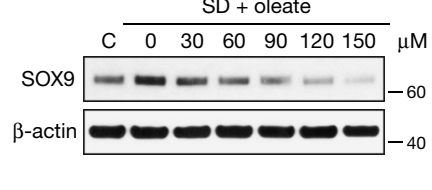

e
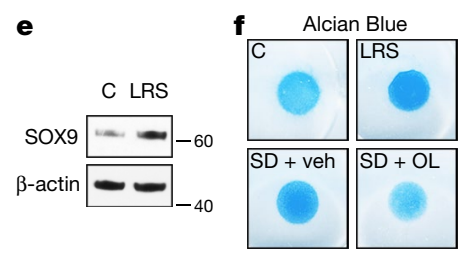
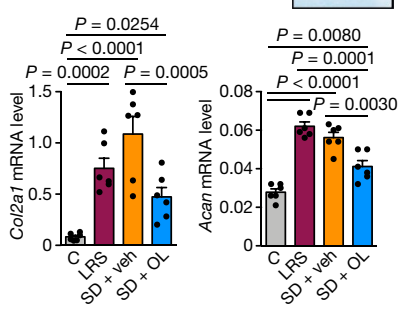

g

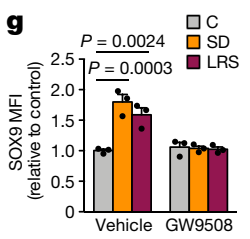

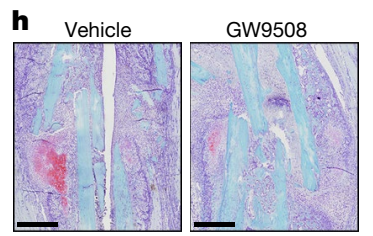
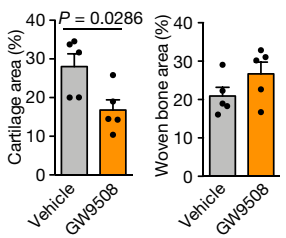

Fig. 2 | Lipid scarcity induces SOX9 in skeletal progenitors. a, b, Immunoblot detection of total SOX9 in C3H10T1/2 cells exposed for $24 \mathrm{~h}$ to control (C) or CND medium (a) or to different nutritional stresses (FBS, fetal bovine serum; Glc, glucose; Gln, glutamine) (b), with $\beta$-actin as loading control $(n=2$ independent experiments).c, Chondrogenic differentiation of periosteal cells in control or serum deprivation (SD) medium, assessed by visualization of chondrogenic matrix deposition (alcian blue staining) and quantification of Sox 9, Col2a1 and Acan mRNA levels (relative to $A c t b, n=6$ biologically independent samples). d, e, Immunoblot detection of total SOX9 in C3H10T1/2 cells exposed for $6 \mathrm{~h}$ to control medium, SD medium, SD medium supplemented with increasing concentrations of oleate (d) or LRS medium (e), with $\beta$-actin as loading control ( $n=2$ independent experiments).

f, Chondrogenic differentiation of periosteal cells in control medium, LRS

not due to selection of a pre-existing SOX $9^{\text {high }}$ population (Extended Data Fig. $5 \mathrm{f}-\mathrm{h})$.

We next tested whether lipid availability also controls skeletal progenitor differentiation in more physiologically relevant settings. Since it is not feasible to locally deprive cells specifically of exogenous lipids in vivo, we first used embryonic metatarsal cultures, an organ-like ex vivo model of bone development. Serum deprivation increased the number of $\mathrm{SOX}^{+}$chondrocytes and prevented osteogenesis, evidenced by absence of Col1a1-expressing cells and mineralization, which was reversed by fatty acid supplementation (Extended Data Fig. $5 i, j$ ). Second, local injection of fatty acids during fracture repair reduced the amount of cartilage in the callus, with no change in newly formed bone (Extended Data Fig. 5k). Third, GW9508, an agonist of free fatty acid receptor 1 (FFAR1) and FFAR4, prevented the increase in SOX 9 induced by serum deprivation or LRS in the three cell models (Fig. 2g; Extended Data Fig. 5l). Accordingly, locally injecting GW9508 during fracture repair decreased cartilage in the callus without affecting woven bone areas (Fig. 2h). Together, low local lipid levels promote chondrogenesis of skeletal progenitor cells in vivo.

Our findings suggest that the chondrogenic switch during bonegraft healing in the presence of a filter (Fig. 1) is primarily due to the absence of exogenous lipids, which is linked to poor vascularization. We found that diffusion of lipids in a collagen gel containing periosteal cells is much lower than that of glucose (Extended Data Fig. $5 \mathrm{~m}$ ), indicating that lipids are a limiting nutrient when vascularization is inadequate. Furthermore, we showed that the absence of specific cell types, potentially blocked by the filter, does not impact chondrogenesis. Indeed, serum deprivation-supported chondrogenic differentiation of periosteal cells in micromass cultures was not prevented by muscle-derived endothelial cells, macrophages or pericytes, in contrast to fatty acid supplementation. (Extended Data Fig. 5n, o). Together with our in vivo (Fig. 1) and in silico (Extended Data Fig. 3) results, this shows that lipid deprivation caused by reduced vascularization is probably an important determinant of periosteal chondrogenesis during bone healing. medium, SD medium or SD medium supplemented with $60 \mu \mathrm{M}$ oleate $(\mathrm{OL})$, assessed by alcian blue staining and quantification of Col2a1 and Acan mRNA levels (relative to $A c t b, n=6$ biologically independent samples). Veh, vehicle. g, Flow cytometric quantification of total SOX9 levels in periosteal cells exposed for 24 h to control medium, SD medium or LRS medium supplemented with $100 \mu \mathrm{M} \mathrm{GW} 9508$ (FFAR1/4 agonist) or vehicle (DMSO) $(n=3$ biologically independent samples). h, Histological visualization (safranin O staining) and quantification of cartilage and woven bone in the callus at PFD7 of mice treated daily with GW9508 (10 nmol) or vehicle (0.2\% DMSO in saline) at the fracture site ( $n=5$ mice). Scale bars, $500 \mu \mathrm{m}$. Data are mean \pm s.e.m.; two-tailed Student's $t$-test (c, h), one-way ANOVA (f) or two-way ANOVA (g) with Bonferroni post hoc test. For gel source data, see Supplementary Fig. 1.

\section{Chondrocytes have low fatty acid oxidation}

Why would chondrogenic commitment be beneficial when lipids are scarce? We hypothesized that chondrocyte metabolism does not rely on exogenous lipids. To test this, we compared the metabolic profile of chondrocytes to that of skeletal progenitors and mature osteoblasts (Fig. 3a, Extended Data Fig. 6a). Chondrocytes were highly glycolytic, as reported ${ }^{19,20}$. Osteoblasts showed the highest oxygen consumption rate (OCR), which was not owing to high glucose oxidation, but to a higher rate of fatty acid oxidation (FAO). Chondrocytes exhibited low FAO and skeletal progenitors had an intermediate profile. To confirm these findings in vivo, we examined metabolic-gene signatures in a mouse long-bone single-cell RNA-sequencing (RNA-seq) dataset that we generated recently ${ }^{21}$. This atlas encompasses 17 non-haematopoietic cell types including skeletal progenitors, chondrocytes and osteoblasts (Extended Data Fig. 6b). The different chondrocyte populations (clusters 2,10,13,17; Sox $^{+}$Acan $^{+}$) showed low expression of FAO genes and high expression of glycolytic genes compared with osteoblasts (clusters 7 and 8; $\mathrm{Colla1}^{+} \mathrm{Ocn}^{+}$; Ocn is also known as Bglap) and, to a minor extent, skeletal progenitors (clusters 1 and 4 ; Grem1 $1^{+}$) (Extended Data Fig. 6b, c). Gene expression analysis confirmed higher expression of the glycolytic genes Slc2a1 (encoding GLUT1), Pfkfb3 and $L d h a$, but lower expression of the FAO-related genes Cpt1a, Acadm and Acadl in growth plate cartilage versus cortical bone samples (Extended Data Fig. 6d). Immunohistochemistry showed low CPT1a levels and high GLUT1 levels in chondrocytes of the growth plate and fracture callus, whereas trabecular bone osteoblasts displayed high levels of both CPT1a and GLUT1 (Fig. 3b). Intravenous injection of fluorescent fatty acid and glucose analogues revealed labelled fatty acids in osteoblasts but not in chondrocytes in the growth plate or fracture callus, whereas labelled glucose was taken up by both cell types (Extended Data Fig. 6e, f), confirming that low FAO in chondrocytes correlates with lipid scarcity. Transplantation experiments showed that loss of CPT1a abrogates osteogenic differentiation of skeletal stem cells during fracture healing but preserves their ability to become chondrocytes (Fig.3c, Extended Data Fig. 6g). In addition, etomoxir, a CPT1 inhibitor, decreased viability 

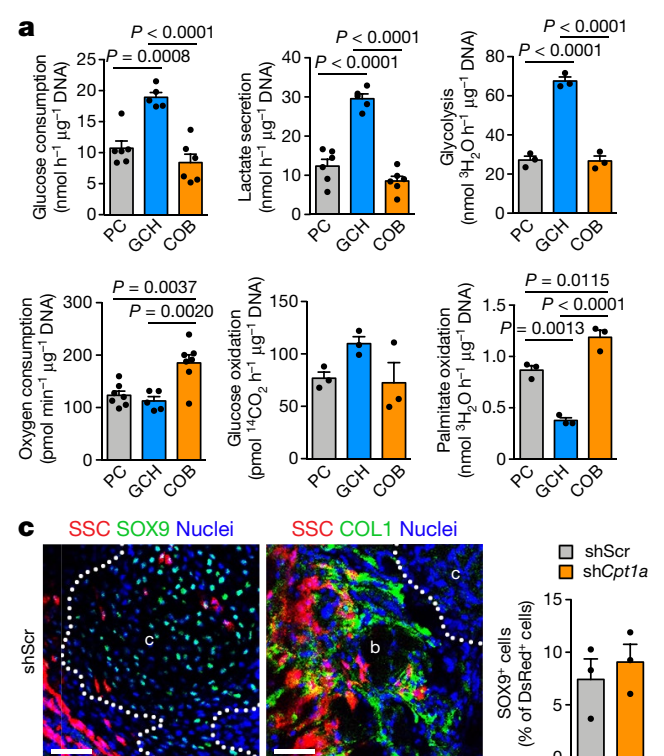

b

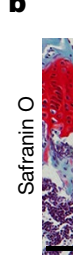
Growth plate Fracture callus
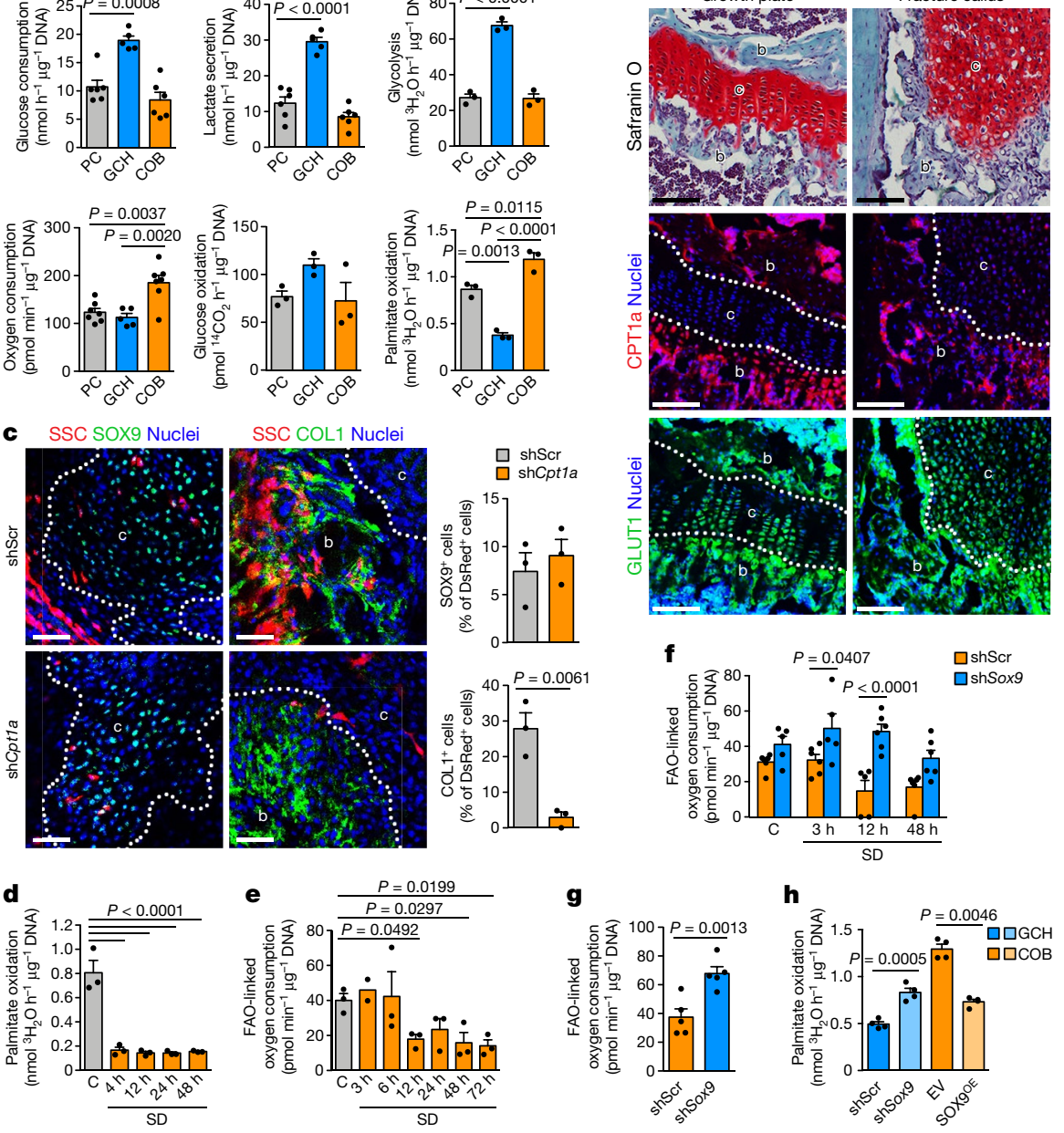

Fig. 3 |SOX9 suppresses FAO in chondrocytes. a, Quantification of glucose consumption and lactate secretion (PC, $\mathrm{COB}: n=6 ; \mathrm{GCH}: n=5$ biologically independent samples), glycolytic rate ( $n=3$ biologically independent samples), oxygen consumption (PC, $\mathrm{COB}: n=7 ; \mathrm{GCH}: n=5$ biologically independent samples), glucose oxidation ( $n=3$ biologically independent samples) and palmitate oxidation ( $n=3$ biologically independent samples) in periosteal cells $(\mathrm{PC})$, growth plate-derived chondrocytes $(\mathrm{GCH})$ and calvarial osteoblasts (COB).b, Analysis of adjacent histological sections of a growth plate and fracture callus (PFD7) by safranin O staining (cartilage) or immunofluorescence for CPT1a or GLUT1 (representative images of 3 mice). Scale bars, $100 \mu \mathrm{m}$. Dotted white lines delineate cartilage areas. c, Histological visualization and quantification of early chondrogenic ( $\mathrm{SOX} 9^{+}$) and osteogenic $\left(\mathrm{COL1}^{+}\right)$cells in the callus of fractures (PFD7) transplanted with CAG-DsRed ${ }^{+}$ skeletal stem cells (SSC) transduced with shRNA against $C p t 1 a$ (shCpt1a) or scrambled shRNA control (shScr) $(n=3$ mice). Scale bars, $50 \mu \mathrm{m}$.

and numbers of cultured calvarial osteoblasts but not growth platederived chondrocytes (Extended Data Fig. 6h). Thus, chondrocytes exhibit a low rate of FAO consistent with local lipid scarcity, and do not depend on this pathway to fulfil their metabolic demands.

\section{SOX9 suppresses FAO}

We next determined how lipid deprivation affects the rate of FAO in skeletal progenitor cells. As expected, oxidation of extracellular palmitate immediately dropped after exposing periosteal cells to serum deprivation or LRS (Fig. 3d, Extended Data Fig. 7a). Surprisingly, cells temporarily maintained total $\mathrm{FAO}$, which was quantified indirectly by measuring etomoxir-sensitive $\mathrm{OCR}^{22}$, for $6 \mathrm{~h}$ after serum deprivation (Fig. 3e, Extended Data Fig. 7b), suggesting that they initially

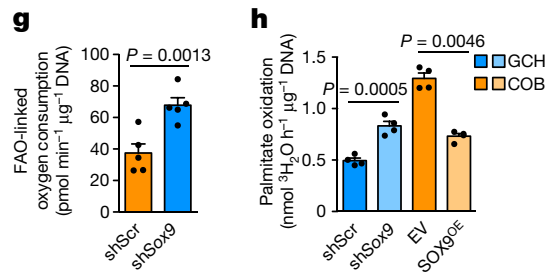

d, Measurement of oxidation of extracellularly added palmitate by periosteal cells in control medium or at different times in SD medium ( $n=3$ biologically independent samples).e, Quantification of FAO-linked OCR in periosteal cells in control medium or at different times in SD medium ( $3 \mathrm{~h}: n=2$, other time points: $n=3$ biologically independent samples).f, Quantification of FAO-linked OCR in periosteal cells transduced with shSox 9 or shScr in control medium or at different times in SD medium (shScr $12 \mathrm{~h}$, shSox 9 control, shSox $93 \mathrm{~h}: n=5$; all others: $n=6$ biologically independent samples). $g$, Quantification of FAOlinked OCR in GCH transduced with shSox 9 or $\operatorname{shScr}(n=5$ biologically independent samples). $\mathbf{h}$, Quantification of palmitate oxidation in $\mathrm{GCH}$ transduced with shSox 9 or shScr, and in $\mathrm{COB}$ transduced with a lentiviral vector encoding SOX9 (SOX9 ${ }^{\mathrm{OE}}$, SOX9 overexpression) or an empty vector (EV) $(n=4$ biologically independent samples). Data are mean \pm s.e.m.; one-way ANOVA (a, d, e) or two-way ANOVA (f) with Bonferroni post hoc test, two-tailed Student's $t$-test $(\mathbf{c}, \mathbf{g}, \mathbf{h})$.

compensate for the scarcity of exogenous lipids, possibly through mobilization of intracellular lipid stores. Indeed, fluorescent fatty acids translocated from lipid droplets into mitochondria, where FAO takes place, when periosteal cells were exposed to serum deprivation (Extended Data Fig. 7c). Starvation-induced lipid-droplet generation and breakdown are both linked to autophagy ${ }^{23,24}$, and we confirmed that $\mathrm{C} 3 \mathrm{H} 10 \mathrm{~T} 1 / 2$ cells and periosteal cells activate autophagy early after serum deprivation (Extended Data Fig. 7d-f). Accordingly, lipid-droplet number initially increased during serum deprivation in C3H1OT1/2 cells before decreasing at $6 \mathrm{~h}$, and knockdown of the essential autophagosome protein ATG $5^{25}$ prevented both the initial increase and the late breakdown of lipid droplets after serum deprivation (Extended Data Fig. 7g). Furthermore, the lysosomotropic drug chloroquine immediately reduced the FAO-linked OCR upon exposure 

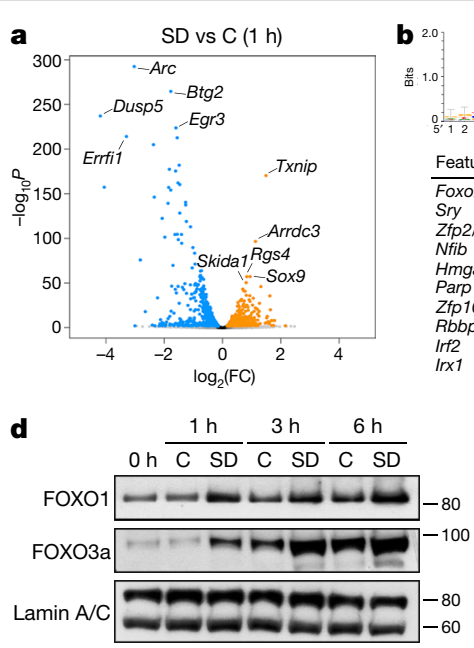

d
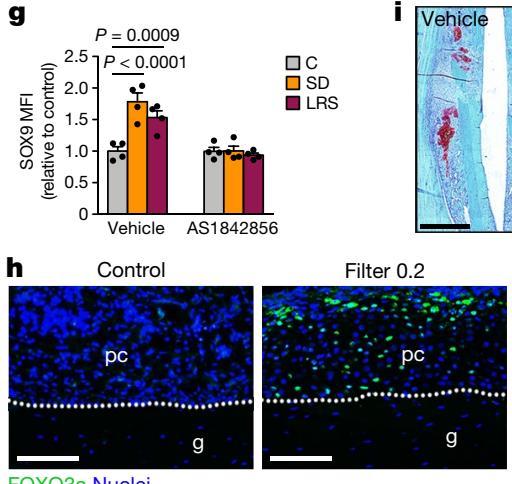

Fig. 4 | Lipids regulate SOX9 through FOXO signalling. a, b, Volcano plot showing significantly enriched and depleted mRNAs (a) and top-10 mostsignificantly enriched transcription factor motifs with normalized enrichment scores (NES) as determined by $\mathrm{i}$-cisTarget analysis (b) in C3H10T1/2 cells exposed for $1 \mathrm{~h}$ to SD medium versus control medium ( $n=3$ replicates). Motif shown on top is the FOXO/forkhead motif.c, Confocal microscopy of C3H10T1/2 cells stained for FOXO1 (top) or FOXO3a (bottom) shows increased nuclear localization after exposure of cells for $3 \mathrm{~h}$ to SD medium or LRS medium (representative images of 2 independent experiments). Scale bars, $20 \mu \mathrm{m}$. d, Immunoblot detection of nuclear FOXO1 and FOXO3a in C3H10T1/2 cells exposed for 1,3 or $6 \mathrm{~h}$ to control medium or SD medium, with lamin $\mathrm{A} / \mathrm{C}$ as loading control ( $n=2$ independent experiments). e, Nuclear FOXO activity in C3H10T1/2 cells exposed for $3 \mathrm{~h}$ to control medium, SD medium or LRS medium supplemented with vehicle (EtOH), oleate $(60 \mu \mathrm{M})$ or PUFA ( $n=3$ independent experiments).f, Occupancy of FOXO3a at the Sox 9 promoter of Cas9expressing C3H10T1/2 cells transduced with inducible short guide RNA (sgRNA) against FoxO1 (sgFoxo1), sgFoxo3a or a scrambled sgRNA (sgScr), exposed for $3 \mathrm{~h}$ to control medium or SD medium in the presence of doxycycline $\left(250 \mathrm{ng} \mathrm{ml}^{-1}\right)$, as determined by chromatin immunoprecipitation with quantitative PCR (ChIP-qPCR) ( $n=3$ independent experiments). g, Flow cytometric quantification of total SOX9 levels in periosteal cells exposed for

of periosteal cells to serum deprivation (Extended Data Fig. 7h) and decreased survival of $\mathrm{C} 3 \mathrm{H} 10 \mathrm{~T} 1 / 2$ cells and periosteal cells during serum deprivation (Extended Data Fig. 7i). Together, these data show that skeletal progenitors depend on lysosome-mediated mobilization of intracellular lipid stores to temporarily support FAO and secure survival when extracellular lipids become limited.

The increase in SOX9 levels (Extended Data Fig. 4d, e) and the decrease in total FAO (Fig. 3e) occur concomitantly after lipid deprivation, suggesting that they are connected. Deletion of SOX9 in periosteal cells prevented the suppression of FAO by serum deprivation (Fig. 3f), whereas inhibition of FAO with etomoxir did not alter SOX9 levels (Extended Data Fig. 7j). Moreover, knockdown of SOX9 in

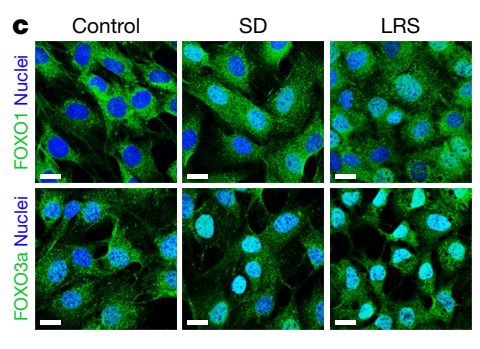

f $\quad \frac{P<0.0001}{P=0.0008}$

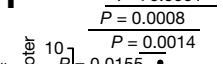

$\frac{P=0.0369}{P=0.0002}$

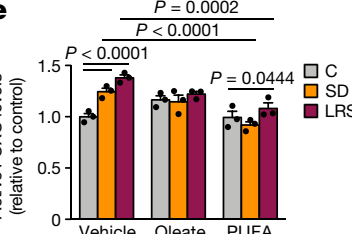

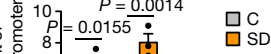
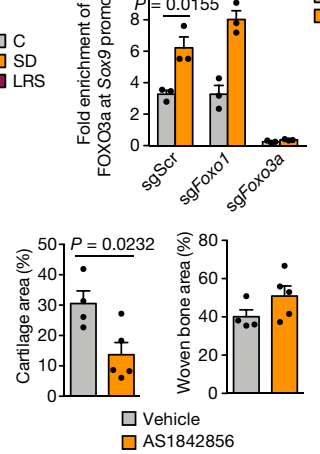

$\square$ AS1842856

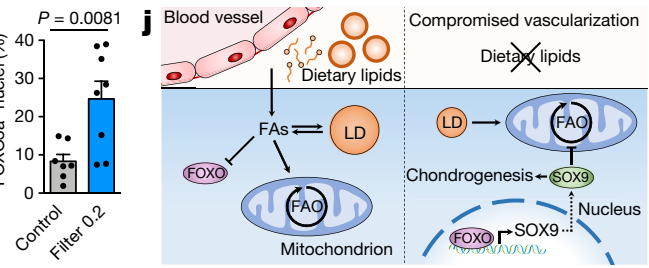

$24 \mathrm{~h}$ to control medium, SD medium or LRS medium supplemented with $1 \mu \mathrm{M}$ AS1842856 (FOXO inhibitor) or vehicle (DMSO) ( $n=4$ biologically independent samples). h, Histological visualization and quantification of FOXO3aexpressing cells in the central periosteal callus of grafts with or without a filter ( $0.2 \mu \mathrm{m}$ pore size) at PFD7 (control: $n=7$, filter $0.2: n=8$ mice). Scale bars, $50 \mu \mathrm{m}$. i, Histological visualization (safranin O staining) and quantification of cartilage and woven bone in the callus at PFD7 of mice treated daily with AS1842856 (500 pmol) or vehicle (0.1\% DMSO in saline) at the fracture site (vehicle: $n=4$, AS1842856: $n=5$ mice). Scale bars, $500 \mu \mathrm{m}$.j, Schematic overview of main findings. During bone fracture healing, skeletal progenitor cells in adequately vascularized environments differentiate into osteoblasts, which require high levels of exogenous fatty acids (FA) to fuel their FAOdependent metabolism. Cells in regions with a poor vascular supply will temporarily support FAO by breaking down intracellular lipid droplets (LD), while the lack of dietary lipids simultaneously increases FOXO activity. FOXOs increase levels of SOX9, which activates the chondrogenic program and blocks FAO. These adaptations promote cell survival and secure bone healing even in nutrient-poor environments. Data are mean \pm s.e.m.; two-way ANOVA with Bonferroni post hoc test $(\mathbf{e}-\mathbf{g})$, two-tailed Student's $t$-test $(\mathbf{h}, \mathbf{i})$. For gel source data, see Supplementary Fig. 1.

growth plate-derived chondrocytes induced not only loss of typical chondrocyte characteristics such as cobblestone-like morphology and expression of Col2a1 and Acan (Extended Data Fig. 7k, I), but also increased expression of Cpt1a and $A c a d l$ (Extended Data Fig. 7l) and the rate of FAO in chondrocytes (Fig. 3g, h). By contrast, overexpression of SOX9 in calvarial osteoblasts decreased FAO (Fig. 3h).SOX9 thus acts as a metabolic regulator in chondrogenic cells by suppressing FAO.

\section{FOXOs induce SOX9 upon lipid starvation}

We next examined how lipids regulate SOX9 levels. Transcriptomics showed robust upregulation of Sox 9 expression in C3H1OT1/2 cells 
starting $1 \mathrm{~h}$ after serum deprivation and increased expression of several other, but not all, chondrogenic markers from $3 \mathrm{~h}$ onwards (Extended Data Fig. 8a). Differential expression analysis showed that $678(1 \mathrm{~h})$, $4,022(3 \mathrm{~h})$ and $3,811(6 \mathrm{~h})$ genes were significantly upregulated by serum deprivation, including Sox 9 as one of the top hits at all time points (Fig. 4a, Extended Data Fig. 8b). A total of 757 (1 h), 2,167 (3 h) and 3,872 (6h) genes were significantly downregulated, including genes associated with proliferation (Egr3, Dusp5 and Errfi1), skeletal stem cells (Nes and Itga5) and osteogenesis (Spp1 and Adam19) (Fig. 4a; Extended Data Fig. 8b). Transcription factor-binding-motif analysis ${ }^{26}$ of the top-100 overexpressed genes at each time point showed strong enrichment of the FOXO/forkhead motif (Fig. 4b, Extended Data Fig. 8c). We confirmed that serum deprivation increases nuclear FOXO1 and FOXO3a in C3H1OT1/2 cells (Fig. 4c, d) and active FOXO levels in C3H1OT1/2 and skeletal stem cells, an effect prevented by exogenous fatty acids (Fig. 4e, Extended Data Fig. 8d-f), indicating that extracellular lipids control FOXO activity. More specifically, FOXO1 and FOXO3a showed increased binding to the Sox 9 promoter during serum deprivation (Fig. 4f, Extended Data Fig. 8g), and the FOXO inhibitor AS1842856 prevented induction of SOX 9 during lipid deprivation in all cell types (Fig. 4g, Extended Data Fig. 8h). Similar results were obtained using a CRISPR-Cas9 approach to conditionally delete Foxo1 and Foxo3a (also known as Foxo3) in C3H10T1/2 cells, or using short hairpin RNAs (shRNAs) in skeletal stem cells (Extended Data Fig. 8i,j). These data demonstrate that FOXOs directly control Sox 9 transcription during lipid deprivation.

We next confirmed the relation between lipid deprivation, FOXOs and SOX9 during bone healing. First, the presence of the filter $(0.2 \mu \mathrm{m})$ during bone-graft healing increased the number of cells positive for nuclear FOXO3a in the central periosteal region (Fig. 4h), similar to the increase in SOX $9^{+}$cells (Fig. 1e). Second, stimulation of fatty acid signalling using the FFAR1/4 agonist GW9508 during fracture healing strongly reduced the number of FOXO3a ${ }^{+}$nuclei in the periosteal callus (Extended Data Fig. 8k), correlating with reduced amounts of cartilage (Fig. $2 \mathrm{~h}$ ). Third, skeletal stem cells with FOXO1 and FOXO3 inactivation failed to engraft into tibial fractures (Extended Data Fig. 81), which may be owing to their inability to increase SOX9 levels upon lipid deprivation, or a general failure to survive transplantation-associated stress. Finally, local daily injection of the FOXO inhibitor AS1842856 during fracture healing reduced the amount of cartilage while not affecting new bone formation (Fig. 4i). Thus, FOXO signalling in vivo is negatively regulated by lipid availability and is required for skeletal progenitor cell chondrogenesis and survival during bone healing.

\section{Discussion}

On the basis of our findings, we propose a model in which the local vasculature, through supply of lipids, influences skeletal progenitor differentiation during fracture healing (Fig. 4j). Cells close to blood vessels become osteoblasts, which depend on FAO to support their metabolic demands. Skeletal progenitors in poorly vascularized regions sustain FAO for a short time by mobilizing intracellular lipid stores and then activate FOXO signalling as a result of exogenous lipid starvation. Nuclear localization of FOXOs promotes expression of SOX9, which induces chondrogenic commitment and suppresses FAO to allow longterm cell survival.

Low lipid levels are thus the main nutritional determinant for chondrogenic commitment of skeletal progenitor cells, rather than lack of oxygen or glucose $\mathrm{e}^{19,20,27}$, although growth factors are indispensable to activate the full chondrogenic-differentiation program $^{1,2,9}$. In contrast to osteoblasts ${ }^{28,29}$, we find that chondrocytes are largely independent of FAO, consistent with poor diffusion of fatty acids in cartilage tissue. This metabolic independence from extracellular lipids would therefore be beneficial in the avascular cartilage environment. FAO in chondrocytes is suppressed by SOX9, attributing a novel metabolic regulatory role to this transcription factor. Mechanistically, reduced lipid availability is translated into SOX9 production through FOXOs, well-known regulators of the cellular response to metabolic stress ${ }^{30}$. We propose lipid starvation as an additional trigger for FOXO activation, although the full signalling cascade and exact lipid sensor remain unknown. Of interest, osteoarthritis is associated with increased angiogenesis and $\mathrm{FAO}^{31,32}$ but reduced SOX9 levels and FOXO activity ${ }^{33,34}$. Our results show that all these phenomena may be connected to local lipid availability, suggesting that manipulation of lipid metabolism could be of therapeutic interest. More generally, our findings show that local nutrient levels can decide stem-cell lineage choice through direct transcriptional changes. As a consequence, the metabolic profile of a mature cell may reflect microenvironmental constraints as much as particular cellular needs.

\section{Online content}

Any methods, additional references, Nature Research reporting summaries, source data, extended data, supplementary information, acknowledgements, peer review information; details of author contributions and competing interests; and statements of data and code availability are available at https://doi.org/10.1038/s41586-020-2050-1.

1. Kronenberg, H. M. Developmental regulation of the growth plate. Nature 423, 332-336 (2003).

2. Roberts, S. J., van Gastel, N., Carmeliet, G. \& Luyten, F. P. Uncovering the periosteum for skeletal regeneration: the stem cell that lies beneath. Bone 70, 10-18 (2015).

3. Hallmann, R., Feinberg, R. N., Latker, C. H., Sasse, J. \& Risau, W. Regression of blood vessels precedes cartilage differentiation during chick limb development. Differentiation 34, 98-105 (1987).

4. Yin, M. \& Pacifici, M. Vascular regression is required for mesenchymal condensation and chondrogenesis in the developing limb. Dev. Dyn. 222, 522-533 (2001).

5. Maes, C. et al. Placental growth factor mediates mesenchymal cell development, cartilage turnover, and bone remodeling during fracture repair. J. Clin. Invest. 116, 12301242 (2006).

6. Taylor, D. K. et al. Thrombospondin-2 influences the proportion of cartilage and bone during fracture healing. J. Bone Miner. Res. 24, 1043-1054 (2009).

7. Miclau, K. R. et al. Stimulating fracture healing in ischemic environments: does oxygen direct stem cell fate during fracture healing? Front. Cell Dev. Biol. 5, 45 (2017).

8. Tiyapatanaputi, P. et al. A novel murine segmental femoral graft model. J. Orthop. Res. 22, 1254-1260 (2004).

9. Stegen, S., van Gastel, N. \& Carmeliet, G. Bringing new life to damaged bone: the importance of angiogenesis in bone repair and regeneration. Bone 70, 19-27 (2015).

10. Carlier, A. et al. MOSAIC: a multiscale model of osteogenesis and sprouting angiogenesis with lateral inhibition of endothelial cells. PLOS Comput. Biol. 8 , e1002724 (2012).

11. Carlier, A., Geris, L., van Gastel, N., Carmeliet, G. \& Van Oosterwyck, H. Oxygen as a critical determinant of bone fracture healing-a multiscale model. J. Theor. Biol. 365 247-264 (2015).

12. Zhao, L., Li, G., Chan, K. M., Wang, Y. \& Tang, P. F. Comparison of multipotent differentiation potentials of murine primary bone marrow stromal cells and mesenchymal stem cell line $\mathrm{C} 3 \mathrm{H} 10 \mathrm{~T} 1 / 2$. Calcif. Tissue Int. 84, 56-64 (2009).

13. van Gastel, N. et al. Engineering vascularized bone: osteogenic and proangiogenic potential of murine periosteal cells. Stem Cells 30, 2460-2471 (2012).

14. Debnath, S. et al. Discovery of a periosteal stem cell mediating intramembranous bone formation. Nature 562, 133-139 (2018).

15. Duchamp de Lageneste, $\mathrm{O}$. et al. Periosteum contains skeletal stem cells with high bone regenerative potential controlled by Periostin. Nat. Commun. 9, 773 (2018).

16. Chan, C. K. et al. Identification and specification of the mouse skeletal stem cell. Cell 160, 285-298 (2015)

17. Amarilio, R. et al. HIF1a regulation of Sox9 is necessary to maintain differentiation of hypoxic prechondrogenic cells during early skeletogenesis. Development 134, 39173928 (2007).

18. Robins, J. C. et al. Hypoxia induces chondrocyte-specific gene expression in mesenchymal cells in association with transcriptional activation of Sox9. Bone 37, 313-322 (2005).

19. Shapiro, I. M. \& Srinivas, V. Metabolic consideration of epiphyseal growth: survival responses in a taxing environment. Bone 40, 561-567 (2007).

20. Stegen, S. et al. HIF-1a metabolically controls collagen synthesis and modification in chondrocytes. Nature 565, 511-515 (2019).

21. Baryawno, N. et al. A cellular taxonomy of the bone marrow stroma in homeostasis and leukemia. Cell 177, 1915-1932.e16 (2019).

22. Kim, C. et al. Studying arrhythmogenic right ventricular dysplasia with patient-specific iPSCs. Nature 494, 105-110 (2013).

23. Singh, R. et al. Autophagy regulates lipid metabolism. Nature 458, 1131-1135 (2009).

24. Rambold, A. S., Cohen, S. \& Lippincott-Schwartz, J. Fatty acid trafficking in starved cells: regulation by lipid droplet lipolysis, autophagy, and mitochondrial fusion dynamics. Dev. Cell 32, 678-692 (2015). 
25. Tsukamoto, S. et al. Autophagy is essential for preimplantation development of mouse embryos. Science 321, 117-120 (2008).

26. Imrichová, H., Hulselmans, G., Atak, Z. K., Potier, D. \& Aerts, S. i-cisTarget 2015 update: generalized cis-regulatory enrichment analysis in human, mouse and fly. Nucleic Acids Res. 43 (W1), W57-W64 (2015).

27. Shang, J., Liu, H., Li, J. \& Zhou, Y. Roles of hypoxia during the chondrogenic differentiation of mesenchymal stem cells. Curr. Stem Cell Res. Ther. 9, 141-147 (2014).

28. Frey, J. L. et al. Wnt-Lrp5 signaling regulates fatty acid metabolism in the osteoblast. Mol. Cell. Biol. 35, 1979-1991 (2015).

29. Kim, S. P. et al. Fatty acid oxidation by the osteoblast is required for normal bone acquisition in a sex- and diet-dependent manner. JCl Insight 2, 92704 (2017).

30. Eijkelenboom, A. \& Burgering, B. M. FOXOs: signalling integrators for homeostasis maintenance. Nat. Rev. Mol. Cell Biol. 14, 83-97 (2013).
31. Ashraf, S. \& Walsh, D. A. Angiogenesis in osteoarthritis. Curr. Opin. Rheumatol. 20, 573580 (2008).

32. Ratneswaran, A. et al. Peroxisome proliferator-activated receptor $\delta$ promotes the progression of posttraumatic osteoarthritis in a mouse model. Arthritis Rheumatol. 67, 454-464 (2015).

33. Zhong, L., Huang, X., Karperien, M. \& Post, J. N. Correlation between gene expression and osteoarthritis progression in human. Int. J. Mol. Sci. 17, E1126 (2016).

34. Akasaki, Y. et al. Dysregulated FOXO transcription factors in articular cartilage in aging and osteoarthritis. Osteoarthritis Cartilage 22, 162-170 (2014).

Publisher's note Springer Nature remains neutral with regard to jurisdictional claims in published maps and institutional affiliations.

(c) The Author(s), under exclusive licence to Springer Nature Limited 2020 


\section{Methods}

No statistical methods were used to predetermine sample size. The experiments were not randomized, unless otherwise mentioned. The investigators were not blinded to allocation during experiments and outcome assessment.

\section{Mice}

C57BL/6J mice, 129/Sv mice (Janvier Labs), B6.Cg-Tg(CAG-eGFP) mice $^{35}$, B6.Cg-Tg(Colla1-cre/ERT2,-DsRed)1Smkm/J mice ${ }^{36}$, B6;129S4Sox $9 \mathrm{tm} 1.1 \mathrm{Tlu} / \mathrm{J}$ mice and B6.Cg-Tg(CAG-DsRed*MST)1Nagy/J mice (The Jackson Laboratory) were used in this study. Unless otherwise specified, both male and female mice were used for all experiments. All animal experiments were conducted according to the regulations and with approval of the Animal Ethics Committee of the KU Leuven.

\section{Mouse bone-healing models}

The femoral segmental bone-graft model was adapted from a previously described model ${ }^{8}$. Eight- to ten-week old male C57BL/6J mice were anaesthetized with a ketamine-xylazine mixture $(100 \mathrm{mg}$ per kg ketamine and $15 \mathrm{mg}$ per kg xylazine) and the right femur was exposed. A mid-diaphyseal 4-mm bone segment was excised with a $6.5-\mathrm{mm}$ diamond saw disk (Codema), briefly washed in saline to remove the bone marrow (periosteum not removed) and the segment was subsequently reimplanted in the defect (autograft). To investigate the contribution of donor cells, grafts were isolated from CAG-eGFP mice (periosteum not removed) and transplanted in wild-type littermates. To obtain devitalized allografts, 4 -mm bone segments were isolated from $129 / \mathrm{Sv}$ mice, washed in saline to remove the bone marrow, scraped to remove the periosteum, sterilized in $70 \%$ ethanol and frozen at $-80^{\circ} \mathrm{C}$ for at least 1 week. After graft implantation, the defect was stabilized with an intramedullary metal pin ( 22 gauge needle). To create a compromised host environment, a polycarbonate filter with a pore size of $30 \mu \mathrm{m}$ or $0.2 \mu \mathrm{m}$ (Sterlitech) was inserted between the muscle and the graft at the time of surgery.

The tibial fracture healing model was performed as previously described $^{5}$. For studies with the FFAR1/4 agonist GW9508 mice were treated daily by subcutaneously injecting $50 \mu$ of a $200 \mu \mathrm{M} \mathrm{GW} 9508$ (Cayman Chemical) solution or vehicle ( $0.2 \%$ DMSO in saline) at the fracture site. For fatty acid delivery, mice were treated daily by subcutaneously injecting $20 \mu \mathrm{l}$ corn oil (Sigma) or control solution (saline) at the fracture site. For studies with the FOXO inhibitor AS1842856, mice were treated daily by subcutaneously injecting $50 \mu \mathrm{l}$ of a $10 \mu \mathrm{M}$ AS1842856 (Calbiochem) solution or vehicle (0.1\% DMSO in saline) at the fracture site. For metabolite labelling experiments mice were injected intravenously with the fluorescent fatty acid analogue BODIPY $558 / 568$ C12 (Red-C12; Invitrogen) at $1 \mathrm{mg}$ per kg body weight and the fluorescent glucose analogue 2-( $N$-(7-nitrobenz-2-oxa-1,3-diazol-4-yl) amino)-2-deoxyglucose (2-NBDG; Invitrogen) at $12.5 \mathrm{mg}$ per $\mathrm{kg}$ body weight, 15 min before euthanasia. For skeletal stem cell transplantations, 100,000 cells (shCpt1a experiments) or 20,000 cells ( $\mathrm{shFoxo1}$ and shFoxo3a experiments) were resuspended in $5 \mu \mathrm{l}$ of a $5 \mathrm{mg} \mathrm{ml}^{-1}$ collagen gel (rat tail collagen type I, Corning) and transplanted at the fracture site at the time of surgery.

\section{Micro-computed tomography analysis}

Mice were euthanised at 2 or 4 weeks after surgery and grafted bones were isolated. For bone analysis, samples were scanned using the high resolution SkyScan 1172 micro-computed tomography (microCT) system (Bruker-microCT) at a pixel size of $10 \mu \mathrm{m}$ with $50 \mathrm{kV}$ tube voltage and $0.5 \mathrm{~mm}$ aluminium filter. To reduce the metal artefacts induced by the presence of the intramedullary pin, microCT projection data was reconstructed using an iterative reconstruction technique and projection completion ${ }^{37}$. Custom software was made in MeVisLab (MeVis Medical Solutions) to visualize and analyse the obtained microCT images.
The boundary between graft and callus was manually delineated and mineralized tissue was segmented using hysteresis thresholding. For visual representation grafts are represented in a different colour to callus and host bone. The coverage ratio was calculated as the percentage of the graft surface that is covered with callus by determining whether the normal line to the graft surface encounters mineralized callus, for each point of the graft surface.

For visualization and quantification of the vasculature, mice were anaesthetized with a ketamine-xylazine-heparin mixture $(100 \mathrm{mg}$ per $\mathrm{kg}$ ketamine, $15 \mathrm{mg}$ per kg xylazine and 1,000 U per kg heparin) and successively perfused with $10 \mathrm{ml}$ of heparinized saline $\left(100 \mathrm{U} \mathrm{ml}^{-1}\right), 10 \mathrm{ml}$ of a $10 \%$ neutral-buffered formalin solution, $10 \mathrm{ml}$ of saline and $5 \mathrm{ml}$ of a preheated $30 \%$ barium sulfate solution (Micropaque, Guerbet) containing $2 \%$ gelatine. After perfusion, animals were placed on ice for at least $1 \mathrm{~h}$ and subsequently kept at $4{ }^{\circ} \mathrm{C}$ overnight to allow the gelatine to solidify, before removing the grafted hindlimbs for dualenergy microCT analysis ${ }^{38,39}$. Two microCT scans of each sample were taken on the SkyScan 1172 microCT system with effective beam energy below ( $50 \mathrm{kV}$ tube voltage with $0.5 \mathrm{~mm}$ aluminium filter) and above (100 kV tube voltage with $0.5 \mathrm{~mm}$ aluminium and $0.038 \mathrm{~mm}$ copper filter) the K-edge energy of barium sulfate, both with an image pixel size of $5 \mu \mathrm{m}$. By combining the low and high energy acquisitions, an image of the (barium sulfate-perfused) vasculature only was reconstructed as described ${ }^{38,39}$ and a segmentation of the vasculature was obtained by thresholding this image. A segmentation of the bone was obtained by thresholding the bone and vasculature out of the low energy reconstruction and removing the calculated vasculature from it. After delineating a $250-\mu \mathrm{m}$-wide region of interest around the graft surface using a custom made MeVisLab software package, calculation of the number of blood vessels and the average vessel thickness was performed using the CTAn software (Bruker-microCT).

\section{Immunohistochemistry}

To isolate bones for histological analysis, mice were anaesthetized with ketamine-xylazine-heparin and perfused with $10 \mathrm{ml}$ of heparinized saline followed by $10 \mathrm{ml}$ of $2 \%$ paraformaldehyde in PBS. Isolated bones were further fixed in $2 \%$ paraformaldehyde overnight and decalcified in EDTA for 14 days at $4{ }^{\circ} \mathrm{C}$. Samples were either embedded in paraffin and sectioned at $4 \mu \mathrm{m}$, embedded in agarose for vibratome sections ( $100 \mu$ m thick) or embedded in NEG-50 frozen section medium (Richard-Allen Scientific) and sectioned at $7 \mu \mathrm{m}$ using the CryoJane Tape-Transfer System (Leica) for samples containing fluorescent protein-expressing cells. Staining with haematoxylin and eosin (H\&E) and safranin O, terminal deoxynucleotidyl transferase dUTP nick end labelling (TUNEL) staining and immunohistochemical staining for BrdU, CD31 and COL2 are routinely used in our laboratory and have all been described previously ${ }^{5,13,40-43}$. For SOX9, COL1, CPT1a, GLUT1 and FOXO3a immunohistochemical staining, sections were deparaffinised and blocked for $30 \mathrm{~min}$ in $0.1 \mathrm{M} \mathrm{Tris}-\mathrm{HCl}, 0.15 \mathrm{M}$ $\mathrm{NaCl}, \mathrm{pH} 7.6$ (TNT) with 0.5\% Blocking Reagent (NEN, PerkinElmer) and $20 \%$ normal goat serum (DAKO). Subsequently, sections were incubated overnight with a rabbit anti-SOX9 primary antibody (Novus Biologicals; NBP1-85551; 1:100), rabbit anti-COL1 primary antibody (Novus Biologicals; NB600-408; 1:100), rabbit anti-CPT1a primary antibody (Cell Signaling Technology; no. 12252; 1:50), rabbit antiGLUT1 primary antibody (Cell Signaling Technology; no. 12939; 1:100) or rabbit anti-FOXO3a primary antibody (Cell Signaling Technology, no. 2497, 1:100) diluted in TNT with $0.5 \%$ blocking reagent, followed by three washes with TNT containing 0.05\% Tween-20. Next, slides were incubated for $1 \mathrm{~h}$ with an Alexa Fluor 546- or Alexa Fluor 488-conjugated goat anti-rabbit secondary antibody (Invitrogen; A-11010 and A-11034) diluted 1:200 in TNT/0.5\% blocking reagent, washed and counterstained with Hoechst $33342\left(20 \mu \mathrm{g} \mathrm{ml}^{-1}\right.$ in PBS; Invitrogen). Stainings omitting the primary antibody were used as negative controls. 
Images were taken on a Zeiss Axioplan 2 light microscope, Zeiss LSM510-META NLO multi-photon confocal microscope or Zeiss LSM880 confocal laser scanning microscope. Histomorphometry was performed using the Zeiss AxioVision software, ImageJ software (National Institutes of Health) and CellProfiler software ${ }^{44}$. Quantification of blood vessels or proliferating cells was performed by respectively counting $\mathrm{CD} 1^{+}$vessels or $\mathrm{BrdU}^{+}$cells in a $250-\mu \mathrm{m}$-wide region of interest adjacent to the graft surface. Apoptotic or chondrogenic cells were quantified by respectively counting the number of TUNEL ${ }^{+}$ or SOX $9^{+}$cells and the total number of cells in a $0.015 \mathrm{~mm}^{2}$ region of interest near the graft surface at the centre of the graft. Quantification of cartilage was performed by outlining $\mathrm{COL}_{2}^{+}$or safranin $\mathrm{O}^{+}$areas within the total callus area (for fractures and grafts) or the central graft callus area (half of total graft length). Quantification of woven bone was performed by outlining areas of macroscopically-defined immature bone within the total callus area. Quantification of FOXO3a ${ }^{+}$nuclei was performed using the 'cell/particle counting and scoring' pipeline in CellProfiler, in a region of interest encompassing the total callus area (for fractures) or the central graft callus area (half of total graft length). For all quantifications, measurements were made on at least three different sections throughout the sample.

\section{Computational model of bone-graft healing}

We used a previously established multiscale computational framework of bone regeneration that quantitatively describes the interplay between cells, growth factors, nutrient levels and blood vessels ${ }^{10,11}$. In short, this multiscale model combines ten partial differential equations of the taxis-reaction-diffusion type at the tissue level with a discrete agent-based approach at the vascular level, including eight intracellular variables for the endothelial cells. At the tissue level, the model accounts for the various key processes of intramembranous and endochondral ossification that occur during the soft and hard callus phase of bone healing. The partial differential equations describe the evolution in time and space of the skeletal progenitor cell density, fibroblast density, chondrocyte density, osteoblast density, fibrous matrix density, cartilaginous matrix density, bone matrix density, osteochondrogenic growth factor concentration, vascular growth factor concentration and nutrient concentration. For simplification purposes, only one generic osteochondrogenic growth factor and one nutrient parameter is included in the computational model, which respectively represent the effects of multiple growth factors (for example, transforming growth factors or bone morphogenetic proteins) and nutrients (such as oxygen, glucose, amino acids or lipids) present during bone healing. The assumption is made that the net result of all growth factors present will be to promote chondrogenesis and osteogenesis, and thus if local levels of the osteochondrogenic growth factor reach a certain threshold (modelled using a sixth-order Hill function) it will induce differentiation of skeletal progenitor cells. The decision on whether the end result of this differentiation event is chondrogenic or osteogenic is made by the nutrient parameter. The influence of the generic osteochondrogenic growth factor on skeletal progenitor cell differentiation is promoting chondrogenic differentiation when local nutrient levels are low, and promoting osteogenic differentiation when local nutrient levels are high. Cell types that are considered at the tissue scale (skeletal progenitor cells, chondrocytes, osteoblasts, fibroblasts) can migrate (only skeletal progenitor cells and fibroblasts), proliferate, differentiate and produce growth factors (generic osteochondrogenic growth factor or angiogenic growth factor) and extracellular matrix (cartilage, bone or fibrous tissue). Blood vessels are modelled at both a cellular level (representing the developing vasculature with discrete endothelial cells) and an intracellular level (that defines the internal dynamics of every endothelial cell), and serve as the nutrient source. At the cellular level, the development of the discrete vascular tree (composed of endothelial cells) is determined by three different processes, that is, sprouting (the formation of a new branch, headed by a tip endothelial cell), vascular growth (the extension of the branch due to tip cell migration) and anastomosis (the fusion of two branches). An anastomosis between blood vessels allows for blood flow and the delivery of nutrients. The intracellular level considers a number of molecular players that govern endothelial cell movement (VEGFR2, DLL4, Notch and actin).

While the blood vessels are modelled discretely, continuous variables are used for nutrient density, bone density, cartilage density and fibrous tissue density (included in the model but not relevant for the current setup and therefore not shown). The colour scale for nutrients, bone and cartilage thus indicates a continuous gradient going from complete absence of a parameter (' 0 ' value; nutrients, bone or cartilage are not present at that location) to complete saturation of a parameter (' 1 ' value; a location is completely filled with nutrients, bone or cartilage). All values in between 0 and 1 represent partial filling of a location with a parameter. For the 'tissue' continuous variables (bone, cartilage, fibrous tissue), the sum of all tissues is 1 , meaning that if a location is completely filled with bone (value ' 1 '), no cartilage can exist at the same location (value ' 0 '). However, since the variables are continuous, a specific location can contain both a fraction of bone and a fraction of cartilage. Tissues, nutrients and blood vessels are modelled in separate spaces and can thus 'co-exist' in the same location. Since the nutrient parameter is also continuous, it has an independent scale going from no nutrients (value ' 0 ') to saturating levels of nutrients (value ' 1 ', which we define as the level of nutrients found inside a modelled blood vessel).

By adapting the geometry and boundary conditions to the bone-graft setup, the influence of a filter placed in between graft and muscle on the healing process can be predicted in silico. Detailed information on the equations, parameter values and implementation can be found in ref. ${ }^{11}$. Information on the boundary and initial conditions used in this study can be found in Extended Data Fig. 3.

\section{Isolation of primary cells}

Periosteal cells and trabecular osteoblasts were isolated from the long bones of 8-10-week-old mice as described ${ }^{13}$. For the isolation of periosteal cells, femurs and tibias were dissected free of muscle and connective tissue under sterile conditions. Subsequently, the epiphyses were protected from digestion by submerging them in 5\% low melting point agarose (SeaPlaque, Lonza) and periosteal cells were isolated by enzymatic digestion using $3 \mathrm{mg} \mathrm{ml}^{-1}$ collagenase II (Gibco) and $4 \mathrm{mg} \mathrm{ml}^{-1}$ dispase (Gibco) in $\alpha$-minimal essential medium ( $\alpha$-MEM; Gibco) supplemented with $1 \%$ penicillin/streptomycin ( 100 units $\mathrm{l}^{-1}$ and $100 \mu \mathrm{g} \mathrm{ml}^{-1}$ respectively; Gibco). Cells from the first digest $(10 \mathrm{~min})$ were discarded as they contain cells from remaining muscle and connective tissue, and periosteal cells were obtained by a subsequent $1 \mathrm{~h}$ digest. The cells were passed through a 70- $\mu$ m nylon mesh (BD Falcon), washed twice and cultured in $\alpha$-MEM with $1 \%$ penicillin/streptomycin and $10 \% \mathrm{FBS}$ (HyClone) in a humidified incubator at $37^{\circ} \mathrm{C}$ with $5 \% \mathrm{CO}_{2}$. For the isolation of trabecular osteoblasts, femurs and tibias were cleaned thoroughly to remove muscle, connective tissue and periosteum. Subsequently, bones were incubated in collagenase-dispase $\left(3 \mathrm{mg} \mathrm{ml}^{-1}\right.$ collagenase II and $4 \mathrm{mg} \mathrm{ml}^{-1}$ dispase in $\alpha$-MEM with $1 \%$ penicillin/streptomycin) for 20 min to remove remaining periosteal cells. Next, epiphyses were cut away, bone marrow was flushed out and the bone was cut into small pieces. Trabecular osteoblasts were isolated by incubating the bone fragments with collagenase-dispase for $30 \mathrm{~min}$. Cells were passed through a 70- $\mu \mathrm{m}$ nylon mesh, washed twice and cultured in $\alpha$-MEM supplemented with $1 \%$ penicillin/streptomycin and $10 \% \mathrm{FBS}$ at $37^{\circ} \mathrm{C}$ with $5 \% \mathrm{CO}_{2}$. Cells from passage $2-3$ were used for all experiments.

Growth plate-derived chondrocytes and calvarial osteoblasts were isolated from 3-5-day-old mice as described ${ }^{13,20}$. For murine growth plate-derived chondrocytes the resting zones of the growth plates from the distal femora and proximal tibiae were dissected free from surrounding tissue and pre-digested for $30 \mathrm{~min}$ with $1 \mathrm{mg} \mathrm{ml}^{-1} \mathrm{col}-$ lagenase II in $\alpha$-MEM with $1 \%$ penicillin/streptomycin on a shaker at 


\section{Article}

room temperature. Cartilage fragments were then washed twice and subsequently digested for $3 \mathrm{~h}$ in a $2 \mathrm{mg} \mathrm{ml}^{-1}$ collagenase II solution in $\alpha$-MEM with $1 \%$ penicillin/streptomycin on a shaker at $37^{\circ} \mathrm{C}$. The cell suspension was then filtered through a $40-\mu$ m nylon mesh, washed and cultured in $\alpha$-MEM supplemented with $1 \%$ penicillin/streptomycin and $10 \% \mathrm{FBS}$ at $37^{\circ} \mathrm{C}$ with $5 \% \mathrm{CO}_{2}$. Calvarial osteoblasts were prepared by 6 sequential 15-min digestions of calvaria from 3-5-day-old mice in PBS containing $1 \mathrm{mg} \mathrm{ml}^{-1}$ collagenase II and $2 \mathrm{mg} \mathrm{ml}^{-1}$ dispase. Cells isolated in fractions 2-6 were pooled and cultured in $\alpha$-MEM supplemented with $1 \%$ penicillin/streptomycin and $10 \% \mathrm{FBS}$ at $37^{\circ} \mathrm{C}$ with $5 \% \mathrm{CO}_{2}$. Cells from passage 2-3 were used for all experiments.

For isolation of rib chondrocytes, anterior rib cages were dissected from 5-day-old mice. Isolated rib cages were pre-digested on a shaker for $30 \mathrm{~min}$ at room temperature with $1 \mathrm{mg} \mathrm{ml}^{-1}$ collagenase II (Gibco) dissolved in $\alpha$-MEM supplemented with $1 \%$ penicillin/streptomycin. Rib fragments were subsequently digested for $3 \mathrm{~h}$ in a $2 \mathrm{mg} \mathrm{ml}^{-1}$ collagenase II solution in $\alpha$-MEM with $1 \%$ penicillin/streptomycin on a shaker at $37^{\circ} \mathrm{C}$. The obtained cell suspension of the second digest was filtered through a $40-\mu \mathrm{m}$ nylon mesh and single cells were recovered by centrifugation. Cells were cultured in a humidified incubator at $37^{\circ} \mathrm{C}$ with $5 \% \mathrm{CO}_{2}$ in $\alpha$-MEM supplemented with $1 \%$ penicillin/ streptomycin and $10 \%$ FBS. Cells from passage $2-3$ were used for all experiments.

Isolation of mouse skeletal stem cells was adapted from a previously described protocol ${ }^{16}$. Long bones of 3-5-day-old mice were dissected, muscle was cleared away carefully to preserve the periosteum and bones were minced using a scalpel. Bone fragments were then digested in $\alpha$-MEM supplemented with $3 \mathrm{mg} \mathrm{ml}^{-1}$ collagenase II, $4 \mathrm{mg} \mathrm{ml}^{-1}$ dispase (both from Gibco) and $100 \mathrm{U} \mathrm{ml}^{-1}$ DNase I (Sigma) at $37^{\circ} \mathrm{C}$ for 3 sequential 15 -min digests. Cell fractions were pooled and passed through a 70- $\mu \mathrm{m}$ nylon mesh, washed with PBS containing $2 \% \mathrm{FBS}$ and stained with antibodies against CD45, TER119, TIE2, CD105, CD90.2, CD249 (also known as 6C3) (BioLegend), CD51 (BD Pharmingen) and CD200 (eBioscience), and with the viability dye 7-aminoactinomycin D (7AAD; BD Pharmingen). Immunophenotyp-

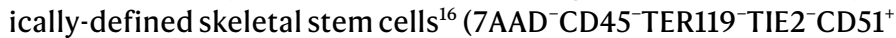

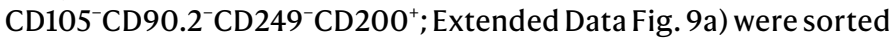
on a BD FACSAria II (BD Biosciences). Single colour controls were used to set compensations and fluorescence minus one controls were used to set gates. Sorted cells were cultured in a humidified incubator at $37^{\circ} \mathrm{C}$ with $2 \% \mathrm{O}_{2}$ and $7.5 \% \mathrm{CO}_{2}$ in $\alpha$-MEM supplemented with $1 \%$ penicillin/streptomycin and $10 \%$ FBS. For metabolic analyses, skeletal stem cells were grown in atmospheric $\mathrm{O}_{2}$ levels with $5 \% \mathrm{CO}_{2}$ to enable direct comparison with other cell types. Cells from passage 2-3 were used for all experiments. For flow cytometric analysis of culture-expanded skeletal stem cells, cells were gated again for the CD $51^{+} \mathrm{CD} 105^{-} \mathrm{CD} 90.2^{-} \mathrm{CD} 249^{-} \mathrm{CD} 200^{+}$population to limit analysis to the stem cell fraction.

For the isolation of skeletal muscle-derived cell populations, hindlimb skeletal muscles, including quadriceps, soleus, gastrocnemius and tibialis anterior, were dissected from 8-week-old CAG-DsRed mice, minced using a scalpel and digested in $\alpha$-MEM medium supplemented with $3 \mathrm{mg} \mathrm{ml}^{-1}$ collagenase II, $4 \mathrm{mg} \mathrm{ml}^{-1}$ dispase and $100 \mathrm{U} \mathrm{ml}^{-1}$ DNase I at $37^{\circ} \mathrm{C}$ for $60 \mathrm{~min}$. Every $15 \mathrm{~min}$, samples were pipetted up and down vigorously using a $10-\mathrm{ml}$ serological pipette to break up tissue fragments. Cell suspensions were passed through a $70-\mu$ m nylon mesh, washed with PBS containing 2\% FBS and stained with antibodies against CD45, TER119, CD31, F4/80 and CD146 (BioLegend), and with 7AAD (BDPharmingen). Immunophenotypically-defined macrophages $\left(7 \mathrm{AAD}^{-} \mathrm{CD}^{-} 5^{+} \mathrm{F} 4 / 80^{+}\right.$), endothelial cells $\left(7 \mathrm{AAD}^{-} \mathrm{CD}^{-} 5^{-} \mathrm{TER}^{-} 119^{-} \mathrm{F} 4 / 80^{-} \mathrm{C}\right.$

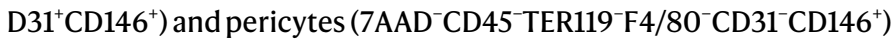
(Extended Data Fig. 9b) were sorted on a BD FACSAria II. Single colour controls were used to set compensations and fluorescence minus one controls were used to set gates. Sorted cells were used for co-cultures with periosteal cells in micromasses.

\section{Cell lines}

The $\mathrm{C} 3 \mathrm{H} 10 \mathrm{~T} 1 / 2$ cell line, used as a skeletal progenitor cell model ${ }^{12}$, was obtained from the RIKEN Cell Bank and cultured in a humidified incubator at $37^{\circ} \mathrm{C}$ with $5 \% \mathrm{CO}_{2}$ in $\alpha$-MEM with $1 \%$ penicillin/streptomycin and $10 \%$ FBS. Cells were routinely tested and found negative for mycoplasma contamination.

\section{Nutrient-deprivation assays}

Cells were seeded at 3,000 cells per $\mathrm{cm}^{2}$ in basal DMEM (glucose- and glutamine-free; Gibco) supplemented with $1 \%$ penicillin/streptomycin, $5 \mathrm{mM} \mathrm{D}$-(+)-glucose (Sigma-Aldrich), $2 \mathrm{mM} \mathrm{L-glutamine} \mathrm{(Gibco),} 1 \mathrm{mM}$ sodium pyruvate (Gibco) and $10 \%$ dialysed FBS (HyClone). After $24 \mathrm{~h}$, cells were washed with PBS and switched to control medium (basal DMEM with $1 \%$ penicillin/streptomycin, $5 \mathrm{mM}$ glucose, $2 \mathrm{mM} \mathrm{L-glu-}$ tamine, $1 \mathrm{mM}$ sodium pyruvate and $10 \%$ dialysed FBS), SD medium (basal DMEM with $1 \%$ penicillin/streptomycin, $5 \mathrm{mM}$ glucose, $2 \mathrm{mM}$ L-glutamine, $1 \mathrm{mM}$ sodium pyruvate and $1 \%$ dialysed FBS), glucosedeprivation medium (basal DMEM with $1 \%$ penicillin/streptomycin, $0.5 \mathrm{mM}$ glucose, $2 \mathrm{mM}$ L-glutamine, $1 \mathrm{mM}$ sodium pyruvate and $10 \%$ dialysed FBS), glutamine-deprivation medium (basal DMEM with $1 \%$ penicillin/streptomycin, $5 \mathrm{mM}$ glucose, $0.2 \mathrm{mM}$ L-glutamine, $1 \mathrm{mM}$ sodium pyruvate and $10 \%$ dialysed FBS), CND medium (basal DMEM with $1 \%$ penicillin/streptomycin, $0.5 \mathrm{mM}$ glucose, $0.2 \mathrm{mML}$-glutamine, $1 \mathrm{mM}$ sodium pyruvate and $1 \%$ dialysed FBS) or LRS medium (basal DMEM with $1 \%$ penicillin/streptomycin, $5 \mathrm{mM}$ glucose, $2 \mathrm{mM} \mathrm{L-glu-}$ tamine, $1 \mathrm{mM}$ sodium pyruvate and $10 \%$ lipid-reduced FBS). LRS was made by mixing FBS with fumed silica (Sigma) at $20 \mathrm{mg} \mathrm{ml}^{-1}$ for $3 \mathrm{~h}$ at room temperature, followed by centrifugation at $2,000 \mathrm{~g}$ for $15 \mathrm{~min}$ and filtration of the supernatant through a $0.45-\mu \mathrm{m}$-pore-size filter.

In certain experiments cultures were supplied with actinomycin D (transcription inhibitor; Sigma-Aldrich), cycloheximide (translation inhibitor; Sigma-Aldrich), chloroquine (lysosomal inhibitor; Sigma-Aldrich) or etomoxir (CPT1 inhibitor; Merck-Millipore) at the concentrations indicated in the text. For lipid rescue experiments, SD medium was supplemented with very low density lipoproteins (VLDL; Calbiochem) at a concentration of $607 \mu \mathrm{g}$ triglycerides per ml FBS, palmitic or oleic acid (Sigma-Aldrich) at the indicated concentrations or a mixture of PUFAs $(10 \mu \mathrm{M}$ linoleic acid, $15 \mu \mathrm{M} \alpha$-linolenic acid, $10 \mu \mathrm{M}$ arachidonic acid and $15 \mu \mathrm{M}$ docosahexaenoic acid; all from SigmaAldrich). Triglycerides were incubated in FBS for 30 min at $37^{\circ} \mathrm{C}$ and fatty acids (dissolved in ethanol) were complexed to fatty acid-free bovine serum albumin (BSA) (Sigma-Aldrich) for $1 \mathrm{~h}$ at $37^{\circ} \mathrm{C}$ before adding to the culture medium, as described previously ${ }^{45}$. All supplements were added at the start of the experiment and were present for the entire duration of the cultures.

\section{Differentiation assays}

To assess chondrogenic differentiation, 150,000 periosteal cells were resuspended in $10 \mu \mathrm{l}$ of control medium and seeded as micromasses in the middle of a 24-well plate. Cells were allowed to attach for $1 \mathrm{~h}$ at $37^{\circ} \mathrm{C}$, after which $0.5 \mathrm{ml}$ of control, SD or LRS medium containing $10 \mathrm{ng} \mathrm{ml}^{-1}$ recombinant human TGF $\beta 1$ (Peprotech), $50 \mu \mathrm{M} \mathrm{L}$-ascorbic acid 2-sulfate (Sigma-Aldrich) and $20 \mu \mathrm{M}$ Y-27632 (Rho kinase inhibitor; Axon Medchem $)^{46}$ was added to the wells. Medium was refreshed every other day and after 9 days micromasses were either stained with alcian blue or used for RNA isolation. For chondrogenic differentiation in the presence of muscle-derived cells micromasses were made using 100,000 periosteal cells derived from Sox9-GFP mice and 50,000 skeletal muscle-derived macrophages, endothelial cells, pericytes or unsorted cells obtained from CAG-DsRed mice.

For chondrogenic differentiation in pellets 200,000 periosteal cells were placed in a $5-\mathrm{ml}$ polystyrene tube in $1 \mathrm{ml}$ of control, SD or LRS medium containing $10 \mathrm{ng} \mathrm{ml}^{-1}$ recombinant human TGF $\beta 1$ (Peprotech) and $50 \mu \mathrm{ML}$-ascorbic acid 2-sulfate (Sigma-Aldrich), supplemented with 
vehicle ( $1 \%$ ethanol in $4 \%$ fatty acid-free BSA in saline), $60 \mu \mathrm{M}$ oleate or a mixture of PUFA (10 $\mu \mathrm{M}$ linoleic acid, $15 \mu \mathrm{M} \alpha$-linolenic acid, $10 \mu \mathrm{M}$ arachidonic acid and $15 \mu \mathrm{M}$ docosahexaenoic acid) complexed to fatty acid-free BSA. Tubes were centrifuged for $5 \mathrm{~min}$ at $500 \mathrm{~g}$ and placed in a humidified incubator at $37^{\circ} \mathrm{C}$. Medium was changed every 3 days and after 21 days pellets were fixed in $4 \%$ paraformaldehyde for 10 min and processed for paraffin histological sectioning.

For osteogenic differentiation, periosteal cells were seeded cells at 30,000 cells per $\mathrm{cm}^{2}$ in control medium and cultured for 3 days in order to reach full confluence. Cells were then switched to control, SD or LRS medium containing $50 \mu \mathrm{ML}$-ascorbic acid 2-sulfate and $10 \mathrm{mM}$ $\beta$-glycerophosphate(Sigma-Aldrich).After 21 days, cellswere either stained with alizarin red $S$ to detect mineralization or used for RNA isolation.

\section{Metatarsal cultures}

Metatarsal rudiments were dissected from E16.5 Col1a1-cre/ERT2, -DsRed embryos and stripped of skin. The middle three metatarsals were kept together as triads and cultured for 7 days on a Falcon insert membrane (pore size $0.4 \mu \mathrm{m}$ ) in 12-well plates in $1 \mathrm{ml}$ of BGJb culture medium (Gibco) supplemented with $25 \mathrm{\mu g} \mathrm{ml}^{-1} \mathrm{~L}$-ascorbic acid 2-sulfate, $10 \mathrm{mM} \beta$-glycerophosphate, and FBS $(10 \% \text { or } 1 \%)^{40}$. When indicated a mixture of PUFAs $(10 \mu \mathrm{M}$ linoleic acid, $15 \mu \mathrm{M} \alpha$-linolenic acid, $10 \mu \mathrm{M}$ arachidonic acid and $15 \mu \mathrm{M}$ docosahexaenoic acid) complexed to fatty acid-free BSA or vehicle ( $1 \%$ ethanol in $4 \%$ fatty acid-free BSA in saline) was added to the culture medium. At the end of the cultures the metatarsals were fixed overnight in $2 \%$ paraformaldehyde in PBS and processed for histochemistry or immunohistochemistry.

\section{Flow cytometry}

Cell death was detected using annexin V-FITC and propidium iodide (Dead Cell Apoptosis Kit; Invitrogen), or using active caspase 3-FITC (FITC Active Caspase-3 Apoptosis Kit; BD Pharmingen). Proliferation was assessed by staining with a PE-conjugated mouse anti-Ki-67 antibody (BD Pharmingen; \#556027; 1:10) and Hoechst $33342\left(40 \mu \mathrm{g} \mathrm{ml}^{-1}\right.$; Invitrogen) after fixation and permeabilization of the cells (BD Cytofix/ Cytoperm Kit, BD Biosciences). Intracellular SOX9 levels were quantified by staining with an Alexa Fluor 647-conjugated rabbit anti-SOX9 antibody (Cell Signaling Technology; no. 71273;1:100) after fixation and permeabilization of the cells. Gating for SOX $9^{\text {high }}$ cells was set to have approximately $10 \%$ SOX $9^{\text {high }}$ cells in control conditions. Single colour controls were used to set compensations and fluorescence minus one controls were used to set gates.

\section{Immunocytochemistry}

For immunofluorescence microscopy, cells grown on coverslips were fixed with $4 \%$ paraformaldehyde, permeabilized with $0.5 \%$ Triton-X100 in PBS and blocked with PBS containing 5\% BSA, 5\% normal goat serum and $0.5 \%$ Tween-20. Next, cells were incubated overnight at $4{ }^{\circ} \mathrm{C}$ with primary antibodies (rabbit anti-FOXO1, Cell Signaling Technology, no. 2880, 1:100; rabbit anti-FOXO3a, Cell Signaling Technology, no. $2497,1: 100)$ in blocking buffer, followed by three washes with PBS/ Tween-20. Slides were subsequently incubated for $2 \mathrm{~h}$ with secondary antibodies (Alexa Fluor 488-conjugated goat anti-rabbit; 1:500) in PBS containing 5\% BSA and $0.5 \%$ Tween-20, washed and counterstained with Hoechst 33342 . Stainings omitting the primary antibody were used as negative controls.

For staining of lipid droplets with 1,6-diphenyl-1,3,5-hexatriene (DPH), cells grown on coverslips were washed with PBS and fixed with 3.7\% formaldehyde in PBS. DPH staining solution was prepared by diluting a $2 \mathrm{mM}$ DPH (Sigma-Aldrich) stock (in DMSO) in PBS to a final concentration of $4 \mu \mathrm{M}$ as previously described ${ }^{47}$. Cells were stained with DPH for $30 \mathrm{~min}$, washed and nuclei were counterstained using TO-PRO-3 (Molecular Probes).

For tracking lipid movement between lipid droplets and mitochondria, cells were incubated with the fluorescent fatty acid analogue
BODIPY 558/568 C12 (Red-C12; Invitrogen) at $1 \mu \mathrm{M}$ in culture medium for $16 \mathrm{~h}\left(\right.$ ref. $\left.^{24}\right)$. Cells were then washed three times with culture medium, incubated for $1 \mathrm{~h}$ in culture medium to allow the fluorescent lipids to incorporate into lipid droplets, and then chased for the time indicated in control or SD medium. Mitochondria were labelled with $100 \mathrm{nM}$ MitoTracker Deep Red FM (Invitrogen) for 30 min before the end of the experiment. Cells were fixed and lipid droplets were stained with DPH as described above.

For measurement of autophagic flux, cells grown on coverslips were transfected with $1 \mu \mathrm{g}$ of an RFP-GFP-LC 3 tandem construct ${ }^{48}$ using the $X$-tremeGENE HP transfection reagent (Roche) according to the manufacturer's instructions. After $24 \mathrm{~h}$, cells were washed with PBS and used for subsequent experiments. Since the GFP-LC3 loses fluorescence owing to lysosomal acidic and degradative conditions but the RFP-LC3 does not, autophagosomes in the cell are seen as green-yellow puncta, whereas autophagolysosomes are red.

Images were taken on a Zeiss LSM510-META NLO multi-photon confocal microscope or Zeiss LSM880 confocal laser scanning microscope, and prepared using Adobe Photoshop CS5 (Adobe) and ImageJ. LC3 puncta and $\mathrm{DPH}^{+}$lipid droplets per cell were counted manually in ImageJ, while overlap between MitoTracker and Red-C12 in manually delineated cells was performed using the 'co-localization' plugin for ImageJ after thresholding of individual frames.

\section{Western blot analysis}

Total cell lysates were obtained by lysing cells in $25 \mathrm{mM}$ Tris-HCl buffer ( $\mathrm{pH} 7.6$ ) containing $150 \mathrm{mM} \mathrm{NaCl}, 1 \% \mathrm{NP}-40,1 \%$ sodium deoxycholate, $0.1 \%$ SDS, $1 \times$ cOmplete protease inhibitor cocktail (Roche) and $1 \times$ PhosSTOP phosphatase inhibitor cocktail (Roche). For cytoplasmic and nuclear extracts, cells were first lysed in $20 \mathrm{mM}$ Hepes (pH 7.9) containing $10 \mathrm{mM} \mathrm{KCl}, 1.5 \mathrm{mM} \mathrm{MgCl}_{2}, 1 \mathrm{mM}$ EDTA, 0.5\% NP40, $1 \mathrm{mM}$ DTT, $1 \mathrm{mM} \mathrm{Na}_{3} \mathrm{VO}_{4}, 20 \mathrm{mM} \mathrm{NaF} 1 \mathrm{mMPMSF}, 5 \mu \mathrm{g} \mathrm{ml}^{-1}$ aprotinin, $5 \mu \mathrm{g} \mathrm{ml}^{-1}$ leupeptin and $0.33 \mathrm{\mu g} \mathrm{ml}^{-1}$ antipain. Following $15 \mathrm{~min}$ incubation at $4{ }^{\circ} \mathrm{C}$, the cell lysates were passed 10 times through a 26 gauge needle. After centrifugation for 1 min at $18,000 g$, the supernatant (cytoplasmic proteins) was removed and the pellet containing the nuclear protein fraction was resuspended in $50 \mathrm{mM}$ Hepes ( $\mathrm{pH} 7.9)$ containing $500 \mathrm{mM}$ $\mathrm{NaCl}, 1 \% \mathrm{NP} 40,5 \mu \mathrm{g} \mathrm{ml}^{-1}$ aprotinin, $5 \mu \mathrm{g} \mathrm{ml}^{-1}$ leupeptin and $0.33 \mu \mathrm{g} \mathrm{ml}^{-1}$ antipain, and sonicated. Proteins $(10 \mu \mathrm{g}$, except for detection of LC3 for which $20 \mu$ g was used) were separated by SDS-PAGE and transferred to a nitrocellulose membrane (GE Healthcare). Membranes were blocked with $5 \%$ dry milk in Tris-buffered saline with $0.1 \%$ Tween-20 for $30 \mathrm{~min}$ at room temperature and incubated overnight at $4{ }^{\circ} \mathrm{C}$ with primary antibodies (rabbit anti-SOX9, Novus Biologicals, NBP1-85551,1:2,000; rabbit anti-FOXO1, Cell Signaling Technology, no. 2880, 1:1,000; rabbit anti-FOXO3a, Cell Signaling Technology, no. 2497, 1:1,000; rabbit anti-LC3B, Cell Signaling Technology, no. 3868, 1:500; mouse anti$\beta$-actin, Sigma, A5441, 1:10,000; mouse anti-lamin A/C, Santa Cruz Biotechnology, sc-376248, 1:5,000) diluted in blocking buffer. Signals were detected by enhanced chemiluminescence (Perkin Elmer) after incubation with HRP-conjugated secondary antibodies (DAKO). For gel source data, see Supplementary Fig. 1.

\section{Metabolic assays}

Glucose and lactate levels in culture medium were measured on a AU640 Chemistry Analyzer (Beckman Coulter). Glucose consumption was calculated by subtracting the remaining amount of glucose in the culture medium after $24 \mathrm{~h}$ of incubation with cells from the amount of glucose in unspent medium, and normalized for time and for cell number via DNA quantification. In a similar way, lactate secretion was calculated by subtracting lactate levels in unspent medium from the levels in medium incubated for $24 \mathrm{~h}$ with cells. Oxygen consumption was determined on a Seahorse XF24 Analyzer (Seahorse Bioscience) using 50,000 cells per well. The assay medium was unbuffered DMEM (Sigma) supplemented with $5 \mathrm{mM}$ D-glucose and $2 \mathrm{mM} \mathrm{L-glutamine,}$ 
$\mathrm{pH}$ 7.4. For quantification of FAO-linked oxygen consumption the difference in OCR before and after injection of etomoxir $(100 \mu \mathrm{M}$ final concentration) was calculated ${ }^{22}$.

For measurement of glycolysis, cells were incubated for $6 \mathrm{~h}$ in growth medium containing $0.3 \mu \mathrm{Ci} \mathrm{ml}^{-1}\left[5-{ }^{3} \mathrm{H}\right] \mathrm{D}$-glucose (PerkinElmer). The culture medium was then transferred into glass vials sealed with rubber caps. ${ }^{3} \mathrm{H}_{2} \mathrm{O}$ was captured in hanging wells containing a Whatman paper soaked with $\mathrm{H}_{2} \mathrm{O}$ over a period of $48 \mathrm{~h}$ at $37^{\circ} \mathrm{C}$ to reach saturation $^{49}$. Radioactivity was determined in the paper by liquid scintillation counting and values were normalized to DNA content.

For glucose oxidation, cells were incubated for $6 \mathrm{~h}$ in growth medium containing $0.6 \mu \mathrm{Ci} \mathrm{ml}^{-1}\left[6^{-14} \mathrm{C}\right] \mathrm{D}$-glucose (PerkinElmer). To stop cellular metabolism, $250 \mu$ l of a $2 \mathrm{M}$ perchloric acid solution was added and wells were covered with a Whatman paper soaked with $1 x$ hyamine hydroxide. ${ }^{14} \mathrm{CO}_{2}$ released during the oxidation of glucose was absorbed into the paper overnight at room temperature. Radioactivity in the paper was determined by liquid scintillation counting, and values were normalized to DNA content ${ }^{49}$.

FAO was measured after incubation of the cells with $3 \mu \mathrm{Ci} \mathrm{ml}{ }^{-1}$ $\left[9,10-{ }^{3} \mathrm{H}\right]$ palmitate (PerkinElmer), complexed to BSA, for $2 \mathrm{~h}$. Then, the culture medium was transferred into glass vials sealed with rubber caps. ${ }^{3} \mathrm{H}_{2} \mathrm{O}$ was captured in hanging wells containing a Whatman paper soaked with $\mathrm{H}_{2} \mathrm{O}$ over a period of $48 \mathrm{~h}$ at $37^{\circ} \mathrm{C}$. Radioactivity in the paper was determined by liquid scintillation counting, and values were normalized to DNA content ${ }^{49}$.

\section{Metabolite diffusion assay}

Diffusion rates were measured in custom-designed diffusion chambers according to a previously established protocol ${ }^{50}$. Chambers were fabricated in a polydimethylsiloxane (PDMS) device on a glass substrate with medium reservoirs that contained fluorescent tracer molecules. 2-NBDG (342 Da) and BODIPY FL C16 (FL-C16; Invitrogen) complexed to fatty acid-free BSA $(66.5 \mathrm{kDa})$ were used as fluorescent analogues to evaluate the diffusion rates of glucose and fatty acids, respectively, in separate runs. Tracer movement was assessed in square borosilicate glass capillaries with an inner width of $0.8 \mathrm{~mm}$ and wall thickness of $0.16 \mathrm{~mm}$ (VitroCom). Collagen type I gels $\left(5 \mathrm{mg} \mathrm{ml}^{-1}\right)$ containing periosteal cells ( 5 million per $\mathrm{ml}$ ) were polymerized within the capillaries, after which the capillaries were connected to the PDMS reservoirs which initiated the diffusion process resulting from a concentration gradient between the tracer saturated medium reservoir $(250 \mu \mathrm{M} 2-\mathrm{NBDG}$ or $25 \mu \mathrm{M}$ FL-C16 complexed to $25 \mu \mathrm{M}$ BSA) and the tracer-free capillary. Tracer gradients within the capillaries were imaged on a confocal fluorescence laser scanning microscope (FV1000, Olympus) equipped with a UPLSAPO $10 \times$ air objective (NA 0.40 ) focused on the middle plane of the collagen gel. Focus drift was compensated using an IX81-ZDC module that focuses a 785-nm laser on the glass capillary surface to stably reproduce the focus position for each capillary position and for every acquisition time point. Images were acquired as a time series with 10-min intervals over a total period of $5 \mathrm{~h}$, at $37^{\circ} \mathrm{C}$. Tracer-free collagen gels were visualized to correct the image sequences for any background fluorescence intensity. A tracer saturated collagen gel was visualized during each diffusion experiment to compensate for potential photobleaching of tracer and to normalize the gradient profiles for further processing. Image sequences were processed in ImageJ. Diffusion rates were obtained by least squares fitting an analytical solution of Fick's second diffusion law to the resulting averaged axial intensity profiles in MATLAB (MathWorks) ${ }^{50}$.

\section{Gene targeting}

To silence Sox9, Cpt1a, Atg5, Foxo1 or Foxo3a, we transduced cells in the presence of $8 \mu \mathrm{g} \mathrm{ml}^{-1}$ polybrene (Sigma-Aldrich), with a lentivirus carrying a shRNA against SOX9[51] (Addgene plasmid repository no. 40645; multiplicity of infection (MOI) 50), CPT1a (MISSION, Sigma-Aldrich; MOI25) or ATG5 (MISSION, Sigma-Aldrich; MOI25), or concomitantly with shRNAs against Foxo1 and Foxo3a (MISSION, Sigma-Aldrich; each at MOI 25). To overexpress SOX9 we transduced cells, in the presence of $8 \mu \mathrm{g} \mathrm{ml}^{-1}$ polybrene, with a lentivirus carrying a SOX9-overexpression plasmid[51] (Addgene plasmid repository no.36979; MOI150). A nonsense scrambled (Scr) shRNA sequence or empty vector was used as a negative control. After $24 \mathrm{~h}$, virus-containing medium was changed to normal culture medium and $48 \mathrm{~h}$ later, cells were used for further experiments. Target knockdown was confirmed by western blot.

To silence expression of Foxo genes using CRISPR-Cas9, we transduced Cas9-expressing C3H10T1/2 cells (Cas9: Addgene plasmid repository no. 48139$)^{52}$, with a lentivirus carrying doxycycline-inducible sgRNAs against Foxo1 (GenBank accession number NM_019739) (5'-TTGTAAAGGTGTCTTCACGGGGG-3') and Foxo3a (GenBank accession number NM_019740) (5'-CATTCTGAACGCGCATGAAGCGG-3') (doxycycline-inducible plasmid: Addgene plasmid repository no. $70183)^{53}$. Cells were cultured in the presence of doxycycline $\left(250 \mathrm{ng} \mathrm{ml}^{-1}\right)$ for $72 \mathrm{~h}$ before experiments.

\section{Quantification of active FOXO levels}

Levels of active FOXO were measured using the TransAM FKHR (FOXO1) DNA-binding ELISA (Active Motif) on nuclear protein extracts, and normalized to total nuclear protein input as measured by bicinchoninic acid assay (Pierce BCA Protein Assay Kit; Thermo Scientific).

\section{Total RNA extraction and RT-qPCR analysis}

Total RNA from cultured cells was extracted using the RNeasy Mini Kit (Qiagen). Total RNA from cortical bone (femurs of eight-weekold mice, cleaned and flushed to remove bone marrow) and cartilage (growth plates dissected from the distal femur and proximal tibia of three-day-old pups) was extracted using TRIzol (Invitrogen) followed by RNA clean-up using the RNeasy Mini Kit. mRNA was reverse transcribed using Superscript II Reverse Transcriptase (Invitrogen). Reverse transcription with quantitative PCR (RT-qPCR) was performed on the 7500 Fast Real-Time PCR System (Applied Biosystems). Specific forward and reverse oligonucleotide primers were used either in conjunction with SYBR Green dye (Cpt1a, Acadm, Acadl and Myod (also known as Myod1)) or with FAM-TAMRA conjugated probes (all others). The following primers and probes were used: Sox 9 (GenBank accession number NM_011448): 5'-TCTGGAGGCTGCTGAACGA-3' (forward), 5'-TCCGTTCTTCACCGACTTCCT-3' (reverse), 5'-FA M-CAGCACAAGAAAGACCACCC-TAMRA-3' (probe); Col2a1 (GenBank accession number NM_031163): 5'-AGAACATCACCTACCACTGTA AGAACA-3' (forward), 5'-TGACGGTCTTGCCCCACTT-3' (reverse), 5'-FAM-CCTTGCTCATCCAGGGCTCCAATG-TAMRA-3' (probe); Acan (GenBank accession number NM_001361500): 5'-GCATGAGAGA GGCGAATGGA-3' (forward), 5'-CTGATCTCGTAGCGATCTTTCTTCT-3' (reverse), 5'-FAM-CTGCAATTACCAGCTGCCCTTCACGT-TAMRA-3' (probe); Runx2 (GenBank accession number NM_001146038): 5'-TACCAGCCACCGAGACCAA-3' (forward), 5'-AGAGGCTGTT TGACGCCATAG-3' (reverse), 5'-FAM-CTTGTGCCCTCTGTT GTAAATACTGCTTGCA-TAMRA-3' (probe); Ocn (GenBank accession number NM_007541): 5'-GGCCCTGAGTCTGACAAAGC-3' (forward), 5'-GCTCGTCACAAGCAGGGTTAA-3' (reverse), 5'-FAM-ACAGACTCC GGCGCTACCTTGGAGC-TAMRA-3' (probe); Pparg (GenBank accession number NM_001127330): 5' - CCCAATGGTTGCTGATTACAAA-3' (forward), 5'-AATAATAAGGTGGAGATGCAGGTTCT-3' (reverse), 5'-FA M- CTGAAGCTCCAAGAATACCAAAGTGCGATC-TAMRA-3' (probe); Myod (GenBank accession number NM_010866): 5'-GCGCGAGTCCA GGCCAGG-3' (forward), 5'-CGACTCTGGTGGTGCATCTGC-3' (reverse); Slc2a1 (GenBank accession number NM_011400): 5'-GGGCATGTGCTT CCAGTATGT-3' (forward), 5'-ACGAGGAGCACCGTGAAGAT-3' (reverse), 5'-FAM-CAACTGTGCGGCCCCTACGTCTTC-TAMRA-3' (probe); Pfkfb3 (GenBank accession number NM_001177757): Mm.PT.51.16600796 (Integrated DNA Technologies); Ldha (GenBank accession number NM_010699): 5'-TTCATCATTCCCAACATTGTCAA-3' (forward), 
5'-CACTGATTTTCCAAGCCACGTA-3' (reverse), 5'-FAM-AGTCCACAC TGCAAGCTGCTGATCGTC-TAMRA-3' (probe); Cpt1a (GenBank accession number NM_013495): 5'-GCCCATGTTGTACAGCTTCC-3' (forward), 5'-TTGGAAGTCTCCCTCCTTCA-3' (reverse); Acadm (GenBank accession number NM_007382): 5'-TTTCGAAGACGTCAGAGTGC-3' (forward), 5'-TGCGACTGTAGGTCTGGTTC-3' (reverse); Acadl (GenBank accession number NM_007381): 5'-TCTTTTCCTCGGAGCATGACA-3' (forward), 5'-GACCTCTCTACTCACTTCTCCAG-3' (reverse). Expression levels were analysed using the $2^{-\Delta \Delta C t}$ method and were normalized for the expression of the housekeeping gene $A c t b$.

\section{RNA-seq, gene expression quantification and enrichment analysis of transcription binding motifs}

In brief, total RNA was extracted from C 3 H10T1/2 cells seeded in sixwell plates using TRIzol. Polyadenylated RNA enrichment, reverse transcription and stranded library preparation were done using the KAPA stranded mRNA-seq kit (Roche). The first 50 bases of these libraries were sequenced on a HiSeq4000 (Illumina) and mapped to the murine genome (build mm10) using TopHat v.2.1.1. ${ }^{54}$. Read counts were processed using EdgeR v.3.20.955 to identify genes differentially expressed between cells that were serum-starved (1\% FBS) and cells that were control-treated (10\% FBS). The top-100 most-significantly upregulated genes upon serum starvation (at a 1\% false discovery rate, differential expression in EdgeR is assessed for each gene using an exact test analogous to Fisher's exact test, but adapted for overdispersed data $^{55}$ ) were analysed for motif enrichment using $\mathrm{i}$-cisTarge $\mathrm{t}^{26}$.

\section{Single-cell RNA-seq- of mouse long bone}

The single-cell RNA-seq dataset of the mouse long bone and bone marrow stroma was generated previously and detailed information on cell isolation, cell sorting, library preparation, RNA-seq and data processing is provided in the original manuscript ${ }^{21}$. A set of 40 genes involved in $\mathrm{FAO}$ and 34 genes involved in glycolysis was curated from the Gene Ontology database (http://software.broadinstitute.org/gsea/msigdb) and the Kyoto Encyclopedia of Genes and Genomes (KEGG) database (http://www.genome.jp/kegg). Gene expression was calculated as the fraction of its unique molecular identifier (UMI; random barcode) count with respect to total UMI in the cell and then multiplied by 10,000 . We denoted it as transcripts per 10,000 transcripts (TP10K).

\section{ChIP-qPCR}

ChIP-qPCR was performed as described ${ }^{56}$. In brief, $3 \mathrm{~h}$ after serum deprivation, $\mathrm{C} 3 \mathrm{H} 10 \mathrm{~T} 1 / 2$ cells were fixed using $1 \%$ formaldehyde, washed and collected by centrifugation $\left(1,000 \mathrm{~g}\right.$ for $5 \mathrm{~min}$ at $\left.4{ }^{\circ} \mathrm{C}\right)$. The pellet was resuspended in RIPA buffer $(50 \mathrm{mM}$ Tris- $\mathrm{HCl} \mathrm{pH} 8,150 \mathrm{mM} \mathrm{NaCl}$, 2 mMEDTA, $1 \%$ Triton-X100, 0.5\% sodium deoxycholate, $1 \%$ SDS and $1 \%$ protease inhibitors), homogenized, incubated on ice for $10 \mathrm{~min}$ and sonicated. The samples were centrifuged $\left(16,000 \mathrm{~g}\right.$ for $10 \mathrm{~min}$ at $\left.4{ }^{\circ} \mathrm{C}\right)$ and from the supernatant sheared chromatin was used as input (1/30), and on the remainder of the chromatin immunoprecipitation was performed with an anti-FOXO1 antibody (rabbit anti-FOXO1, Abcam, ab39670) or an anti-FOXO3a antibody (rabbit anti-FOXO3a, Abcam, ab12162). After precipitation using Pierce Protein A/G Magnetic Beads (Thermo Fisher Scientific), followed by RNA and protein digestion, DNA was purified using Agencourt AMPure XP (Beckman Coulter) according to the manufacturer's instructions. RT-qPCR was performed using SYBR GreenER qPCR SuperMix Universal (Thermo Fisher Scientific) and specific primers for the Sox 9 promoter region (5'-TGTGGGCATATTGGCTTCT-3' (forward), 5'-GGTTAAACTGGGAAGACTCATGG-3' (reverse)).

\section{Statistical analysis}

All numerical results are reported as mean \pm s.e.m. Statistical significance of the difference between experimental groups was analysed by two-tailed Student's $t$-test, one-way, two-way or three-way ANOVA with Bonferroni post hoc test (as indicated in the figure legends and source data files) using the GraphPad Prism software. Differences were considered statistically significant for $P<0.05$. In the studies performed in cell lines in culture, all experiments were independently repeated at least three times. Experiments using primary cells were performed with at least three biological replicates. Western blots were independently repeated at least twice. Mice for experiments were randomly allocated to groups. All numerical values used for graphs and detailed statistical analysis can be found in the source data files.

\section{Reporting summary}

Further information on research design is available in the Nature Research Reporting Summary linked to this paper.

\section{Data availability}

The bulk RNA-seq data that support the findings of this study have been deposited in ArrayExpress with the accession number E-MTAB-7564. The single-cell RNA-seq data were generated previously ${ }^{21}$ and are deposited in the Gene Expression Omnibus with accession number GSE128423. A portal for exploring the entire atlas is available at https:// portals.broadinstitute.org/single_cell/study/mouse-bone-marrowstroma-in-homeostasis. Source Data for Figs. 1-4 and Extended Data Figs. 1-8 are provided with the paper. All other data supporting the findings of this study are available within the paper.

\section{Code availability}

The full code used for the computational model of bone-graft healing is available from the authors upon request. More background information on the development of the model can be found in our previous publications $^{10,11}$.

35. Hadjantonakis, A. K., Gertsenstein, M., Ikawa, M., Okabe, M. \& Nagy, A. Generating green fluorescent mice by germline transmission of green fluorescent ES cells. Mech. Dev. 76, 79-90 (1998).

36. Ouyang, Z. et al. Prx1 and 3.2kb Col1a1 promoters target distinct bone cell populations in transgenic mice. Bone 58, 136-145 (2014)

37. Nuyts, J. et al. Iterative reconstruction for helical CT: a simulation study. Phys. Med. Biol. 43, 729-737 (1998)

38. Depypere, M. et al. An iterative dual energy CT reconstruction method for a K-edge contrast material. Proc. SPIE 7961, 79610M (2011).

39. Vandersmissen, I. et al. Endothelial Msx1 transduces hemodynamic changes into an arteriogenic remodeling response. J. Cell Biol. 210, 1239-1256 (2015).

40. Maes, C. et al. Soluble VEGF isoforms are essential for establishing epiphyseal vascularization and regulating chondrocyte development and survival. J. Clin. Invest. 113, 188-199 (2004).

41. Stiers, P. J., van Gastel, N., Moermans, K., Stockmans, I. \& Carmeliet, G. An ectopic imaging window for intravital imaging of engineered bone tissue. JBMR Plus 2, 92-102 (2018).

42. Stegen, S. et al. Osteocytic oxygen sensing controls bone mass through epigenetic regulation of sclerostin. Nat. Commun. 9, 2557 (2018).

43. Stiers, P. J. et al. Inhibition of the oxygen sensor PHD2 enhances tissue-engineered endochondral bone formation. J. Bone Miner. Res. 34, 333-348 (2019).

44. McQuin, C. et al. CellProfiler 3.0: Next-generation image processing for biology. PLoS Biol. 16, e2005970 (2018).

45. Daniëls, V. W. et al. Cancer cells differentially activate and thrive on de novo lipid synthesis pathways in a low-lipid environment. PLoS One 9, e106913 (2014).

46. Eyckmans, J., Lin, G. L. \& Chen, C. S. Adhesive and mechanical regulation of mesenchymal stem cell differentiation in human bone marrow and periosteum-derived progenitor cells. Biol. Open 1, 1058-1068 (2012).

47. Ranall, M. V., Gabrielli, B. G. \& Gonda, T. J. High-content imaging of neutral lipid droplets with 1,6-diphenylhexatriene. Biotechniques 51, 35-42 (2011).

48. Kimura, S., Noda, T. \& Yoshimori, T. Dissection of the autophagosome maturation process by a novel reporter protein, tandem fluorescent-tagged LC3. Autophagy 3, 452-460 (2007).

49. Schoors, S. et al. Fatty acid carbon is essential for dNTP synthesis in endothelial cells. Nature 520, 192-197 (2015).

50. Lambrechts, D. et al. A causal relation between bioluminescence and oxygen to quantify the cell niche. PLoS ONE 9, e97572 (2014).

51. Guo, W. et al. Slug and Sox 9 cooperatively determine the mammary stem cell state. Cell 148, 1015-1028 (2012).

52. Ran, F. A. et al. Genome engineering using the CRISPR-Cas9 system. Nat. Protoc. 8, 22812308 (2013).

53. Aubrey, B. J. et al. An inducible lentiviral guide RNA platform enables the identification of tumor-essential genes and tumor-promoting mutations in vivo. Cell Rep. 10, 1422-1432 (2015).

54. Trapnell, C., Pachter, L. \& Salzberg, S. L. TopHat: discovering splice junctions with RNAseq. Bioinformatics 25, 1105-1111 (2009). 


\section{Article}

55. Robinson, M. D., McCarthy, D. J. \& Smyth, G. K. edgeR: a Bioconductor package for differential expression analysis of digital gene expression data. Bioinformatics $\mathbf{2 6}$ 139-140 (2010).

56. Stegen, S. et al. HIF-1a promotes glutamine-mediated redox homeostasis and glycogendependent bioenergetics to support postimplantation bone cell survival. Cell Metab. 23, 265-279 (2016).

Acknowledgements We thank K. Moermans, I. Stockmans, C. MacGillivray and H. Soled for technical assistance, A. Nagy for the CAG-eGFP mice, S. Murakami for the Col1a1-cre/ERT2 and DsRed mice, T. Yoshimori for the RFP-GFP-LC3 plasmid, R. A. Weinberg for the pLKO.1-shmSOX9-5 lentiviral plasmid, M. Mazzone for hypoxic glove box use, the histology core of the Harvard Department of Stem Cell and Regenerative Biology for histology services, the FACS cores of the KU Leuven and the Harvard Department of Stem Cell and Regenerative Biology for access to the flow cytometers, and the Cell Imaging Core and the Molecular Imaging and Photonics division of the KU Leuven and the Harvard Center for Biological Imaging for access to the confocal microscopes. This work was supported by grants from the Research Fund Flanders (FWO; G096414, GOA4216N and GOB3418N to G.C.), KUL grant C24/17/07 (G.C.) grants from the European Research Council (ERC 308223 to H.V.O., ERC 279100 to L.G. and ERC 269073 to P.C.), and long-term structural Methusalem funding by the Flemish Government (P.C.). N.v.G. is funded by BOF-KU Leuven GOA project 3M120209. P.J.S. is a fellow from the Agency for Innovation by Science and Technology in Flanders (IWT). S. Stegen, A.C. and Dennis L. are postdoctoral fellows of the FWO. V.W.D. is a fellow of the FWO and the Flemish League against Cancer (VLK). This work is part of Prometheus, the KU Leuven R\&D Division of Skeletal Tissue Engineering.
Author contributions N.v.G. and G.C. conceived the study. N.v.G., S. Stegen, G.E., S. Schoors, P.-J.S., Dennis L., S.T. and A.S. performed the in vitro experiments. N.v.G. performed the in vivo experiments. A.C. performed the in silico experiments. V.W.D. and J.V.S. contributed to the design and execution (V.W.D.) of lipid-rescue experiments. N.B. and D.P. performed and analysed the single-cell RNA-seq experiment. M.D. and F.M. contributed to the design and execution (M.D.) of microCT analyses. R.V.L. and A.S. performed histology. P.A. contributed to the design and interpretation of autophagy experiments. N.v.G., A.C., L.G. and H.V.O. contributed to the design and interpretation of in silico experiments. Diether L. and B.T. contributed to the design, execution and interpretation of RNA-seq experiments. P.C. contributed to the design and interpretation of metabolic analyses. D.T.S. contributed to the design and interpretation of in vivo experiments. P.A., J.V.S., P.C. and D.T.S. provided reagents N.v.G., S. Stegen and G.C. designed the experiments and interpreted data. N.v.G. and G.C. wrote the manuscript. All authors agreed on the final version of the manuscript.

Competing interests The authors declare no competing interests.

Additional information

Supplementary information is available for this paper at https://doi.org/10.1038/s41586-020 2050-1.

Correspondence and requests for materials should be addressed to G.C.

Peer review information Nature thanks Thomas Clemens, Michael T. Longaker and the other, anonymous, reviewer(s) for their contribution to the peer review of this work.

Reprints and permissions information is available at http://www.nature.com/reprints. 
a

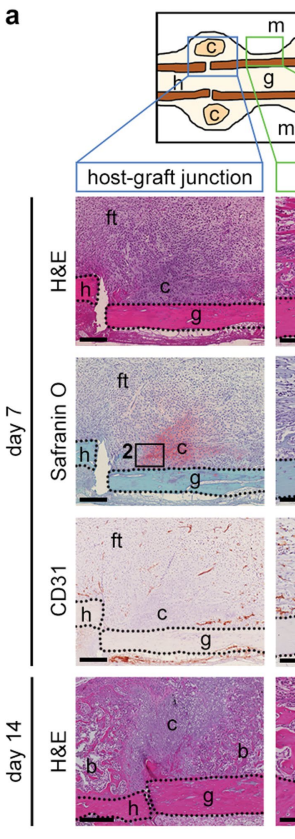

C

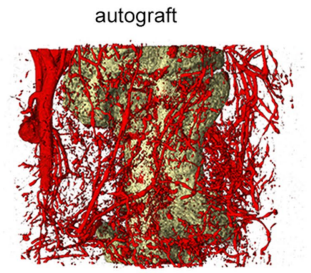

Extended Data Fig. 1 Removal of periosteum reduces bone formation and callus vascularization. a, Histological characterization of the mouse boneautograft healing model. At the host-graft junction cartilage (safranin $\mathrm{O}^{+}$) is formed at PFD7. Note absence of CD31 ${ }^{+}$blood vessels in these regions. Near the graft centre new woven bone (bright pink on H\&E staining) is deposited, cartilage is absent and blood vessels are abundant. By PFD14, the cartilage at the host-graft junction is gradually being replaced by bone, while the woven bone near the graft centre appears mature (representative images of four mice). Scale bars, $200 \mu \mathrm{m}$ in host-graft junction images, $100 \mu \mathrm{m}$ in graft-centre images, $50 \mu \mathrm{m}$ in magnifications. b, MicroCT-based visualization and quantification of newly formed bone around control autografts, autografts b
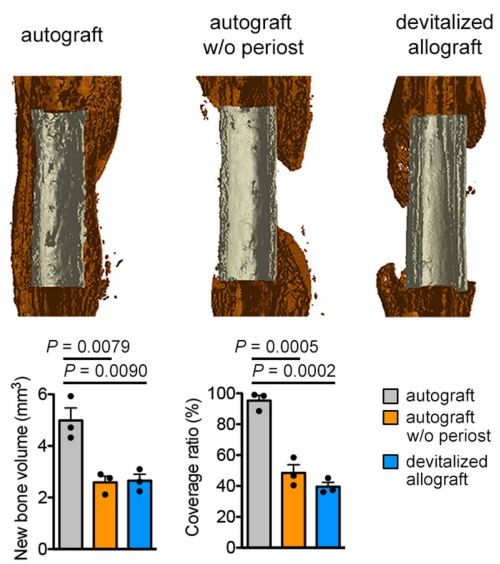

$P=0.0005$

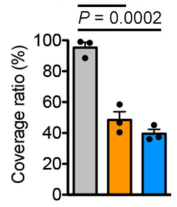

$\square$ autograft

w/o periost

$\square$ devitalized
allograft

ऐo
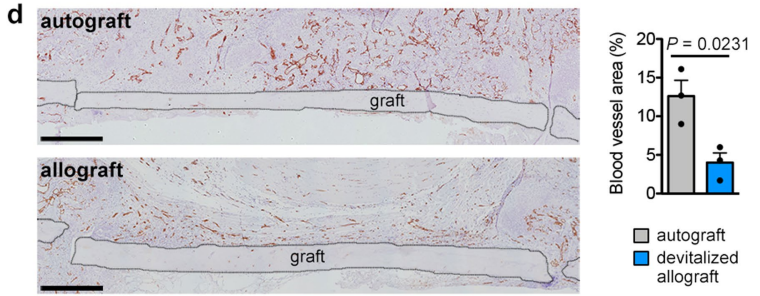

devitalized allograft
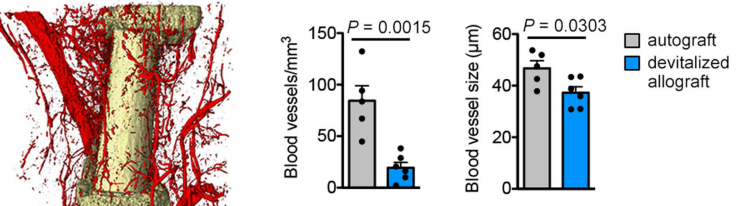

from which the periosteum was removed or devitalized allografts (no living cells) at PFD28 $(n=3$ mice). Coverage ratio represents percentage of graft surface covered by new bone. c, Dual-energy microCT-based visualization and quantification of vascularization in a $250-\mu \mathrm{m}$-wide region around autografts and allografts at PFD14 ( $n=5$ mice for autograft, $n=6$ mice for devitalized allograft).d, CD31 immunohistochemical visualization and quantification of vascularization in a $250 \mu \mathrm{m}$-wide region around autografts and allografts at PFD14 ( $n=3$ mice). Scale bars, $500 \mu \mathrm{m}$. b, bone; c, cartilage; $\mathrm{ft}$, fibrous tissue; g, graft; $h$, host; $m$, muscle; $p$, periosteum. Data are mean \pm s.e.m.; one-way ANOVA with Bonferroni post hoc test (b), two-tailed Student's $t$-test (c, d). 


\section{Article}

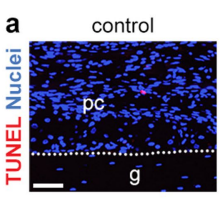

filter 0.2

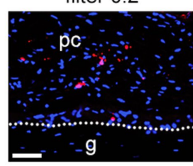

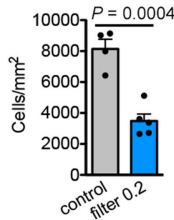

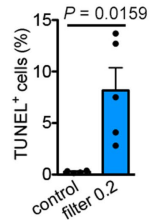

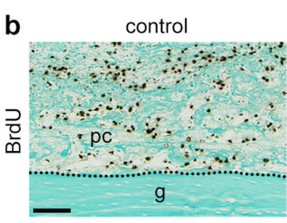
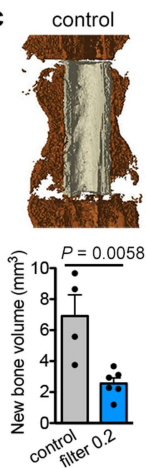

control
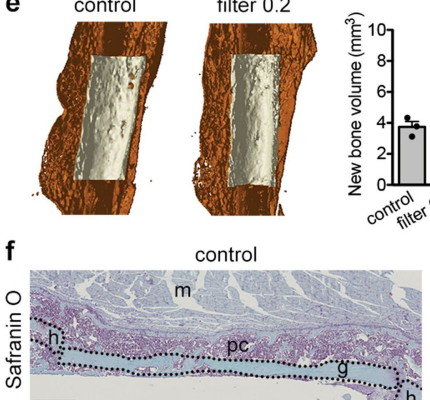

filter 0.2
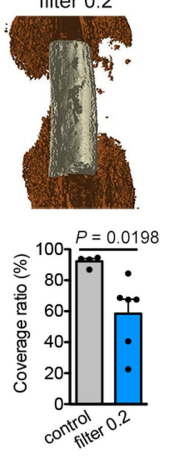

filter 0.2 filter 0.2
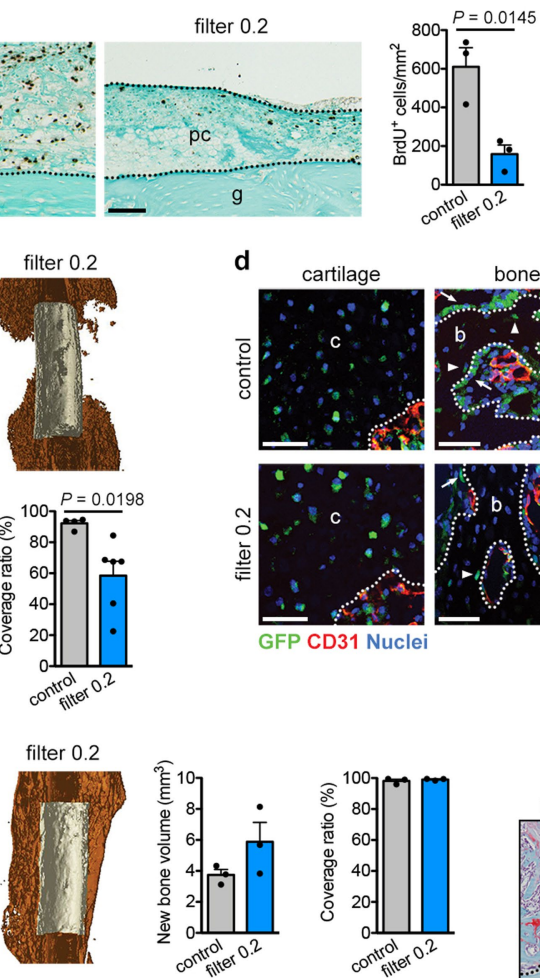

GFP CD31 Nucle
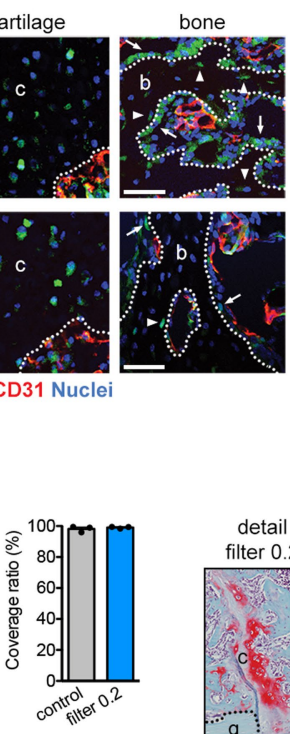

filter 0.2

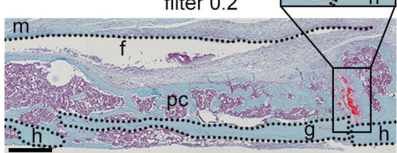

Extended Data Fig. 2 | Reducing vascularization alters but does not prevent bone healing. a, Histological visualization and quantification of apoptotic cells ( $\mathrm{TUNEL}^{+} ; n=4$ mice for control, $n=5$ mice for filter 0.2 ) in the callus of grafts with or without a filter $(0.2 \mu \mathrm{m}$ pore size $)$ at PFD7. Scale bars, $50 \mu \mathrm{m}$. b, Histological visualization and quantification of proliferating $\left(\mathrm{BrdU}^{+} ; n=3\right.$ mice) cells in the callus of grafts with or without a filter $(0.2 \mu \mathrm{m}$ pore size $)$ at PFD7. Scale bars, $100 \mu \mathrm{m}$.c, MicroCT-based visualization and quantification of newly formed bone around control grafts or grafts surrounded by a filter $(0.2 \mu \mathrm{m}$ pore size) at PFD14 ( $n=4$ mice for control, $n=6$ mice for filter 0.2$)$. Coverage ratio represents percentage of graft surface covered by new bone. d, Cell tracing of donor periosteal cells during healing of bone grafts, derived from CAG-eGFP mice, with or without filter $(0.2 \mu \mathrm{m}$ pore size $)$ at PFD14 showing equal contribution of donor cells to cartilage in both conditions, but reduced contribution of donor cells to bone near the graft ends. Arrows, $\mathrm{GFP}^{+}$ osteoblasts; arrowheads, $\mathrm{GFP}^{+}$osteocytes; representative images of three mice. Scale bars, $50 \mu \mathrm{m}$. e, MicroCT-based visualization and quantification of newly formed bone around control grafts or grafts surrounded by a filter $(0.2 \mu \mathrm{m}$ pore size $)$ at PFD28 ( $n=3$ mice).f, Histological analysis of autografts with or without a filter $(0.2 \mu \mathrm{m}$ pore size $)$ at PFD28 showing comparable callus morphology and composition, although remaining cartilage islands (detail image) were seen when a filter was present but not in the callus of control grafts (representative images of three mice). Scale bars, $500 \mu \mathrm{m}$. Data are mean \pm s.e.m.; two-tailed Student's $t$-test. 

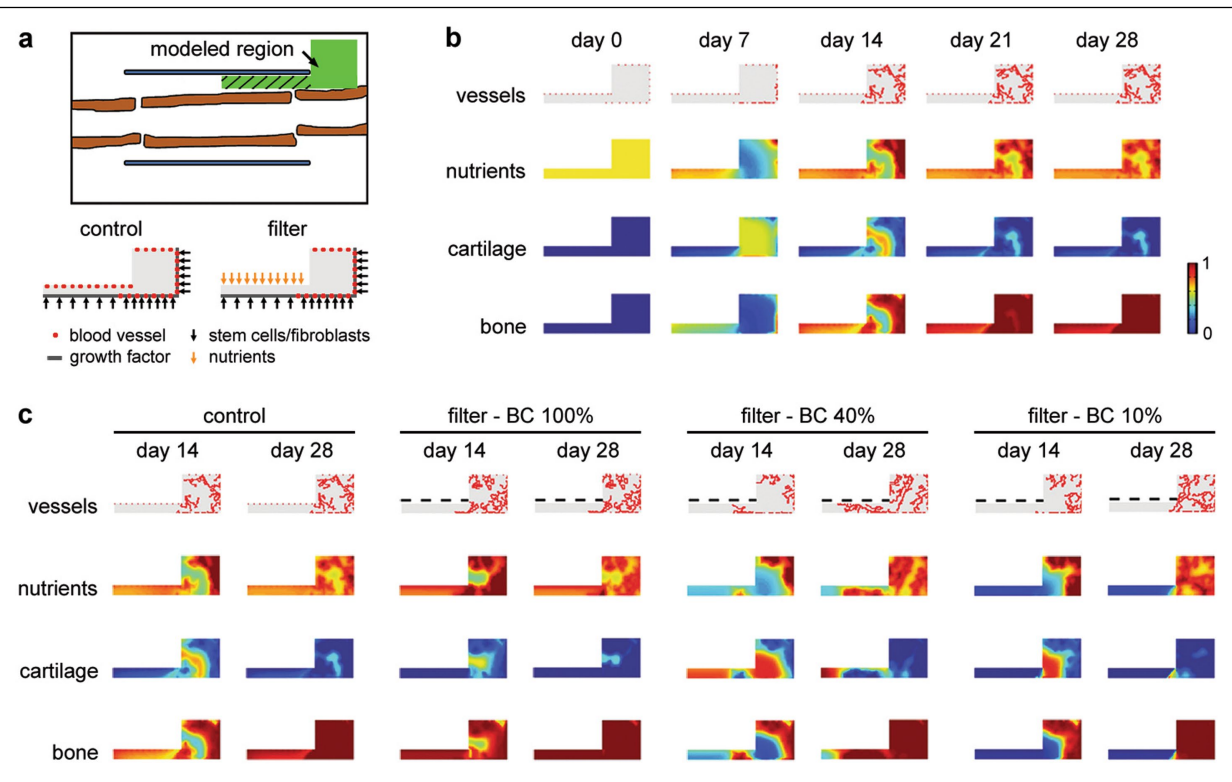

d

e
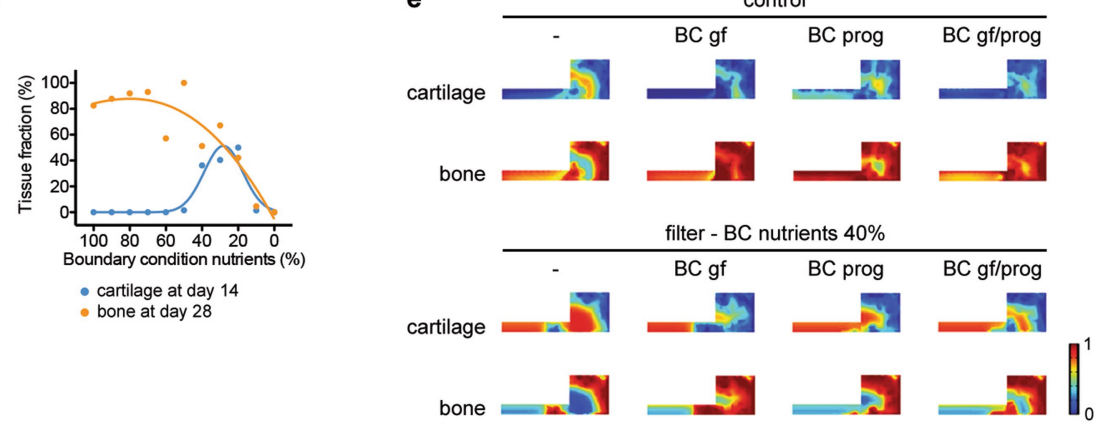

Extended Data Fig. 3 | In silico modelling supports a role for nutritional stress in chondrogenic commitment. Application of a previously described computational model of bone repair ${ }^{10,11}$ to the bone-graft healing setup. In this model, the behaviour (survival, proliferation, differentiation and tissue formation) of skeletal progenitor cells, chondrocytes, osteoblasts and fibroblasts is dependent on the local supply of nutrients by blood vessels, in addition to the presence of growth factors, extracellular matrix and the cell density. a, Schematic overview (top) of the modelled region shown in green. The hatched area represents the graft callus. At the start of the simulation the modelled region was filled with loose fibrous tissue matrix, growth factors, stem cells, osteoblasts, fibroblasts and nutrients, representing the fracture haematoma. Overview of the Dirichlet boundary conditions (bottom) showing the starting points of blood vessels and the sites of release of cells and growth factors (and nutrients for the condition with filter) during the healing process. b, Application of the model to the normal bone graft (that is, blood vessels can come from the muscle side). Heat map-based visualization of blood vessel, nutrient, cartilage and bone distribution in the modelled region at different time points shows that the model correctly predicts the spatiotemporal progression of the bone-healing process. Nutrients and tissue fractions are expressed on a non-dimensional scale ranging from 0 (absence) to 1 (saturation).c, d, Application of the model to bone-graft healing in the presence of a filter placed in between graft and muscle (that is, blood vessels cannot come from the muscle side) with visual representation (c) and quantification (d) of the different tissue fractions in the modelled region. Quantification was performed only in the left rectangle of the modelled region, as indicated by the hatched area in a, representing the graft callus. The amount of nutrients that can pass through the filter (the boundary condition (BC)) was varied between $100 \%$ (the maximum amount that can be supplied by the vasculature, applied to the whole filter length, resulting in similar nutrient distributions as in the control) and $0 \%$. When nutrient supply through the filter is set at $20-40 \%$, the model correctly recapitulates the chondrogenic switch in the central region of the graft as observed in vivo. When nutrient supply through the filter was $>40 \%$, the cells in the central graft region differentiated directly into osteoblasts, and a supply of nutrients $<20 \%$ induced massive cell death and completely prevented tissue formation and graft healing. e, Visual representation of the effect of additional growth factor (gf) diffusion and/or progenitor cell (prog) migration from the filter side on cartilage and bone fractions at day 14. The control situation (no filter) is shown on the top and the filter situation with a boundary condition for nutrients of $40 \%$ is shown on the bottom. No large effect of these additional boundary conditions on the healing response was observed. 

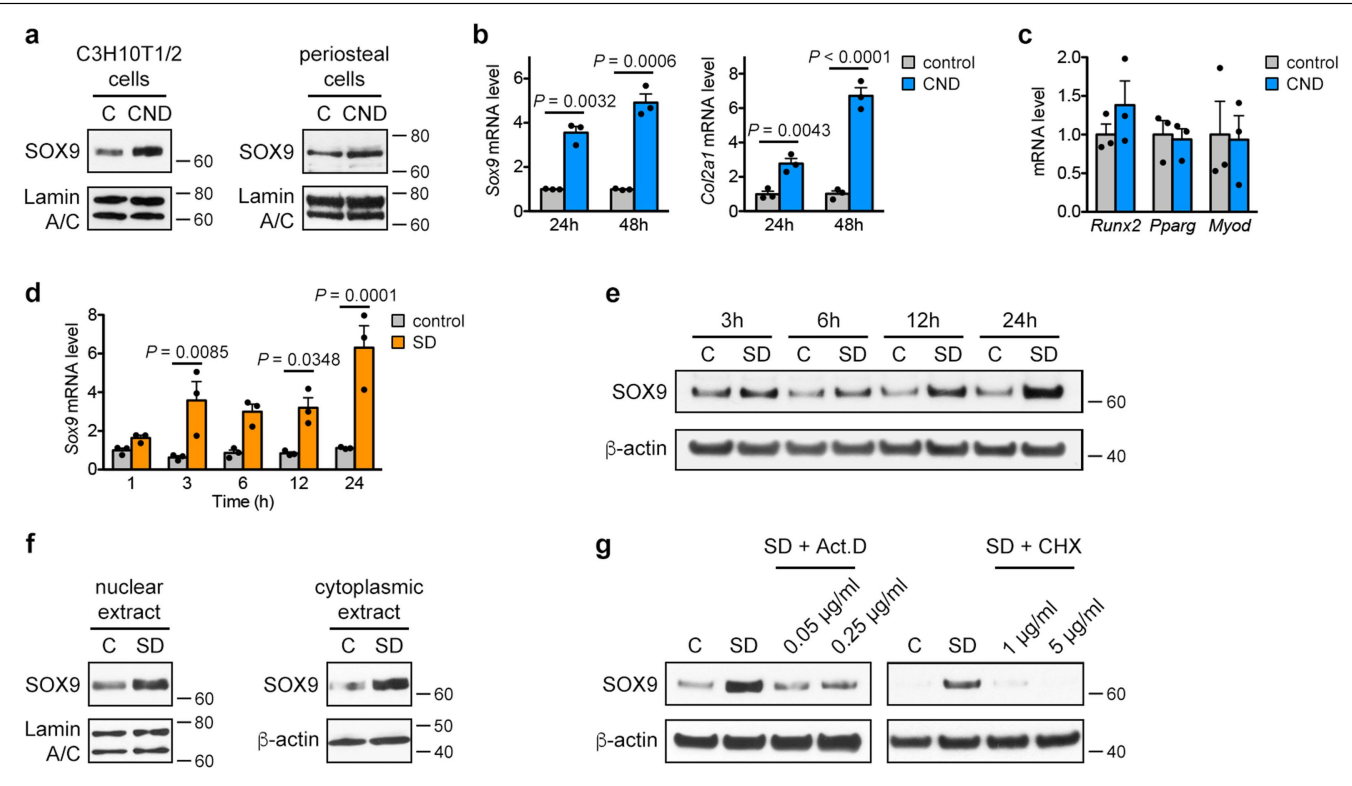

f

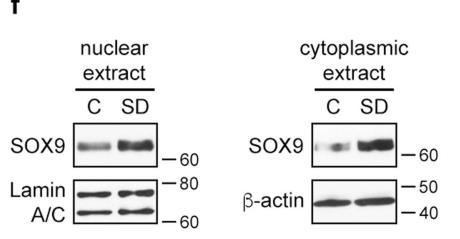

h

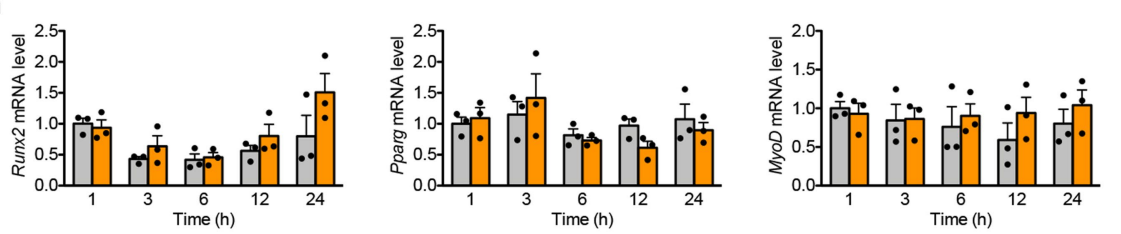

i

$$
\begin{gathered}
\text { C SD } \\
\text { SOx9 }=-60 \\
\text { Lamin }=-80 \\
\text { A/C }-60
\end{gathered}
$$

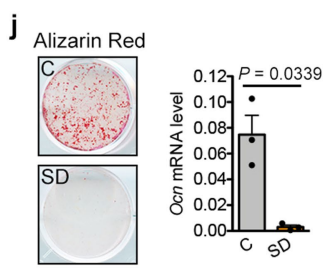

k
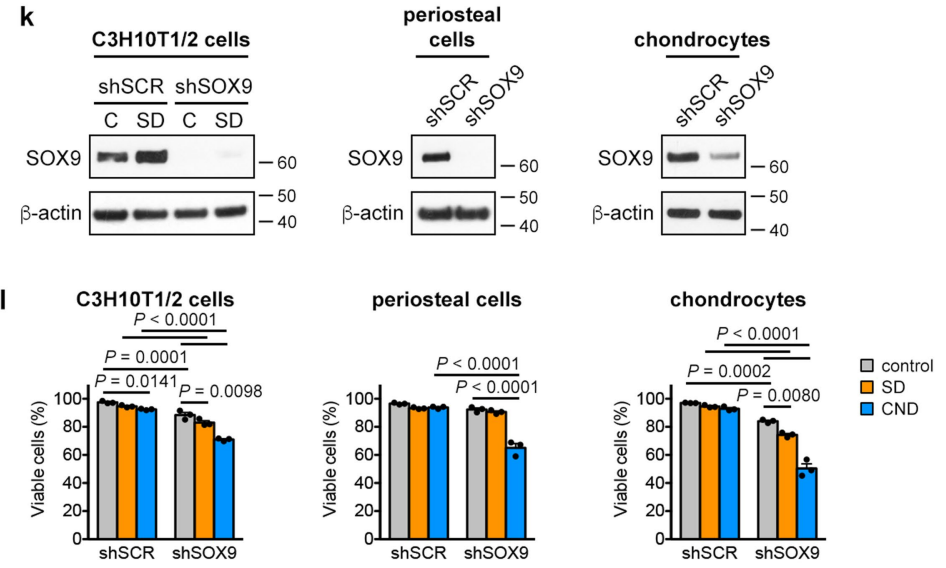

Extended Data Fig. 4 | See next page for caption. 
Extended Data Fig. 4 | Skeletal progenitors resist nutritional stress via induction of SOX9. a, Immunoblot detection of nuclear SOX9 in C3H10T1/2 cells and periosteal cells exposed for $24 \mathrm{~h}$ to control or CND medium, with lamin $\mathrm{A} / \mathrm{C}$ as loading control ( $n=2$ independent experiments). b, mRNA levels of Sox 9 and Col2a 1 in periosteal cells exposed for the indicated times to control or CND medium (relative to control; $n=3$ biologically independent samples). c, mRNA levels of runt-related transcription factor 2 (Runx2; osteogenic lineage), peroxisome proliferator-activated receptor $\gamma$ (Pparg; adipogenic lineage) and $M y o d$ (myogenic lineage) in periosteal cells exposed for $48 \mathrm{~h}$ to control or CND medium (relative to control; $n=3$ biologically independent samples). d, mRNA levels of Sox 9 in C3H10T1/2 cells exposed for the indicated times to control or SD medium (relative to control, $n=3$ independent experiments).e, Immunoblot detection of total SOX9 in C3H10T1/2 cells exposed for different durations to control or SD medium, with $\beta$-actin as loading control ( $n=2$ independent experiments). f, Immunoblot detection of nuclear and cytoplasmic SOX9 in C3H10T1/2 cells exposed for $6 \mathrm{~h}$ to control or SD medium, with lamin $\mathrm{A} / \mathrm{C}$ or $\beta$-actin as loading control $(n=2$ independent experiments). g, Immunoblot detection of SOX9 in total cell protein extracts of C3H10T1/2 cells exposed for $6 \mathrm{~h}$ to control medium, SD medium or SD medium supplemented with different concentrations of the transcription inhibitor actinomycin D (Act. D) or the translation inhibitor cycloheximide (CHX).

Detection of $\beta$-actin was used as loading control $(n=2$ independent experiments).h, mRNA levels of Runx2, Pparg and Myod in C3H10T1/2 cells exposed for the indicated times to control or SD medium (relative to control, $n=3$ independent experiments). $\mathbf{i}$, Immunoblot detection of nuclear SOX9 in periosteal cells exposed for $24 \mathrm{~h}$ to control or SD medium with lamin $\mathrm{A} / \mathrm{C}$ as loading control ( $n=3$ biologically independent samples). $\mathbf{j}$, Osteogenic differentiation of periosteal cells in control or SD medium, assessed by visualization of mineral deposits (alizarin red staining) and quantification of $O c n$ mRNA levels (relative to $A c t b, n=3$ biologically independent samples). k, Immunoblot detection of SOX9 in total cell protein extracts of C3H10T1/2 cells (in control or SD medium), periosteal cells and growth plate-derived chondrocytes transduced with shSox 9 or shScr, with $\beta$-actin as loading control. A longer exposure time was used for SOX 9 detection in C3H10T1/2 cells and periosteal cells compared with chondrocytes in order to visualize any remaining protein in the shSOX9 conditions ( $n=2$ independent experiments for $\mathrm{C} 3 \mathrm{H} 10 \mathrm{~T} 1 / 2$ cells, $n=3$ biologically independent samples for periosteal cells, growth plate-derived chondrocytes). I, Quantification of cell viability of C3H10T1/2 cells, periosteal cells and growth plate-derived chondrocytes transduced with shSox 9 or shSCR, after $72 \mathrm{~h}$ of exposure to control, SD or CND medium ( $n=3$ independent experiments for $\mathrm{C} 3 \mathrm{H} 10 \mathrm{~T} 1 / 2$ cells, $n=3$ biologically independent samples for periosteal cells, growth plate-derived chondrocytes). Data are mean \pm s.e.m.; two-way ANOVA with Bonferroni post hoc test $(\mathbf{b}, \mathbf{d}, \mathbf{h}, \mathbf{l})$, two-tailed Student's $t$-test $(\mathbf{c}, \mathbf{j})$. For gel source data, see Supplementary Fig. 1 . 


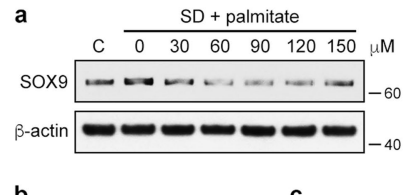

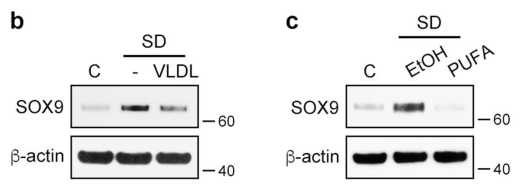
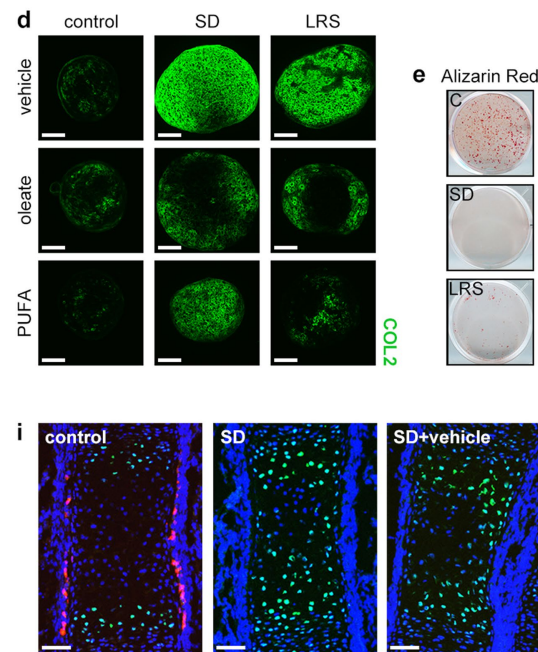

Col1a1-DsRed SOX9
Nuclei

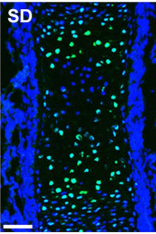

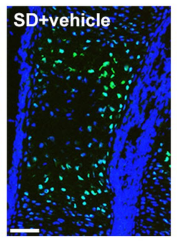
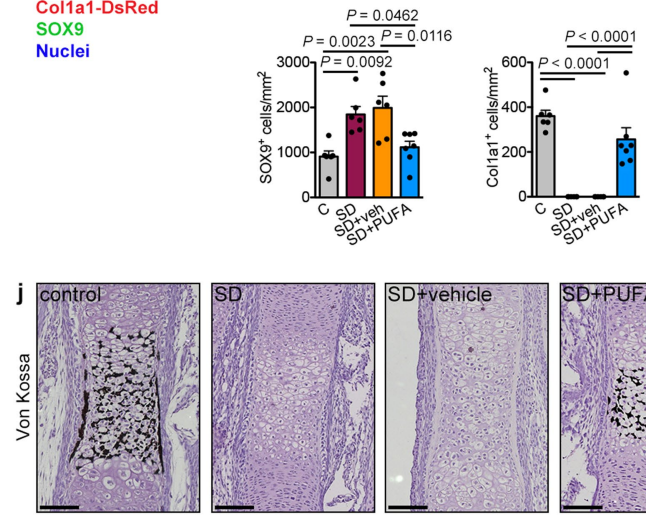

m FL-C16 diffusion

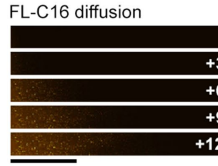

2-NBDG diffusion
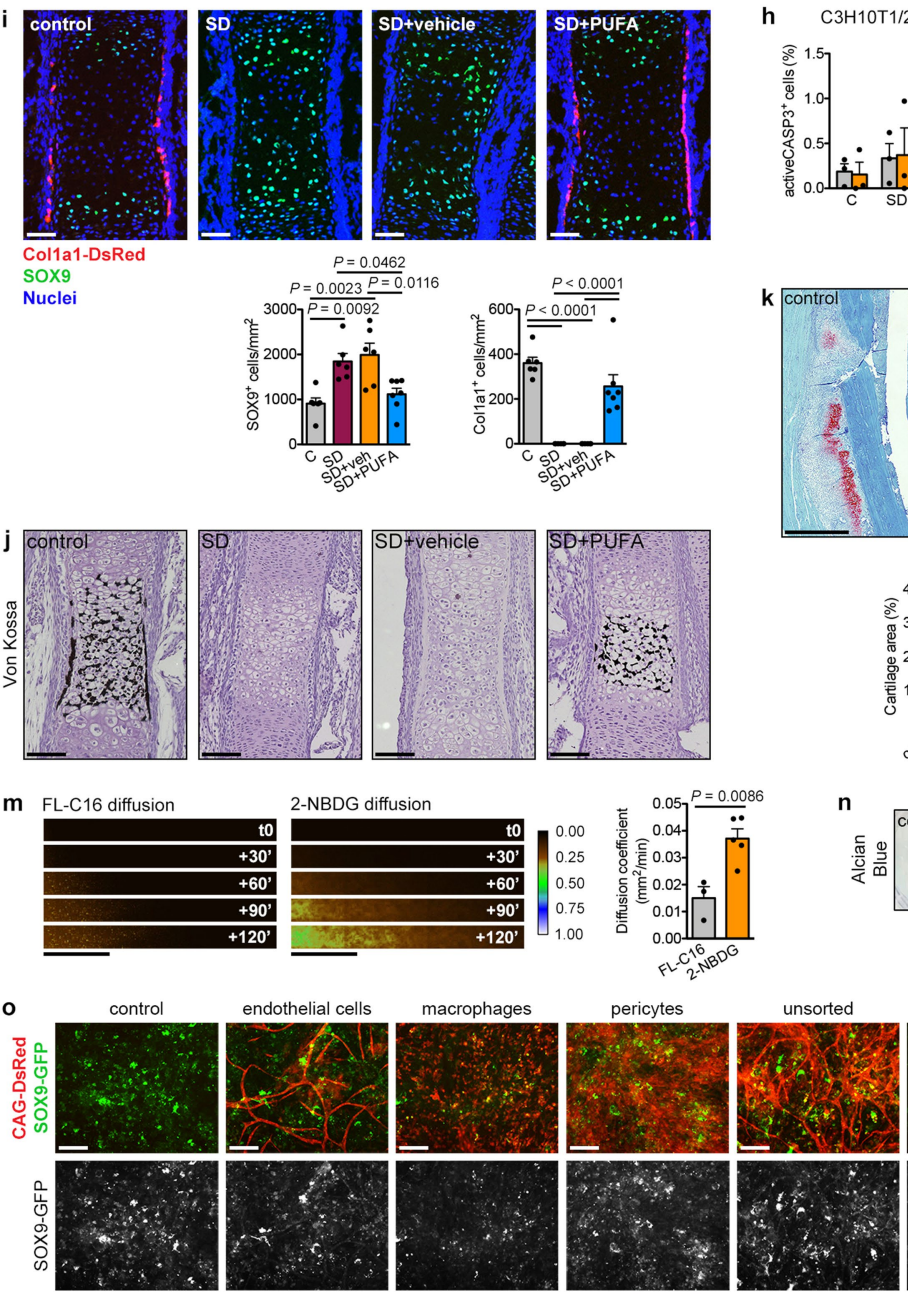
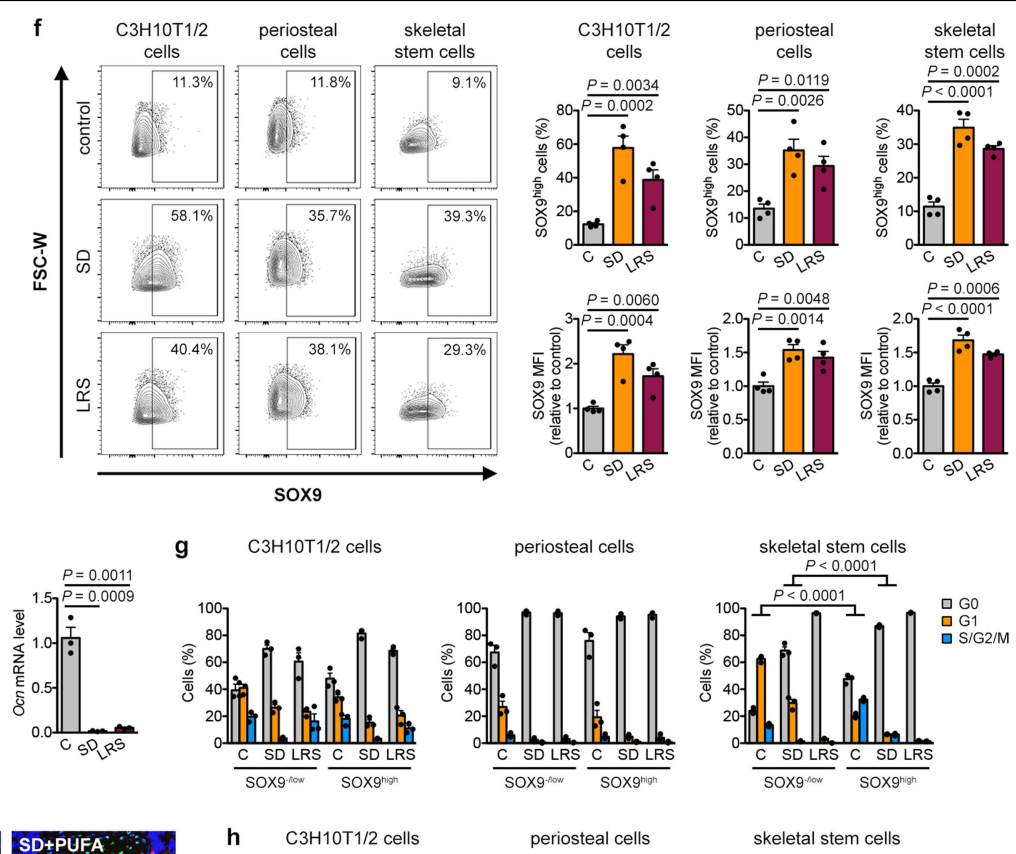

skeletal stem cells
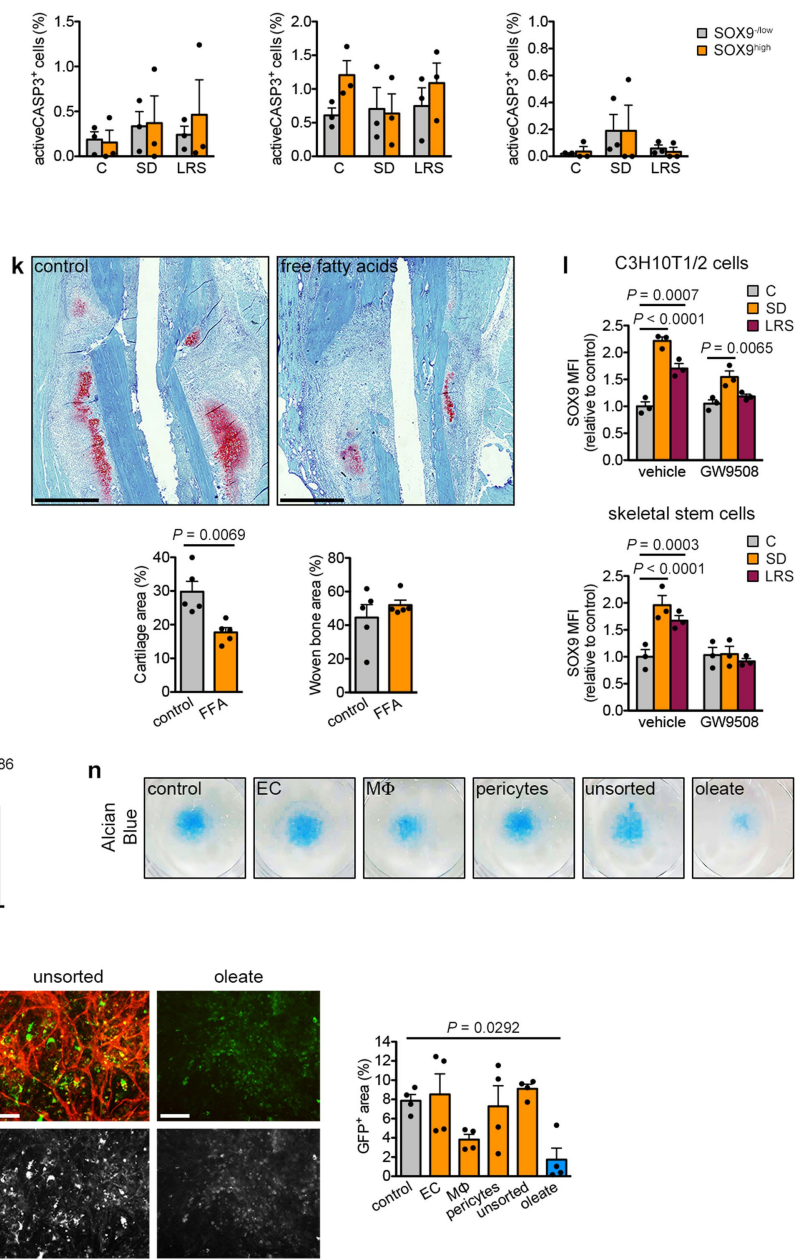

Extended Data Fig. 5 | See next page for caption. 
Extended Data Fig. 5 | Reduced lipid availability favours chondrogenesis over osteogenesis. a-c, Immunoblot detection of total SOX9 in C3H10T1/2 cells exposed for $6 \mathrm{~h}$ to control medium, SD medium or SD medium supplemented with increasing concentrations of palmitate (a), VLDL (b) or PUFA (c). Detection of $\beta$-actin was used as loading control. EtOH was used as a vehicle control in a and $\mathbf{c}$ ( $n=2$ independent experiments). d, Histological visualization (by immunofluorescence for $\mathrm{COL} 2$ ) of chondrogenic differentiation of periosteal cells in pellet cultures in control, SD or LRS medium supplemented with vehicle (EtOH), oleate or PUFA (representative images of $n=2$ independent experiments). Scale bars, $100 \mu \mathrm{m}$. e, Osteogenic differentiation of periosteal cells in control, SD or LRS medium, assessed by visualization of mineral deposits (alizarin red staining) and quantification of $O c n$ mRNA levels (relative to $A c t b, n=3$ biologically independent samples). f, Flow cytometric detection and quantification of the percentage of SOX $9^{\text {high }}$ cells and total SOX9 levels in C3H1OT1/2 cells, periosteal cells and skeletal stem cells exposed for $24 \mathrm{~h}$ to control, SD or LRS medium ( $n=4$ independent experiments for $\mathrm{C} 3 \mathrm{H} 10 \mathrm{~T} 1 / 2$ cells, $n=4$ biologically independent samples for periosteal cells, skeletal stem cells). Gating for $\mathrm{SOX}^{9^{\text {high }}}$ cells was set to have approximately $10 \% \mathrm{SOX} 9^{\text {high }}$ cells in control conditions in each cell type. $\mathbf{g}, \mathbf{h}$, Flow cytometric quantification of cell cycle (g) and apoptosis $(\mathbf{h})$ in SOX ${ }^{\text {low }}$ and SOX $9^{\text {high }}$ subpopulations of C $3 \mathrm{H} 10 \mathrm{~T} 1 / 2$ cells, periosteal cells and skeletal stem cells exposed for $24 \mathrm{~h}$ to control, SD or LRS medium $(n=3$ independent experiments for $\mathrm{C} 3 \mathrm{H} 10 \mathrm{~T} 1 / 2$ cells, $n=3$ biologically independent samples for periosteal cells, skeletal stem cells). i, Histological visualization and quantification of early chondrogenic $\left(\mathrm{SOX}^{+}\right)$and osteogenic (Col1a1DsRed $^{+}$) cells in metatarsals cultured for one week in control medium, SD medium or SD medium supplemented with PUFA or vehicle (EtOH) $(n=6$ biologically independent samples for control, SD and SD + vehicle, $n=7$ biologically independent samples for SD + PUFA). Scale bars, $50 \mu \mathrm{m}$. $\mathbf{j}$, Histological visualization of mineralization by Von Kossa staining in metatarsals cultured for one week in control medium, SD medium or SD medium supplemented with vehicle or PUFA (representative images of $n=6$ biologically independent samples for control, SD and SD + vehicle, $n=7$ biologically independent samples for SD + PUFA). Scale bars, $100 \mu \mathrm{m}$. k, Histological visualization (safranin O staining) and quantification of cartilage and woven bone in the callus at PFD7 of mice treated daily with free fatty acids (FFA; $20 \mu \mathrm{l}$ corn oil) or sham injection (saline) at the fracture site $(n=5$ mice). Scale bars, $500 \mu \mathrm{m}$. I, Flow cytometric quantification of total SOX 9 levels in C3H10T1/2 cells or skeletal stem cells exposed for $24 \mathrm{~h}$ to control, SD or LRS medium supplemented with $100 \mu \mathrm{M} \mathrm{GW} 9508$ or vehicle (DMSO) $(n=3$ independent experiments for $\mathrm{C} 3 \mathrm{H} 10 \mathrm{~T} 1 / 2$ cells, $n=3$ biologically independent samples for skeletal stem cells). $\mathbf{m}$, Visualization and quantification of diffusion of a fluorescent fatty acid (FL-C16) and fluorescent glucose (2-NBDG) in collagen gels seeded with periosteal cells $\left(5 \times 10^{6}\right.$ per $\left.\mathrm{ml}\right)(n=3$ biologically independent samples for FL-C16, $n=5$ biologically independent samples for 2-NBDG). Scale bars, $500 \mu \mathrm{m}$. n, o, Visualization of alcian blue staining (n) and visualization and quantification of Sox 9 expression (o) in micromass cocultures of periosteal cells from Sox9-GFP mice and sorted cell populations from skeletal muscle of CAG-DsRed mice, after nine days in chondrogenic SD medium ( $n=4$ biologically independent samples). Addition of oleate was used as positive control. Scale bars, $100 \mu \mathrm{m}$. EC, endothelial cell, $\mathrm{M} \Phi$, macrophage. Data are mean \pm s.e.m.; one-way ANOVA $(\mathbf{e}, \mathbf{f}, \mathbf{i}, \mathbf{o})$, two-way ANOVA $(\mathbf{h}, \mathbf{l})$ or three-way ANOVA (g) with Bonferroni post hoc test, two-tailed Student's $t$-test $(\mathbf{k}, \mathbf{m})$. For gel source data, see Supplementary Fig.1. 


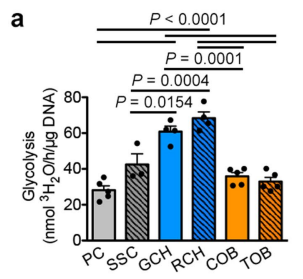

b
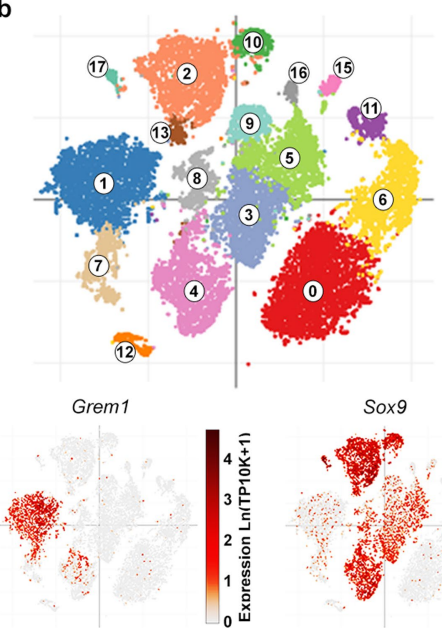

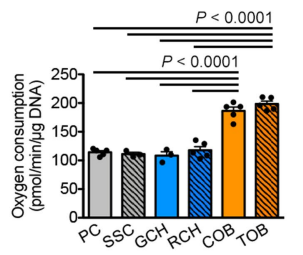

(0) Sinusoidal endothelial cells

(1) Mesenchymal stromal cells

(2) Hypertrophic chondrocytes

(3) Fibroblasts (subcluster 4)

(4) Chondrogenic progenitor cells

(5) Fibroblasts (subcluster 5)

(8) Osteoblasts (subcluster 2)
6) Arteriolar endothelial cells

(7) Osteoblasts (subcluster 1)
(9) Fibroblasts (subcluster 1)

(10) Resting chondrocytes

(11) Arterial endothelial cells

(12) Pericytes

(13) Proliferating chondrocytes

(15) Fibroblast (subcluster 2)

(16) Fibroblast (subcluster 3)

(17) Prehypertrophic chondrocytes
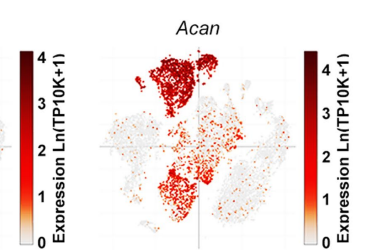

SIc2a1

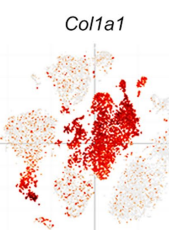

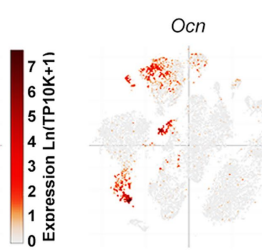

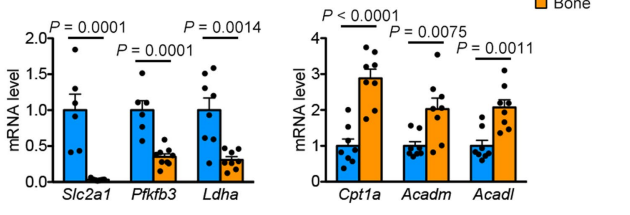

e Red-C12 Nuclei

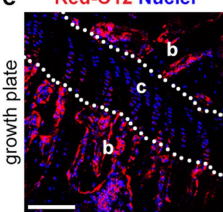

2-NBDG Nuclei
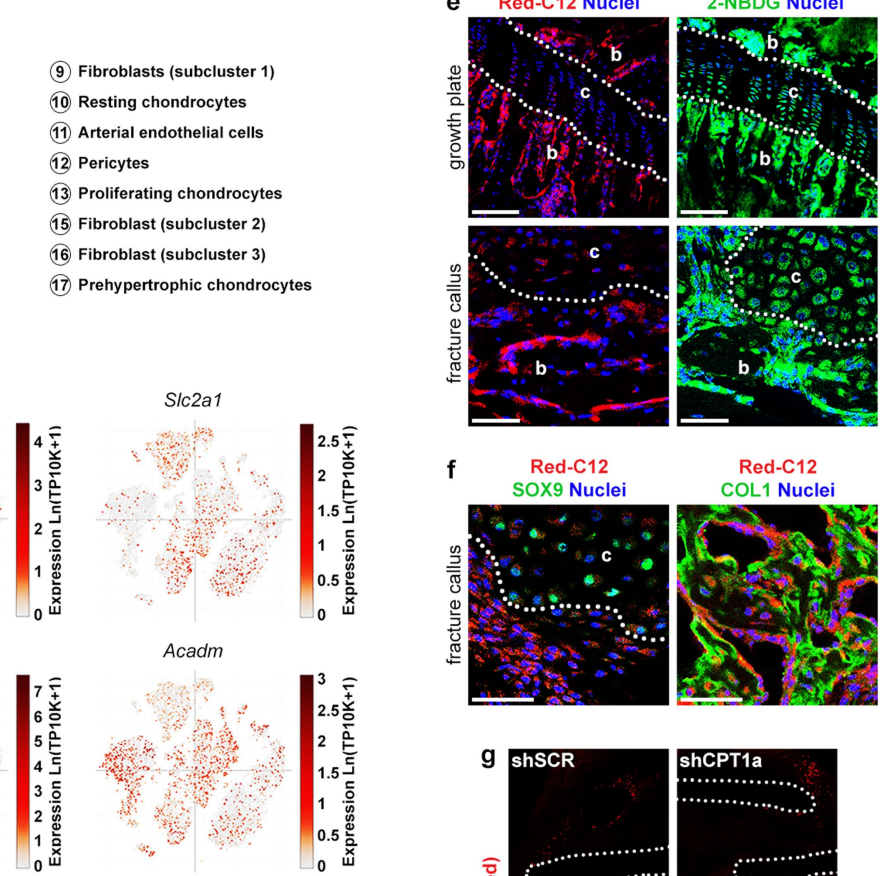

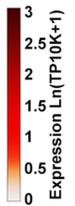
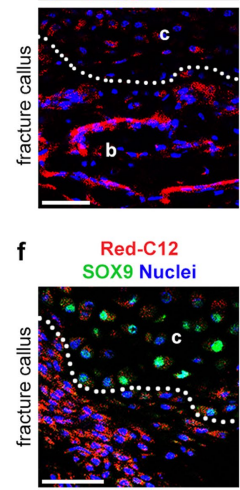

Red-C12
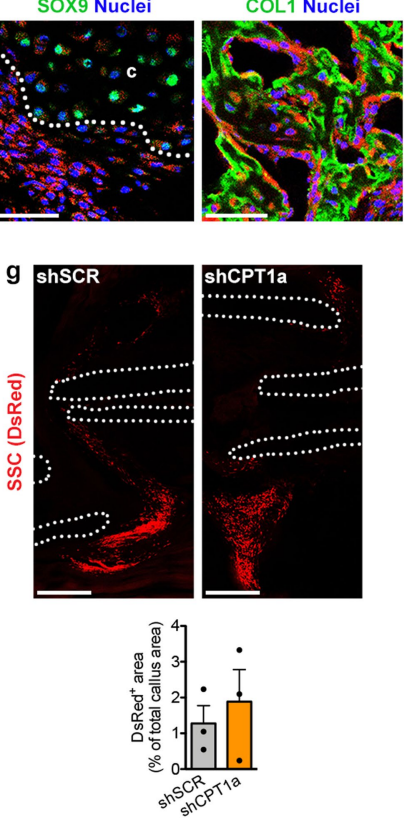

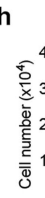

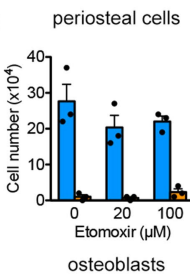

chondrocytes

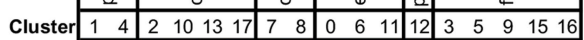

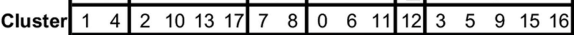
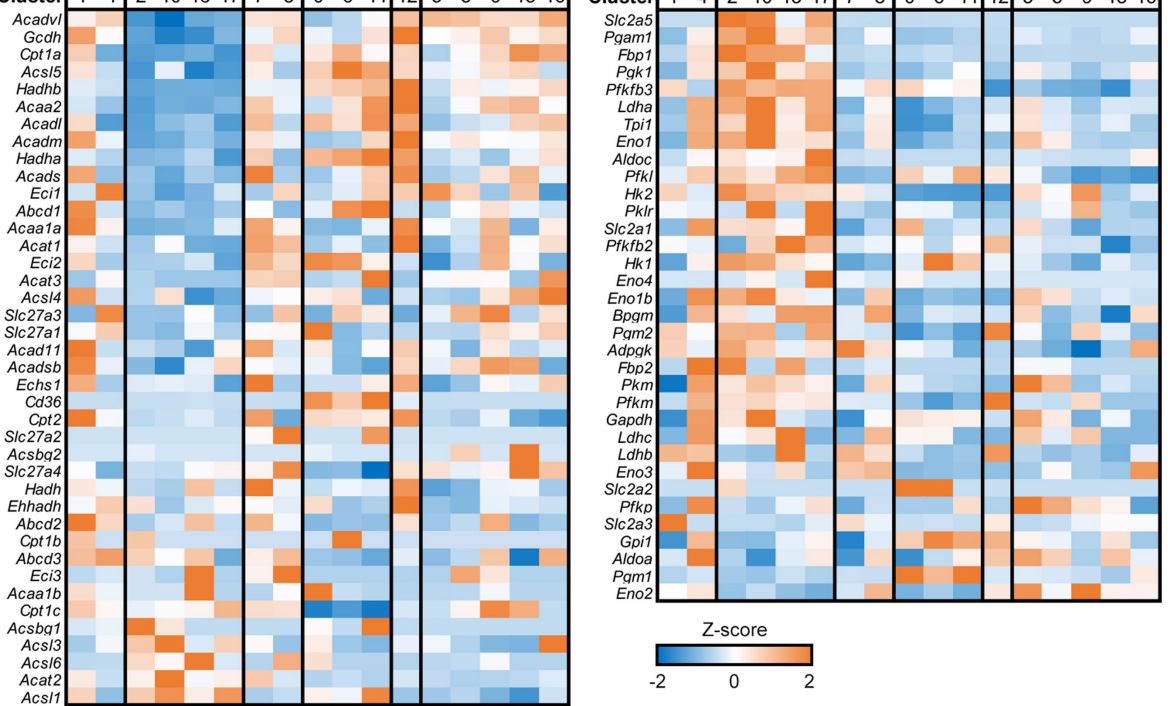

Z-score

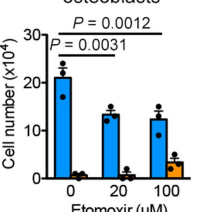

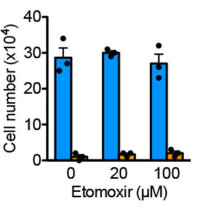

ㄴ Viable

Extended Data Fig. 6 | See next page for caption. 
Extended Data Fig. 6 | Chondrocytes do not depend on FAO. a, Quantification of glycolytic rate, oxygen consumption and palmitate oxidation in periosteal cells (PC, $n=5$ biologically independent samples), skeletal stem cells (SSC, $n=3$ biologically independent samples), growth plate-derived chondrocytes $(\mathrm{GCH}$, $n=3$ biologically independent samples for oxygen consumption, $n=4$ biologically independent samples for glycolysis and palmitate oxidation), rib chondrocytes $(\mathrm{RCH}, n=5$ biologically independent samples for oxygen consumption, $n=4$ biologically independent samples for glycolysis and palmitate oxidation), calvarial osteoblasts ( $\mathrm{COB}, n=5$ biologically independent samples) and trabecular osteoblasts (TOB, $n=5$ biologically independent samples). $\mathbf{b}, t$-Distributed stochastic neighbour embedding ( $t$-SNE) plot of 20,896 non-haematopoietic cells (mixed bone and bone marrow fractions, $n=6$ mice) based on single-cell RNA-seq data, annotated post hoc and coloured by clustering (top) or by expression (In(TP10K)) of selected genes (bottom). c, Expression (row-wide $Z$ score of In of average TP10K; single-cell RNA-seq) of FAO- and glycolysis-related genes (rows) in the cells of each cluster (columns). d, RT-qPCR analysis of genes involved in glycolysis (Glut1 (also known as Slc2a1), $P f k f b 3$ and $L d h a ; n=6$ independent samples for Glut 1 and $P f k f b 3$ in cartilage, $n=9$ independent samples for Glut 1 and $P f k f b 3$ in bone, $n=8$ independent samples for Ldha) and FAO (Cpt1a, Acadm and Acadl; $n=8$ independent samples) in mouse growth plate cartilage and cortical bone biopsies (relative to Actb).e, Analysis of adjacent histological sections of a growth plate and fracture callus (PFD7) of mice injected intravenously with a fluorescent fatty acid (Red-C12) or glucose (2-NBDG) (representative images of $n=3 \mathrm{mice})$. Scale bars, $100 \mu \mathrm{m}$ in growth plate images, $50 \mu \mathrm{m}$ in fracture callus images.f, Immunofluorescence analysis of a fracture callus (PFD7) of a mouse injected intravenously with a fluorescent fatty acid (Red-C12) and stained for SOX9 (left; cartilage area shown) or COL1 (right; trabecular bone area shown) (representative images of $n=3$ mice). Scale bars, $50 \mu \mathrm{m}$. g, Histological visualization and quantification at PFD7 of CAG-DsRed ${ }^{+}$skeletal stem cells (SSC), transduced with shCpt1a or shScr and transplanted at the fracture site on PFD0 ( $n=3$ mice). Dotted lines delineate cortical bone ends. $\mathbf{h}$, Quantification of number of live and dead cells in cultures of periosteal cells, growth platederived chondrocytes and calvarial osteoblasts after $48 \mathrm{~h}$ of exposure to etomoxir ( $n=3$ biologically independent samples). Data are mean \pm s.e.m.; oneway (a) or two-way (h) ANOVA with Bonferroni post hoc test, two-tailed Student's $t$-test $(\mathbf{d}, \mathbf{g})$. 

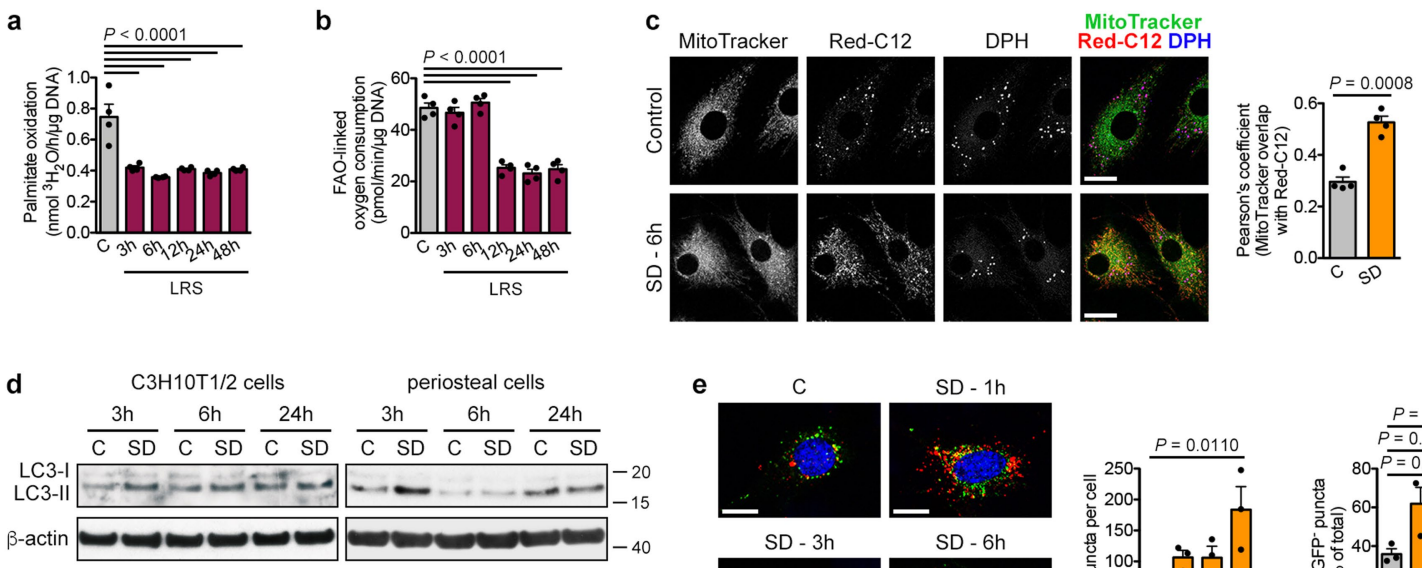
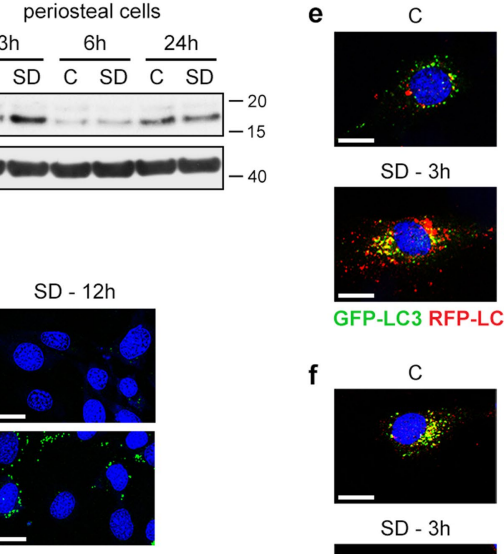

$S D-3 h$

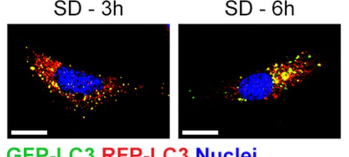

GFP-LC3 RFP-LC3 Nuclei
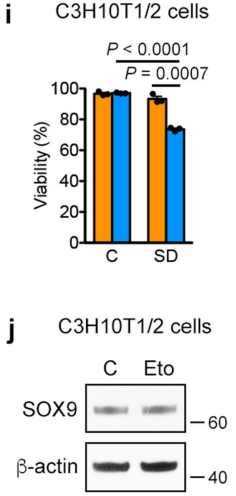

Extended Data Fig. 7 | Changes in FAO and autophagy after lipid deprivation. a, Oxidation of extracellularly added palmitate by periosteal cells in control medium or at different times in LRS medium $(n=4$ biologically independent samples). b, Quantification of FAO-linked OCR in periosteal cells in control medium or at different times in LRS medium ( $n=4$ biologically independent samples).c, Confocal microscopy of periosteal cells labelled with Red-C12 (fluorescent fatty acid, red) and stained with MitoTracker (mitochondria, green) and DPH (lipid droplets, blue) shows increased colocalization (as quantified by Pearson's correlation coefficient) of MitoTracker and Red-C12 after exposure of cells for $6 \mathrm{~h}$ to SD medium $(n=4$ biologically independent samples). Scale bars, $20 \mu \mathrm{m}$. d, Immunoblot detection of LC3 in total cell protein extracts of $\mathrm{C} 3 \mathrm{H} 10 \mathrm{~T} 1 / 2$ cells and periosteal cells exposed for different times to control or SD medium, with $\beta$-actin as loading control. Note increased conversion of LC3-I to LC3-II at early time points, indicative of activation of autophagy ( $n=2$ independent experiments).e,f, Confocal microscopy of C3H10T1/2 cells (e; $n=3$ independent experiments) or periosteal cells (f; $n=3$ biologically independent samples), expressing an RFP-GFP-LC3 tandem construct, shows activation of autophagy with time upon serum deprivation, evidenced by increased total number of LC3 puncta per cell and higher percentage of $\mathrm{RFP}^{+} \mathrm{GFP}^{-}$puncta. Scale bars, $20 \mu \mathrm{m}$. g, Confocal microscopybased visualization (top) and quantification (bottom) of C $3 \mathrm{H} 10 \mathrm{~T} 1 / 2$ cells, stained with the neutral lipid dye DPH to reveal lipid-droplet dynamics at different time points after SD. Cells were transduced with shAtg5 to inhibit

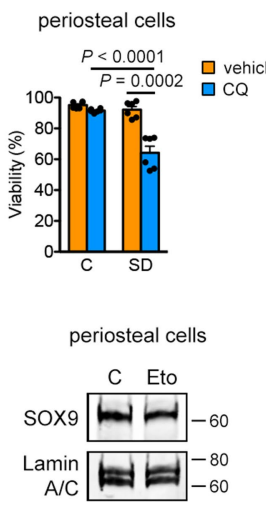

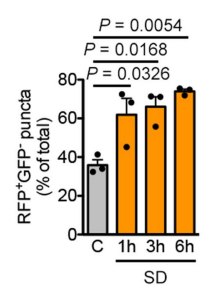

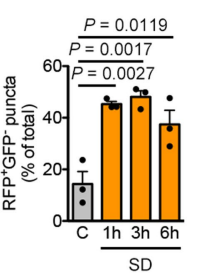

k $\quad$ ShSCR $\operatorname{shSOX9}$
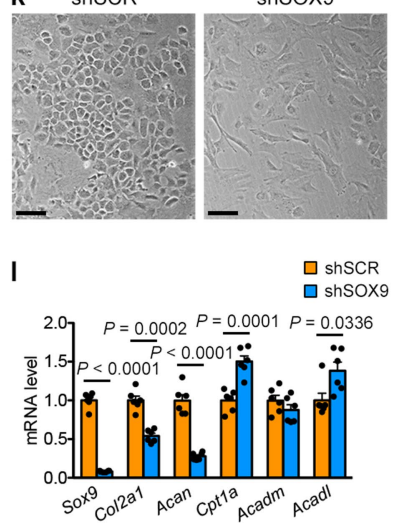

autophagy or shScr as a control ( $n=6$ independent experiments). Scale bars, $20 \mu \mathrm{m}$. h, Quantification of FAO-linked OCR in periosteal cells in control medium or at different times after serum deprivation, treated with $10 \mu \mathrm{M}$ chloroquine (CQ) or vehicle ( $n=3$ biologically independent samples). i, Quantification of cell viability of $\mathrm{C} 3 \mathrm{H} 10 \mathrm{~T} 1 / 2$ cells and periosteal cells after $72 \mathrm{~h}$ of exposure to control or SD medium in the presence or absence of $50 \mu \mathrm{M}$ (C3H10T1/2 cells) or $10 \mu \mathrm{M}$ (periosteal cells) CQ $(n=3$ independent experiments for $\mathrm{C} 3 \mathrm{H} 10 \mathrm{~T} 1 / 2$ cells, $n=3$ biologically independent samples for periosteal cells).j, Immunoblot detection of total SOX9 in C3H10T1/2 cells and nuclear SOX9 in periosteal cells exposed for $6 \mathrm{~h}$ (C3H10T1/ 2 cells) or $24 \mathrm{~h}$ (periosteal cells) to control medium (with DMSO as vehicle control) or medium supplemented with $100 \mu \mathrm{M}$ etomoxir (Eto), with $\beta$-actin or lamin A/C as loading control ( $n=2$ independent experiments for $\mathrm{C} 3 \mathrm{H} 10 \mathrm{~T} 1 / 2$ cells, $n=3$ biologically independent samples for periosteal cells). k, Cell morphology of growth platederived chondrocytes transduced with shSox 9 or shScr (representative images of six biologically independent samples). Scale bar, $100 \mu \mathrm{M} .1, \mathrm{RT}-\mathrm{qPCR}$ analysis of genes involved in chondrogenesis (Sox9, Col2a1 and Acan) and FAO (Cpt1a, Acadm and Acadl) in growth plate-derived chondrocytes transduced with shSox 9 or shScr (relative to $\operatorname{shScr}, n=6$ biologically independent samples). Data are mean \pm s.e.m.; one-way $\operatorname{ANOVA}(\mathbf{a}, \mathbf{b}, \mathbf{e}, \mathbf{f})$ or two-way $\operatorname{ANOVA}(\mathbf{g}-\mathbf{i})$ with Bonferroni post hoc test, two-tailed Student's $t$-test (c, I). For gel source data, see Supplementary Fig. 1. 

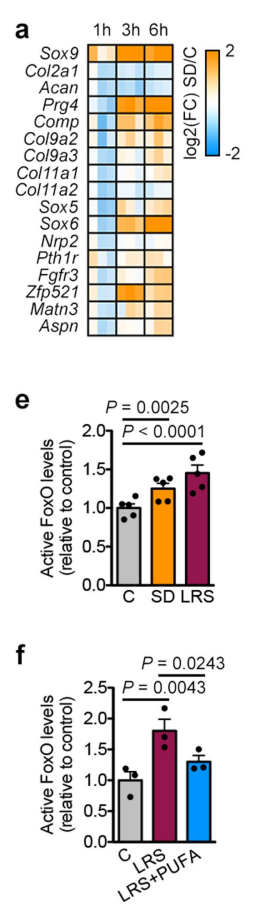

g
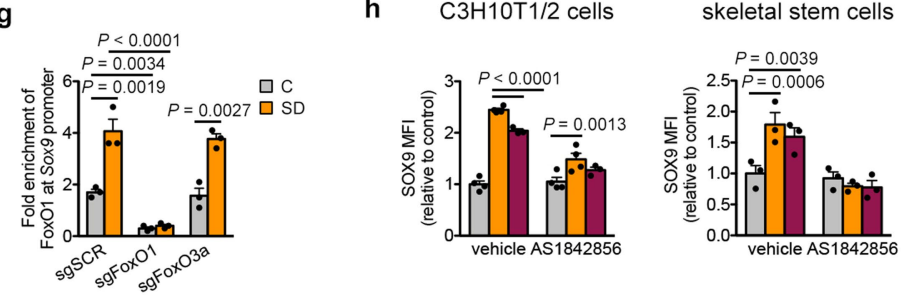

$\square C$
$\square S D$
$\square$ LRS
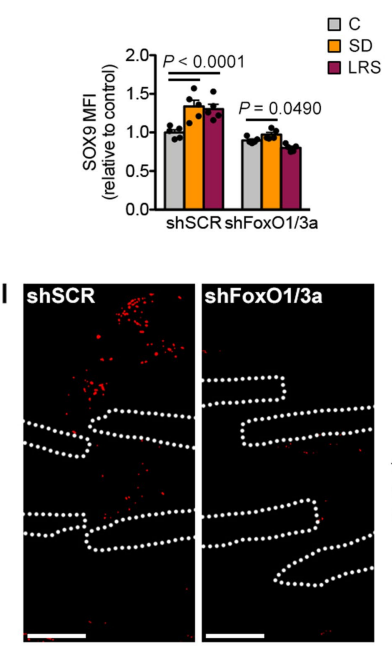

SSC (DsRed)

j skeletal stem cells SD

k
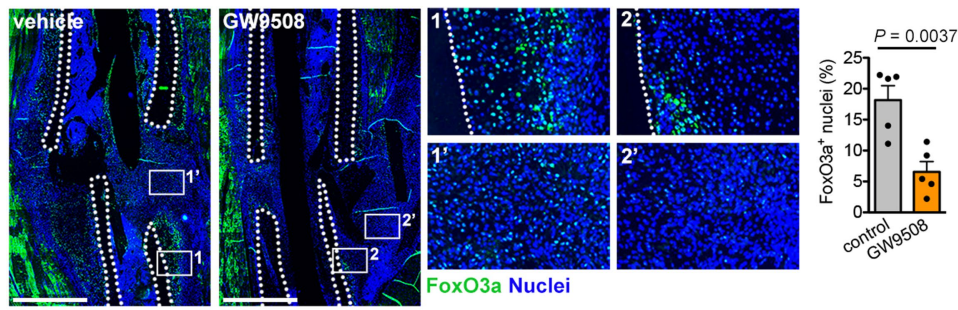
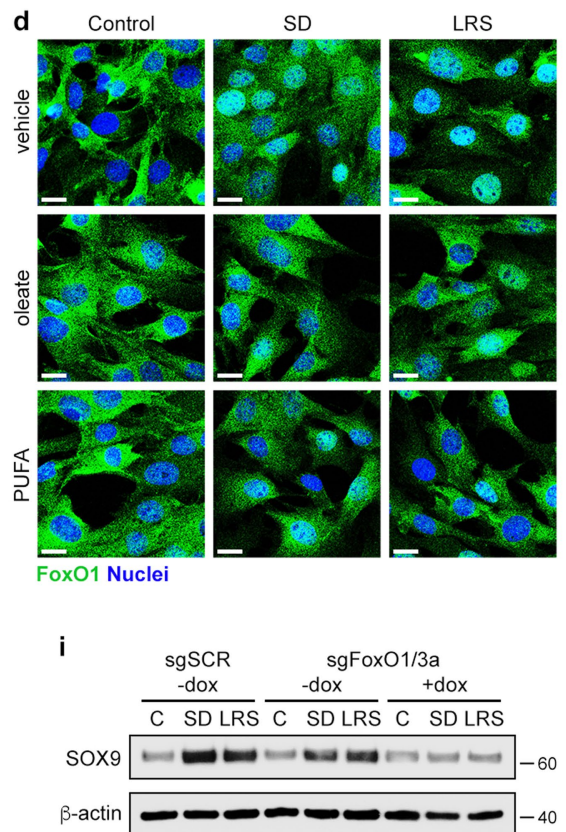

Extended Data Fig. 8 | Lipids regulate SOX9 through FOXO signalling. a, Heat map showing differential expression of cartilage-related genes in C3H10T1/2 cells exposed for different times to SD versus control medium, as determined by RNA-seq ( $n=3$ replicates). b, Volcano plot showing significantly enriched and depleted mRNAs in C3H10T1/2 cells exposed for 3 or $6 \mathrm{~h}$ to SD versus control medium, as determined by RNA-seq ( $n=3$ replicates). c, Top 10 most significantly enriched transcription factor motifs with normalized enrichment scores (NES) in C3H10T1/ 2 cells exposed for $3 \mathrm{~h}$ (left) or $6 \mathrm{~h}$ (right) to SD versus control medium, as determined by $\mathrm{i}$-cisTarget analysis on the 100 mostsignificantly increased mRNAs ( $n=3$ replicates). Motif shown on top is the Hmgal motif for $3 \mathrm{~h}$ and the Atf 4 motif for $6 \mathrm{~h}$. d, Confocal microscopy of C3H10T1/2 cells stained for FOXO1 after exposure of cells for $3 \mathrm{~h}$ to SD or LRS medium in the presence of vehicle $(\mathrm{EtOH})$, oleate $(60 \mu \mathrm{M})$ or PUFA

(representative images of two independent experiments). Scale bars, $20 \mu \mathrm{m}$. e, Nuclear FOXO activity in C3H10T1/2 cells exposed for $3 \mathrm{~h}$ to control, SD or LRS medium ( $n=5$ independent experiments).f, Nuclear FOXO activity in skeletal stem cells exposed for $3 \mathrm{~h}$ to control medium, LRS medium or LRS medium supplemented with PUFA ( $n=3$ biologically independent samples). EtOH was used as vehicle control.g, Occupancy of FOXO1 at the Sox 9 promoter of Cas9-expressing C3H10T1/2 cells transduced with sgFoxo1, sgFoxo3a or sgScr, exposed for $3 \mathrm{~h}$ to control or SD medium, as determined by ChIP-qPCR ( $n=3$ independent experiments). $\mathbf{h}$, Flow cytometric quantification of total
SOX9 levels in C3H10T1/2 cells ( $n=4$ independent experiments for control and serum deprivation, $n=3$ independent experiments for LRS) and skeletal stem cells ( $n=3$ biologically independent samples) exposed for $24 \mathrm{~h}$ to control, SD or LRS medium supplemented with $1 \mu \mathrm{M}$ AS1842856 or vehicle (DMSO). i, Immunoblot detection of total SOX9 in Cas9-expressing C3H10T1/2 cells transduced with inducible sgFoxo1 and sgFoxo $3 a(\mathrm{sg} F o x o 1 / 3 a)$ or with sgScr, exposed for $6 \mathrm{~h}$ to control, SD or LRS medium in the presence or absence of doxycycline (dox; $\left.250 \mathrm{ng} \mathrm{ml}^{-1}\right)$, with $\beta$-actin as loading control $(n=2$ independent experiments).j, Flow cytometric quantification of total SOX 9 levels in skeletal stem cells transduced with shFoxo1 and $\operatorname{shFoxo3a}(\operatorname{sh} F o x o 1 / 3 a)$ or with shScr, exposed for $24 \mathrm{~h}$ to control, SD or LRS medium ( $n=5$ biologically independent samples). $\mathbf{k}$, Histological visualization and quantification of FOXO3a-expressing cells in the fracture callus at PFD7 of mice treated daily with GW9508 $(10 \mathrm{nmol})$ or vehicle $(0.2 \%$ DMSO in saline) at the fracture site ( $n=5$ mice). Scale bars, $500 \mu \mathrm{m}$. Dotted lines delineate cortical bone ends. 1, Histological visualization and quantification in the fracture callus at PFD7 of CAG-DsRed ${ }^{+}$skeletal stem cells (SSC), transduced with shFoxo1/3a or shScr and transplanted at the fracture site on PFD0 ( $n=5$ mice). Dotted lines delineate cortical bone ends. Data are mean \pm s.e.m.; one-way ANOVA (e,f), two-way $\operatorname{ANOVA}(\mathbf{g}, \mathbf{h}, \mathbf{j})$ with Bonferroni post hoc test, two-tailed Student's $t$-test $(\mathbf{k}, \mathbf{l})$. For gel source data, see Supplementary Fig. 1 . 
a
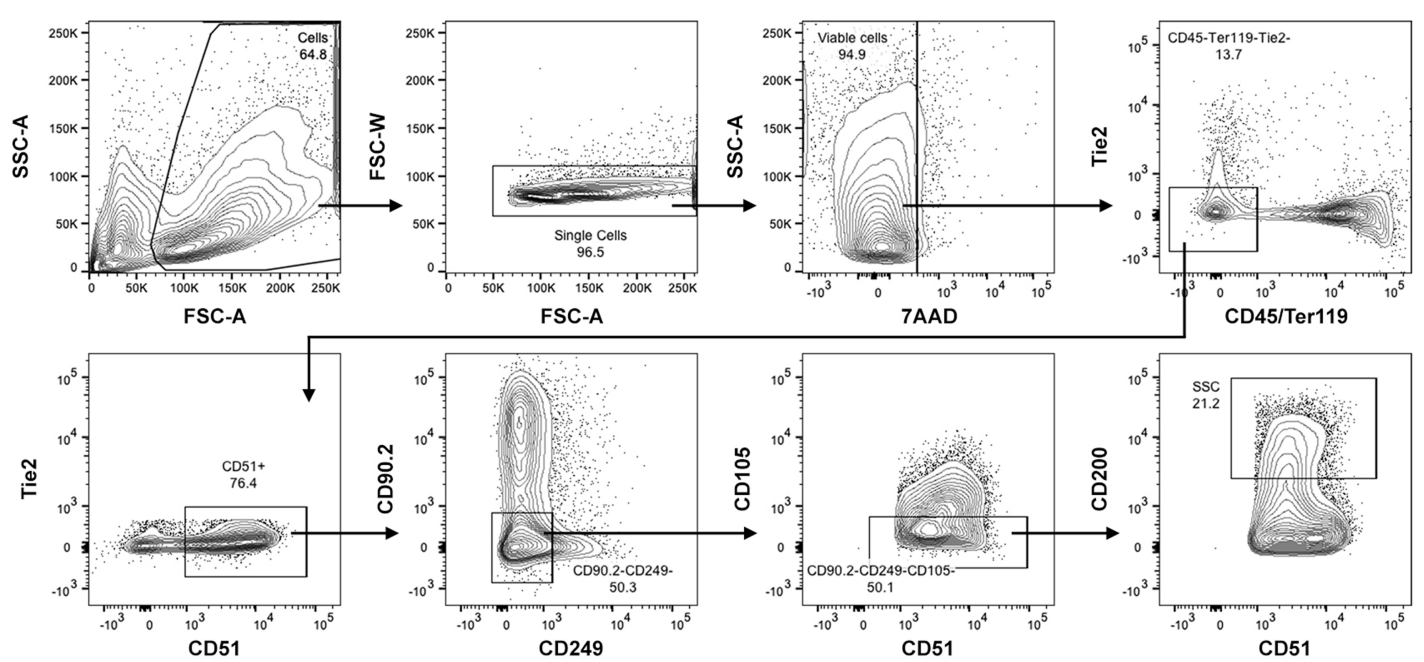

b
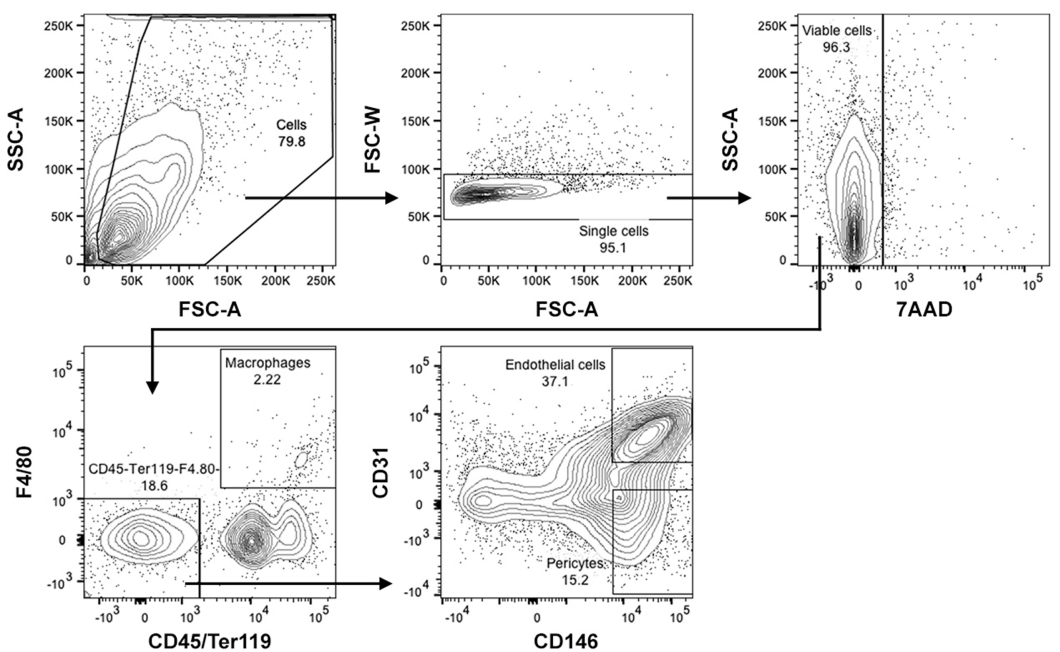

Extended Data Fig. 9 | Flow cytometry gating for cell sorting. a, Contour plots showing the gating strategy for the identification and isolation of skeletal stem cells from long bones of newborn mice. b, Contour plots showing the

gating strategy for the identification and isolation of macrophages, endothelial cells and pericytes from skeletal muscle of adult mice. 


\section{Reporting Summary}

Nature Research wishes to improve the reproducibility of the work that we publish. This form provides structure for consistency and transparency in reporting. For further information on Nature Research policies, see Authors \& Referees and the Editorial Policy Checklist.

\section{Statistics}

For all statistical analyses, confirm that the following items are present in the figure legend, table legend, main text, or Methods section.

n/a Confirmed

$\square$ \. $\square$ The exact sample size $(n)$ for each experimental group/condition, given as a discrete number and unit of measurement

$\square$ \ A statement on whether measurements were taken from distinct samples or whether the same sample was measured repeatedly

$\square$ The statistical test(s) used AND whether they are one- or two-sided

$\square$ Only common tests should be described solely by name; describe more complex techniques in the Methods section.

Х $\square$ A description of all covariates tested

$\square$ \A description of any assumptions or corrections, such as tests of normality and adjustment for multiple comparisons

$\square$ A full description of the statistical parameters including central tendency (e.g. means) or other basic estimates (e.g. regression coefficient)

$\square$ AND variation (e.g. standard deviation) or associated estimates of uncertainty (e.g. confidence intervals)

$\square$ For null hypothesis testing, the test statistic (e.g. $F, t, r$ ) with confidence intervals, effect sizes, degrees of freedom and $P$ value noted

$\square$ Give P values as exact values whenever suitable.

Х $\square$ For Bayesian analysis, information on the choice of priors and Markov chain Monte Carlo settings

Х $\square$ For hierarchical and complex designs, identification of the appropriate level for tests and full reporting of outcomes

$\square$ Estimates of effect sizes (e.g. Cohen's $d$, Pearson's $r$ ), indicating how they were calculated

Our web collection on statistics for biologists contains articles on many of the points above.

\section{Software and code}

Policy information about availability of computer code

Data collection $\quad$ QRT-PCR: StepOne Real-Time PCR software 2.3

Flow cytometry: BD FACSDiva 8.0

Seahorse flux analyzer: XF Reader 1.8.1.1

scintillation counting: QuantaSmart TM 4.0 Perkin Elmer

Imaging: Zeiss Zen 2.5, Zeiss AxioVision 4.9.1

Computer Modeling: MatLab R2012a/R2013a

Data analysis

CT: CT Analyzer 1.16.4.1, CT Vol 2.3.2.0, MeVisLab 2.6.2

Flow cytometry: FlowJo 10.5.3

Statistics: GraphPad Prism 8.1.2

Graphs: GraphPad Prism 5.0

Image analysis: ImageJ/FIJI 2.0.0, CellProfiler 3.1.8

Image preparation: Photoshop CS5

RNAseq/scRNAseq analysis: TopHat 2.1.1, R statistical software 3.5.1, EdgeR 3.20.9, i-cisTarget

For manuscripts utilizing custom algorithms or software that are central to the research but not yet described in published literature, software must be made available to editors/reviewers. We strongly encourage code deposition in a community repository (e.g. GitHub). See the Nature Research guidelines for submitting code \& software for further information. 
Policy information about availability of data

All manuscripts must include a data availability statement. This statement should provide the following information, where applicable:

- Accession codes, unique identifiers, or web links for publicly available datasets

- A list of figures that have associated raw data

- A description of any restrictions on data availability

The bulk mRNA sequencing data that support the findings of this study have been deposited in ArrayExpress with the accession number E-MTAB-7564 (http:// www.ebi.ac.uk/arrayexpress/experiments/E-MTAB-7564). The single cell RNA sequencing data were generated previously and are deposited in GEO (GSE128423, https://www.ncbi.nlm.nih.gov/geo/query/acc.cgi?acc=GSE128423). A portal for exploring the entire atlas is available (https://portals.broadinstitute.org/single_cell/ study/mouse-bone-marrow-stroma-in-homeostasis). All other data supporting the findings of this study are available within the paper.

Figures 2,4 and Extended Data Figures 4,5,7,8 have associated raw data, provided as Supplemental Information Figure 1 for uncropped blot pictures

\section{Field-specific reporting}

Please select the one below that is the best fit for your research. If you are not sure, read the appropriate sections before making your selection. \Life sciences $\square$ Behavioural \& social sciences Ecological, evolutionary \& environmental sciences

For a reference copy of the document with all sections, see nature.com/documents/nr-reporting-summary-flat.pdf

\section{Life sciences study design}

All studies must disclose on these points even when the disclosure is negative.

Sample size

No statistical tests were used to pre-determine sample size, but sample size was chosen based on previous experiments and comparable studies in literature. Sample size for each experiment is indicated in the legend.

Data exclusions No data was excluded.

Replication In the studies performed in cell lines in culture, all experiments were independently repeated at least three times. Experiments using primary cells were performed with at least three biological replicates. Western Blots were independently repeated at least twice. All attempts at replication were successful

Randomization Mice for experiments were randomly allocated to groups.

\begin{tabular}{l|l} 
Blinding & Blinding was widely used in the study. Data collection and analysis, such as immunostaining, qRT-PCR, and Western blot were frequently
\end{tabular} performed by participants other than the experiment designer. During these data collection and analysis steps, all participants were routinely blinded to group allocation.

\section{Reporting for specific materials, systems and methods}

We require information from authors about some types of materials, experimental systems and methods used in many studies. Here, indicate whether each material, system or method listed is relevant to your study. If you are not sure if a list item applies to your research, read the appropriate section before selecting a response.

Materials \& experimental systems

\begin{tabular}{l|l}
\hline$n / a$ & Involved in the study \\
\hline & $\bigotimes$ Antibodies \\
$\square$ & $\square$ Eukaryotic cell lines \\
$\square$ & $\square$ Animals and other organisms \\
$\square$ & $\square$ Human research participants \\
$\square$ Clinical data
\end{tabular}

\begin{tabular}{l|l}
\multicolumn{2}{l}{ Methods } \\
\hline n/a & Involved in the study \\
$\square$ & $\square$ ChIP-seq \\
$\square$ & $\bigotimes$ Flow cytometry \\
$\square$ & $\square$ MRI-based neuroimaging
\end{tabular}

\section{Antibodies}

Antibodies used 
APC anti-mouse CD105: BioLegend, \#120414, clone MJ7/18, lot B204640, 1/200 dilution

Pacific Blue anti-mouse CD105: BioLegend, \#120412, clone MJ7/18, lot B245562, 1/200 dilution

APC anti-mouse CD90.2: BioLegend, \#105312, clone 30-H12, lot B208842, 1/200 dilution

FITC anti-mouse Ly-51 (6C3): BioLegend, \#108305, clone 6C3, lot B218198, 1/200 dilution

PerCP-eFluor 710 anti-mouse CD200: eBioscience, \#46-5200-82, clone OX90, lot 4298111, 1/200 dilution

Biotin anti-mouse CD51: BD Pharmingen, \#551380, clone RMV-7, lot 3301919, 1/200 dilution

PE anti-Ki67: BD Pharmingen, \#556027, clone B56, lot 5113743, 1/10 dilution

PE anti-active Caspase 3: BD Pharmingen, \#550821, clone C92-605, lot 25660, 1/100 dilution

AlexaFluor 647 anti-SOX9: Cell Signaling Technology, \#71273, clone D8G8H, lot 1, 1/100 dilution

APC anti-mouse CD146: BioLegend, \#134712, clone ME-9F1, lot B268897, 1/200 dilution

Pacific Blue anti-mouse F4/80: BioLegend, \#123124, clone BM8, lot B217178, 1/200 dilution

PerCP/Cy5.5 anti-mouse CD31: BioLegend, \#102522, clone MEC13.3, lot 4052815, 1/200 dilution

Primary antibodies for immunohistochemical analysis:

BrdU: Bio-Rad, \#OBT0030, clone BU 1/75-ICR1, Rat, lot 0512, 1/500 dilution

CD31: BD Biosciences, 550274, clone MEC 13.3, Rat, lot 7292994, 1/50 dilution

type I collagen: Novus Biologicals, clone NB600-408, polyclonal, rabbit, lot 40267, 1/100 dilution

type II collagen: Merck, MAB8887, monoclonal, mouse, clone 6B3, lot 2933390, 1/200 dilution

SOX9: Novus Biologicals, NBP1-85551, polyclonal, rabbit, lot B113838, 1/200 dilution

CPT1a: Cell Signaling Technology, \#12252, clone D3B3, rabbit, lot 1, 1/50 dilution

GLUT1: Cell Signaling Technology, \#12939, clone D3J3A, rabbit, lot 1, 1/100 dilution

FoxO3a: Cell Signaling Technology, \#2497, clone 75D8, rabbit, lot 5, 1/100 dilution

Secondary antibodies for immunohistochemical analysis:

Biotin anti-mouse: Dako, \#E0433, goat, lot 00062137

Fluorescein anti-mouse: Sigma-Aldrich, \#F0257, goat, lot SLBV6490

Biotin anti-rat: BD Biosciences, \#559286, goat, lot 6321784

Biotin anti-rat: Dako, \#E0468, rabbit, lot 00043220

Biotin anti-rabbit: Dako, \#E0432, goat, lot 20027287

Cy3 anti-rabbit: Jackson ImmunoResearch, \#111-165-003, goat, lot 84241

AlexaFluor 546 anti-rabbit: Invitrogen, \#A-11010, goat, lot 1904467

AlexaFluor 488 anti-rabbit: Invitrogen; \#A-11034, goat, lot 1937195

Primary antibodies for immunocytochemical analysis:

FoxO1: Cell Signaling Technology, \#2880, clone C29H4, rabbit, lot 11, 1/100 dilution

FoxO3a: Cell Signaling Technology, \#2497, clone 75D8, rabbit, lot 5, 1/100 dilution

Secondary antibodies for immunocytochemical analysis:

AlexaFluor 488 anti-rabbit: Invitrogen; \#A-11034, goat, lot 1937195

Primary antibodies for Western Blot:

SOX9: Novus Biologicals, NBP1-85551, polyclonal, rabbit, lot B113838, 1/2000 dilution

FoxO1: Cell Signaling Technology, \#2880, clone C29H4, rabbit, lot 11, 1/1000 dilution

FoxO3a: Cell Signaling Technology, \#2497, clone 75D8, rabbit, lot 5, 1/1000 dilution

LC3B: Cell Signaling Technology, \#3868, clone D11, rabbit, lot 11, 1/500 dilution

B-actin: Sigma-Aldrich, \#A5441, clone AC-15, mouse, lot 026M, 1/10000 dilution

Lamin A/C: Santa Cruz Biotechnology, \#sc-376248, clone E-1, mouse, lot C1412, 1/5000 dilution

Secondary antibodies for Western blot analysis:

HRP anti-mouse: Dako, \#P0161, rabbit, lot 00095192

HRP anti-rabbit: Dako, \#P0448, goat, lot 00094764

Primary antibodies for ChIP-qPCR:

FoxO1: Abcam, \#ab39670, rabbit, lot GR3192176-1, 1/250 dilution

FoxO3a: Abcam, \#ab12162, rabbit, lot GR226465-14, 1/250 dilution

All antibodies were obtained from indicated commercial vendors with ensured quality. In addition, all the antibodies have been used in multiple experiments to detect intended proteins in control samples with expected molecular weight to validate their effectiveness in our study

Antibodies for flow cytometry:

PE/Cy7 anti-mouse CD45: BioLegend, \#103114, clone 30-F11

RRID: AB_312979

Validated by the manufacturer by flow cytometry on C57BL/6 mouse splenocytes, 46 citations

PE/Cy7 anti-mouse TER-119: BioLegend, \#116222, clone TER-119

RRID: AB_2281408

Validated by the manufacturer by flow cytometry on C57BL/ 6 mouse bone marrow cells, 6 citations

APC anti-mouse CD202b (Tie-2, CD202): BioLegend, \#124010, clone TEK4

RRID: AB 10897106

Validated by the manufacturer by flow cytometry on bEnd. 3 mouse endothelial cells, 3 citations

PE anti-mouse CD202b (Tie-2, CD202): BioLegend, \#124008, clone TEK4 
RRID: AB 2287338

Validated by the manufacturer by flow cytometry on bEnd. 3 mouse endothelial cells, 5 citations

APC anti-mouse CD105: BioLegend, \#120414, clone MJ7/18

RRID: AB 2277914

Validated by the manufacturer by flow cytometry on bEnd. 3 mouse endothelial cells, 5 citations

Pacific Blue anti-mouse CD105: BioLegend, \#120412, clone MJ7/18

RRID: AB 209889

Validated by the manufacturer by flow cytometry on bEnd. 3 mouse endothelial cells, 2 citations

APC anti-mouse CD90.2: BioLegend, \#105312, clone 30-H12

RRID: AB_313183

Validated by the manufacturer by flow cytometry on C57BL/6 mouse thymocytes, 12 citations

FITC anti-mouse Ly-51 (6C3): BioLegend, \#108305, clone 6C3

RRID: AB_313362

Validated by the manufacturer by flow cytometry on C57BL/ 6 mouse bone marrow cells, 6 citations

PerCP-eFluor 710 anti-mouse CD200: eBioscience, \#46-5200-82, clone OX90

RRID: AB_10598213

Validated by the manufacturer by flow cytometry on 57BL/6 splenocytes, 1 citation

Biotin anti-mouse CD51: BD Pharmingen, \#551380, clone RMV-7

RRID: AB 394174

Validated by the manufacturer by flow cytometry on BALB/c bone marrow leukocytes, 8 citations

PE anti-Ki67: BD Pharmingen, \#556027, clone B56

RRID: AB 2266296

Validated by the manufacturer by flow cytometry on permeabilized MOLT-4 cells, 14 citations

PE anti-active Caspase 3: BD Pharmingen, \#550821, clone C92-605

RRID: AB 393906

Validated by the manufacturer by flow cytometry on camptothecin treated Jurkat cells, 3 citations

AlexaFluor 647 anti-SOX9: Cell Signaling Technology, \#71273, clone D8G8H

RRID: AB_2799799

Validated by the manufacturer by flow cytometry on HeLa cells (blue) and A-204 cells, 12 citations

APC anti-mouse CD146: BioLegend, \#134712, clone ME-9F1

RRID: AB_2563088

Validated by the manufacturer by flow cytometry on Mouse endothelial cells, 3 citations

Pacific Blue anti-mouse F4/80: BioLegend, \#123124, clone BM8

RRID: AB_893475

Validated by the manufacturer by flow cytometry on Thioglycolate-elicited Balb/c mouse peritoneal macrophages, 21 citations

PerCP/Cy5.5 anti-mouse CD31: BioLegend, \#102522, clone MEC13.3

RRID: AB 2566761

Validated by the manufacturer by flow cytometry on C57BL/6 mouse splenocytes, 11 citations

Primary antibodies for immunohistochemical analysis:

BrdU: Bio-Rad, \#OBT0030, clone BU 1/75-ICR1, Rat

RRID: AB 609568

Validated by the manufacturer for immunohistochemistry on formalin-fixed paraffin-embedded tissue, 31 citations

CD31: BD Biosciences, 550274, clone MEC 13.3, Rat

RRID: AB 393571

Validated by the manufacturer for immunohistochemistry on zinc-fixed paraffin-embedded section of U-87 MG tumor in mouse brain, 7 citations

type I collagen: Novus Biologicals, clone NB600-408, polyclonal, rabbit

RRID: AB 343276

Validated by the manufacturer for immunohistochemistry on FFPE sections of mouse pancreas tissue and rat colon tissue, 28 citations

type II collagen: Merck Millipore, MAB8887, monoclonal, mouse, clone 6B3

RRID: AB_2260779

Validated by the manufacturer for immunohistochemistry on fetal cartilage, 30 citations

SOX9: Novus Biologicals, NBP1-85551, polyclonal, rabbit

RRID: AB 11002706

Validated by the manufacturer for immunohistochemistry on FFPE sections of human colorectal cancer, glioma, skeletal muscle and small intestine, 4 citations 
CPT1a: Cell Signaling Technology, \#12252, clone D3B3, rabbit RRID: AB 2797857

Validated by the manufacturer on HeLa, PANC-1 and MCF7 cells, 16 citations

GLUT1: Cell Signaling Technology, \#12939, clone D3J3A, rabbit

RRID: AB_2687899

Validated by the manufacturer on HepG2 and Huh6 cells, 9 citations

FoxO3a: Cell Signaling Technology, \#2497, clone 75D8, rabbit

RRID: AB 836876

Validated by the manufacturer on SH-SY5Y cells IGF-I or LY294002 treated, 230 citations

Primary antibodies for immunocytochemical analysis:

FoxO1: Cell Signaling Technology, \#2880, clone C29H4, rabbit

RRID: AB_2106495

Validated by the manufacturer for immunofluorescent analysis in IGROV-1cells, 409 citations

FoxO3a: Cell Signaling Technology, \#2497, clone 75D8, rabbit

RRID: AB_836876

Validated by the manufacturer for immunofluorescent analysis on SH-SY5Y cells, 230 citations

Primary antibodies for Western Blot

SOX9: Novus Biologicals, NBP1-85551, polyclonal, rabbit

RRID: AB 11002706

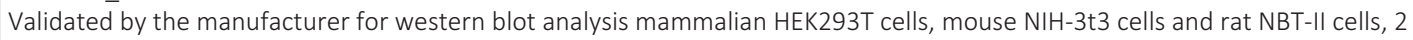
citations

FoxO1: Cell Signaling Technology, \#2880, clone $\mathrm{C29H4}$, rabbit

RRID: AB_2106495

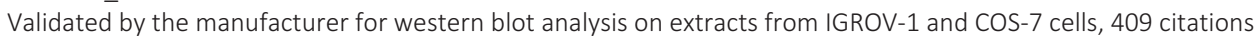

FoxO3a: Cell Signaling Technology, \#2497, clone 75D8, rabbit

RRID: AB_836876

Validated by the manufacturer for western blot analysis on extracts from Jurkat and PC3 cells, 230 citations

LC3B: Cell Signaling Technology, \#3868, clone D11, rabbit

RRID: AB 2137707

Validated by the manufacturer for western blot analysis on extracts of various cell line treated with chloroquine, 31 citations

$\beta$-actin: Sigma-Aldrich, \#A5441, clone AC-15, mouse

RRID: AB 476744

Validated by the manufacturer for western blot analysis on cultured human or chicken fibroblast cell extracts, 299 citations

Lamin A/C: Santa Cruz Biotechnology, \#sc-376248, clone E-1, mouse

RRID: AB_10991536

Validated by the manufacturer for western blot analysis on cell extracts of different mouse and human cell lines, 4 citations

Primary antibodies for ChIP-qPCR:

FoxO1: Abcam, \#ab39670, rabbit

RRID: AB_732421

Validated by the manufacturer for ChIP analysis on mouse T cells, 33 citations

FoxO3a: Abcam, \#ab12162, rabbit

RRID: AB_298893

Validated by the manufacturer for ChIP analysis on pig coronary artery endothelial cells, 30 citations

$\beta$-actin: Sigma-Aldrich, \#A5441, clone AC-15, mouse

RRID: AB 476744

Validated by the manufacturer for western blot analysis on cultured human or chicken fibroblast cell extracts, 299 citations

Lamin A/C: Santa Cruz Biotechnology, \#sc-376248, clone E-1, mouse

RRID: AB 10991536

Validated by the manufacturer for western blot analysis on cell extracts of different mouse and human cell lines, 4 citations

Primary antibodies for ChIP-qPCR:

FoxO1: Abcam, \#ab39670, rabbit

RRID: AB 732421

Validated by the manufacturer for ChIP analysis on mouse T cells, 33 citations

FoxO3a: Abcam, \#ab12162, rabbit 


\section{Eukaryotic cell lines}

Policy information about cell lines

Cell line source(s)

C3H10T1/2 cells were obtained from the RIKEN Cell Bank

Authentication

None of the cell lines used were authenticated

Mycoplasma contamination

Cell lines were routinely tested for mycoplasma contamination and found negative

Commonly misidentified lines

(See ICLAC register)

The C3H10T1/2 cell line is not among the commonly misidentified cell lines

\section{Animals and other organisms}

Policy information about studies involving animals; ARRIVE guidelines recommended for reporting animal research

Laboratory animals

Analysis was performed on 3-5 day-old mice or 8-10 week-old male and female mice. C57BL/6J mice (Janvier Labs), 129/Sv mice (Janvier Labs), B6.Cg-Tg(CAG-EGFP) mice (Hadjantonakis, A. K. et al., Mech. Dev., 1998), B6.Cg-Tg(Col1a1-cre/ERT2,-

DsRed)1Smkm/J mice (Ouyang, Z. et al., Bone, 2014), B6;129S4-Sox9tm1.1Tlu/J mice and B6.Cg-Tg(CAG-DsRed*MST)1Nagy/J mice (The Jackson Laboratory) were used in this study.

All colonies were housed and bred in individually ventilated cages in the animal facility of the KU Leuven.

Wild animals

The study did not use any wild animals

Field-collected samples

The study did not include field-collected samples

Ethics oversight

All animal experiments were conducted according to the regulations and with approval of the Animal Ethics Committee of the KU Leuven.

Note that full information on the approval of the study protocol must also be provided in the manuscript.

\section{Flow Cytometry}

\section{Plots}

\section{Confirm that:}

$\square$ The axis labels state the marker and fluorochrome used (e.g. CD4-FITC).

$\square$ The axis scales are clearly visible. Include numbers along axes only for bottom left plot of group (a 'group' is an analysis of identical markers).

$\bigotimes$ All plots are contour plots with outliers or pseudocolor plots.

$\bigotimes$ A numerical value for number of cells or percentage (with statistics) is provided.

\section{Methodology}

Sample preparation

Instrument

Software

Cell population abundance

Gating strategy
Isolation of mouse skeletal stem cells was adapted from a previously described protocol (Chan, C. K., et al., Cell, 2015). Long bones of 3-5 day old mice were dissected, muscle was cleared away and bones were minced using a scalpel. Bone fragments were then digested in $\alpha$-MEM supplemented with $3 \mathrm{mg} / \mathrm{ml}$ collagenase $I I, 4 \mathrm{mg} / \mathrm{ml}$ dispase (both from Gibco) and 100U/ml DNAse I (Sigma) at $37^{\circ} \mathrm{C}$ for 45 minutes, with replacement of the digest medium every 15 minutes. Cell suspensions were passed through a $70 \mu \mathrm{m}$ cell strainer, washed with PBS containing 2\% FBS and stained with antibodies against CD45, Ter119, Tie2, CD105, CD90.2, 6C3 (BioLegend), CD51 (BD Pharmingen) and CD200 (eBioscience), and with the viability dye 7aminoactinomycin $\mathrm{D}$ (BD Pharmingen).

For the isolation of skeletal muscle-derived cell populations, hindlimb skeletal muscles, including quadriceps, soleus, gastrocnemius and tibialis anterior, were dissected from 8-week old CAG-DsRed mice, minced using a scalpel and digested in $\alpha$ MEM medium supplemented with $3 \mathrm{mg} / \mathrm{ml}$ collagenase II, $4 \mathrm{mg} / \mathrm{ml}$ dispase and $100 \mathrm{U} / \mathrm{ml}$ DNAse I at $37^{\circ} \mathrm{C}$ for 60 minutes. Every 15 minutes samples were pipetted up and down vigorously using a $10 \mathrm{ml}$ serological pipette to break up tissue fragments. Cell

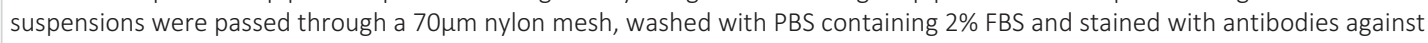
CD45, Ter119, CD31, F4/80 and CD146 (BioLegend), and with 7AAD (BD Pharmingen).

BD LSRII

BD FACSDiva

Post-sort purity was not determined

Phenotypic skeletal stem cells (7AAD-CD45-Ter119-Tie2-CD51+CD105-CD90.2-6C3-CD200+) were sorted on a BD FACSAria II (BD 
Immunophenotypically-defined macrophages (7AAD-CD45+F4/80+), endothelial cells (7AAD-CD45-Ter119-F4/80-CD31+CD146+) and pericytes (7AAD-CD45-Ter119-F4/80-CD31-CD146+) (Extended Data Fig. 9b) were sorted on a BD FACSAria II. Gating strategies are described in Extended Data Fig. 9b.

Analysis of SOX9high cells: Gating for SOX9high cells was set to have approximately $10 \%$ SOX9high cells in control conditions for all cell types.

$\triangle$ Tick this box to confirm that a figure exemplifying the gating strategy is provided in the Supplementary Information. 